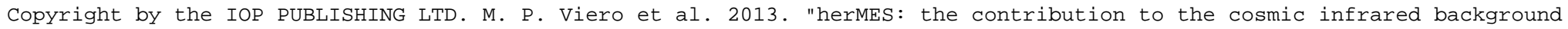
from galaxies selected by mass and redshift," ApJ 779 32 doi:10.1088/0004-637X/779/1/32

\title{
HerMES: THE CONTRIBUTION TO THE COSMIC INFRARED BACKGROUND FROM GALAXIES SELECTED BY MASS AND REDSHIFT*
}

\author{
M. P. Viero ${ }^{1}$, L. Moncelsi ${ }^{1}$, R. F. Quadri ${ }^{2,24}$, V. Arumugam ${ }^{3}$, R. J. AsseF ${ }^{4,23,25}$, M. Béthermin ${ }^{5,6}$, J. Bock $^{1,4}$, C. Bridge $^{1}$, \\ C. M. Casey ${ }^{7}$, A. Conley ${ }^{8}$, A. Cooray ${ }^{1,9}$, D. FArraH ${ }^{10}$, J. GlenN ${ }^{8,11}$, S. Heinis ${ }^{12}$, E. Ibar ${ }^{13,14}$, S. IKarashi ${ }^{15}$, R. J. Ivison ${ }^{3,14}$, \\ K. Kohno ${ }^{15}, 16$, G. Marsden ${ }^{17}$, S. J. Ollver ${ }^{18}$, I. G. Roseboom ${ }^{3}$, B. Schulz ${ }^{1,19}$, D. ScotT ${ }^{17}$, P. Serra ${ }^{6}$, M. VACCARI ${ }^{20}$, \\ J. D. VieIRA ${ }^{1}$, L. WANG ${ }^{21}$, J. WARDLOW ${ }^{9}$, G. W. Wilson ${ }^{22}$, M. S. Yun ${ }^{22}$, AND M. ZemCov ${ }^{1,4}$ \\ ${ }^{1}$ California Institute of Technology, 1200 East California Boulevard, Pasadena, CA 91125, USA; marco.viero@ caltech.edu \\ ${ }^{2}$ Carnegie Observatories, Pasadena, CA 91101, USA \\ ${ }^{3}$ Institute for Astronomy, University of Edinburgh, Royal Observatory, Blackford Hill, Edinburgh EH9 3HJ, UK \\ ${ }^{4}$ Jet Propulsion Laboratory, 4800 Oak Grove Drive, Pasadena, CA 91109, USA \\ ${ }^{5}$ Laboratoire AIM-Paris-Saclay, CEA/DSM/Irfu-CNRS-Université Paris Diderot, CE-Saclay, pt courrier 131, F-91191 Gif-sur-Yvette, France \\ ${ }^{6}$ Institut d'Astrophysique Spatiale (IAS), bâtiment 121, Université Paris-Sud 11 and CNRS (UMR 8617), F-91405 Orsay, France \\ ${ }^{7}$ Institute for Astronomy, University of Hawaii, 2680 Woodlawn Drive, Honolulu, HI 96822, USA \\ ${ }^{8}$ Center for Astrophysics and Space Astronomy 389-UCB, University of Colorado, Boulder, CO 80309, USA \\ ${ }^{9}$ Department of Physics and Astronomy, University of California, Irvine, CA 92697, USA \\ ${ }^{10}$ Department of Physics, Virginia Tech, Blacksburg, VA 24061, USA \\ ${ }^{11}$ Department of Astrophysical and Planetary Sciences, CASA 389-UCB, University of Colorado, Boulder, CO 80309, USA \\ ${ }^{12}$ Laboratoire d'Astrophysique de Marseille-LAM, Université d'Aix-Marseille and CNRS, UMR7326, \\ 38 rue F. Joliot-Curie, F-13388 Marseille Cedex 13, France
${ }^{13}$ Departamento de Astronomía y Astrofísica, Pontificia Universidad Catolica de Chile, Vicuña Mackenna 4860, Casilla 306, Santiago 22, Chile \\ ${ }^{14}$ UK Astronomy Technology Centre, Royal Observatory, Blackford Hill, Edinburgh EH9 3HJ, UK \\ ${ }^{15}$ Institute of Astronomy, University of Tokyo, 2-21-1 Osawa, Mitaka, Tokyo 181-0015, Japan \\ ${ }^{16}$ Research Center for the Early Universe, University of Tokyo, 7-3-1 Hongo, Bunkyo, Tokyo 113-0033, Japan \\ ${ }^{17}$ Department of Physics \& Astronomy, University of British Columbia, 6224 Agricultural Road, Vancouver, BC V6T 1Z1, Canada \\ ${ }^{18}$ Astronomy Centre, Department of Physics and Astronomy, University of Sussex, Brighton BN1 9QH, UK \\ ${ }^{19}$ Infrared Processing and Analysis Center, MS 100-22, California Institute of Technology, JPL, Pasadena, CA 91125, USA \\ ${ }^{20}$ Astrophysics Group, Physics Department, University of the Western Cape, Private Bag X17, 7535, Bellville, Cape Town, South Africa \\ ${ }^{21}$ Institute for Computational Cosmology, Department of Physics, University of Durham, South Road, Durham, DH1 3LE, UK \\ ${ }^{22}$ Department of Astronomy, University of Massachusetts, Amherst, MA 01003, USA \\ ${ }^{23}$ Núcleo de Astronomía de la Facultad de Ingeniería, Universidad Diego Portales, AV. Ejército Libertador 441, Santiago, Chile \\ Received 2013 April 1; accepted 2013 October 18; published 2013 November 22
}

\begin{abstract}
We quantify the fraction of the cosmic infrared background (CIB) that originates from galaxies identified in the UV/optical/near-infrared by stacking 81,250 $\left(\sim 35.7 \operatorname{arcmin}^{-2}\right) K$-selected sources $\left(K_{\mathrm{AB}}<24.0\right)$ split according to their rest-frame $U-V$ versus $V-J$ colors into 72,216 star-forming and 9034 quiescent galaxies, on maps from Spitzer/MIPS (24 $\mu \mathrm{m})$, Herschel/PACS (100, $160 \mu \mathrm{m})$, Herschel/SPIRE (250, 350, 500 $\mu \mathrm{m})$, and AzTEC $(1100 \mu \mathrm{m})$. The fraction of the CIB resolved by our catalog is $(69 \% \pm 15 \%)$ at $24 \mu \mathrm{m},(78 \% \pm 17 \%)$ at $70 \mu \mathrm{m}$, $(58 \% \pm 13 \%)$ at $100 \mu \mathrm{m},(78 \% \pm 18 \%)$ at $160 \mu \mathrm{m},(80 \% \pm 17 \%)$ at $250 \mu \mathrm{m},(69 \% \pm 14 \%)$ at $350 \mu \mathrm{m},(65 \% \pm 12 \%)$ at $500 \mu \mathrm{m}$, and $(45 \% \pm 8 \%)$ at $1100 \mu \mathrm{m}$. Of that total, about $95 \%$ originates from star-forming galaxies, while the remaining $5 \%$ is from apparently quiescent galaxies. The CIB at $\lambda \lesssim 200 \mu \mathrm{m}$ appears to be sourced predominantly from galaxies at $z \lesssim 1$, while at $\lambda \gtrsim 200 \mu \mathrm{m}$ the bulk originates from $1 \lesssim z \lesssim 2$. Galaxies with stellar masses $\log \left(M / M_{\odot}\right)=9.5-11$ are responsible for the majority of the CIB, with those in the $\log \left(M / M_{\odot}\right)=9.5-10$ bin contributing mostly at $\lambda<250 \mu \mathrm{m}$, and those in the $\log \left(M / M_{\odot}\right)=10-11$ bin dominating at $\lambda>350 \mu \mathrm{m}$. The contribution from galaxies in the $\log \left(M / M_{\odot}\right)=9.0-9.5$ (lowest) and $\log \left(M / M_{\odot}\right)=11.0-12.0$ (highest) stellarmass bins contribute the least - both of order 5\% - although the highest stellar-mass bin is a significant contributor to the luminosity density at $z \gtrsim 2$. The luminosities of the galaxies responsible for the CIB shifts from combinations of "normal" and luminous infrared galaxies (LIRGs) at $\lambda \lesssim 160 \mu \mathrm{m}$, to LIRGs at $160 \lesssim \lambda \lesssim 500 \mu \mathrm{m}$, to finally LIRGs and ultra-luminous infrared galaxies at $\lambda \gtrsim 500 \mu \mathrm{m}$. Stacking analyses were performed using SIMSTACK, a novel algorithm designed to account for possible biases in the stacked flux density due to clustering. It is made available to the public at www.astro.caltech.edu/ viero/viero_homepage/toolbox.html.
\end{abstract}

Key words: cosmology: observations - galaxies: evolution - infrared: galaxies - large-scale structure of universe - submillimeter: galaxies

Online-only material: color figures

\section{INTRODUCTION}

\footnotetext{
* Herschel is an ESA space observatory with science instruments provided by European-led Principal Investigator consortia and with important participation from NASA.

${ }^{24}$ Hubble Fellow.

${ }^{25}$ NASA Postdoctoral Program Fellow.
}

The cosmic infrared background (CIB), discovered in Far Infrared Absolute Spectrophotometer (FIRAS) data from the Cosmic Background Explorer (COBE; Puget et al. 1996; Fixsen et al. 1998), originates from thermal re-radiation of 


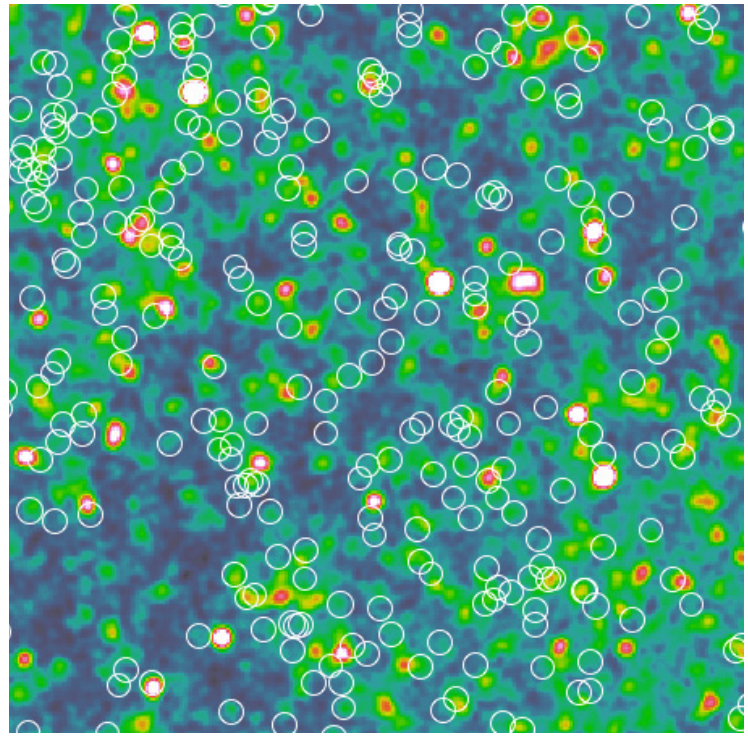

Figure 1. $0.25 \times 0.25$ SPIRE $250 \mu \mathrm{m}$ cutout of the UDS field, which has been smoothed and color-stretched to visually enhance the regions of submillimeter emission. Overlaid as white circles (diameters of $30^{\prime \prime}$ ) are the positions of starforming galaxies with masses between $\sim 10^{9.5-10.0} M_{\odot}$, in a single redshift slice spanning $z=1.0-1.5$. Note that very few, if any, of the $K$-selected sources are resolved in the SPIRE map, but that most lie on ridges of faint emission.

(A color version of this figure is available in the online journal.)

$\mathrm{UV} /$ optical starlight (and to a lesser extent active galactic nuclei, or AGN, emission) absorbed by dust grains. The total intensity of this background is roughly equal to that of the combined extragalactic UV, optical, and near-infrared backgrounds (the "cosmic optical background," or COB) indicating that, of all the light ever emitted by stars, about half has been absorbed and re-emitted by dust (Hauser \& Dwek 2001). While it is thought that the majority of the CIB originates from dusty star-forming galaxies (DSFGs; e.g., Le Floc'h et al. 2005; Lagache et al. 2005; Viero et al. 2009), how they relate to the sources that make up the $\mathrm{COB}$, and what fraction of the $\mathrm{CIB}$ is resolvable as optical sources, is still unclear.

To definitively answer that question, ideally the CIB would be resolved into individual sources and matched to optical counterparts, but from the first DSFGs imaged in the submillimeter (e.g., Smail et al. 1997; Barger et al. 1998; Hughes et al. 1998) it became quickly evident that identifying optical counterparts is a non-trivial exercise. The angular resolution afforded by singledish submillimeter observatories results in beams containing multiple sources, such that in deep observations the spatial variation of the sky intensity eventually reaches the so-called confusion limit (e.g., Nguyen et al. 2010). This situation is made worse by the strong evolution undergone by DSFGs between the present day and $z \sim 1$ (e.g., Pascale et al. 2009), such that only the brightest $\sim 1 \%$ of DSFGs (equivalent to $\sim 15 \%$ of the CIB; Oliver et al. 2010b) at $250 \mu \mathrm{m}$ is resolvable into point sources. This is illustrated in Figure 1, where a $0.25 \times 0.25 \mathrm{deg}^{2}$ cutout of the Spectral and Photometric Imaging REceiver (SPIRE) $250 \mu \mathrm{m}$ map is overlaid with positions of star-forming galaxies with masses between $\sim 10^{9.5-10.0} M_{\odot}$, at $z=1.0-1.5$. The map is smoothed and color-stretched to highlight the regions of emission. It is clear that very few of the sources are detected individually - the rest lie almost exclusively on ridges of faint emission.

Given ancillary data of sufficient quality, this limitation can be overcome by stacking. Conceptually, stacking is very simple: imagine cutting out hundreds of thumbnails from a map centered on the positions where galaxies are known to be, and averaging those thumbnails together until an image of the average galaxy emerges from the noise. These positional priors can come in many forms, e.g., they could be catalogs of UV, optical, IR, or radio sources. Note that the output is the average of that population in the stacked maps, i.e., there will likely be sources whose actual fluxes are higher or lower. Thus, the more homogeneous the sources comprising the input list, the more meaningful the stacked flux will be.

Stacking has been successfully applied to infrared maps by numerous groups looking to resolve the CIB with resolved sources. Frequently, Multi-band Imaging Photometer for Spitzer (MIPS; Rieke et al. 2004) $24 \mu \mathrm{m}$ sources were used as positional priors because bright $24 \mu \mathrm{m}$ sources are usually correlated with far-infrared (FIR) and submillimeter emission, and because of the large fraction of sources that are resolved in $24 \mu \mathrm{m}$ maps ( 70\%; Papovich et al. 2004). For example, Dole et al. (2006) showed that much of the CIB at 70 and $160 \mu \mathrm{m}$ is resolved by sources whose flux densities at $24 \mu \mathrm{m}$ are $S \gtrsim 60 \mu \mathrm{Jy}$. Similarly, by stacking on maps from the Balloonborne Large-Aperture Submillimeter Telescope (BLAST; Pascale et al. 2008), Devlin et al. (2009) and Marsden et al. (2009) demonstrated that close to the full intensity of the CIB at 250, 350 , and $500 \mu \mathrm{m}$ is resolved by $24 \mu \mathrm{m}$ sources, and that roughly half of the CIB at $500 \mu \mathrm{m}$ originates at $z>1.2$. Jauzac et al. (2011) estimated the contribution to the CIB brightness at 70 and $160 \mu \mathrm{m}$ from $24 \mu \mathrm{m}$ sources by stacking in narrow redshift bins spanning $0<z<1.05$. Berta et al. (2011), using Photodetector Array Camera and Spectrometer (PACS), and Béthermin et al. (2012c), using SPIRE maps, reconstructed number counts to flux densities below the confusion limit by stacking with $24 \mu \mathrm{m}$ priors, and found that the integral of their counts resolved $58 \%-74 \%$, and $55 \%-73 \%$ of the CIB at their respective wavelengths.

Stacking has been used to address other questions as well. Pascale et al. (2009), stacking $24 \mu \mathrm{m}$ sources on BLAST maps, measured the evolution of the infrared luminosity density with redshift, finding a significant rise in the temperatures and luminosities of sources with increasing redshift. Oliver et al. (2010a) used Spitzer "bandmerged" catalogs to measure the massdependency of specific star formation rates (sSFR) - the star formation rate (SFR) of the galaxy divided by its mass-over the redshift range $0<z<2$. Viero et al. (2012) stacked highredshift massive galaxies from the GOODS-NICMOS Survey (GNS; Conselice et al. 2011) on maps from PACS at 70-160 $\mu \mathrm{m}$ (Poglitsch et al. 2010), $870 \mu \mathrm{m}$ from LABOCA (Weiß et al. 2009), and BLAST, finding that the bulk of the star formation occurs in disk-like galaxies, with a hint that spheroid-like galaxies harbor a low level of star formation as well. Similarly, Hilton et al. (2012) stacked a stellar-mass selected sample of $1.5<z<3$ galaxies drawn from the GNS on SPIRE maps and found evidence for an increasing fraction of dust-obscured star formation with stellar mass. Heinis et al. (2013) stacked ultraviolet selected galaxies at $z \sim 1.5$ on SPIRE maps, finding that the mean infrared luminosity is correlated to the slope of the UV continuum, $\beta$. Penner et al. (2011) stacked $24 \mu \mathrm{m}$ sources in AzTEC $(1.1 \mathrm{~mm})$ and MAMBO $(1.2 \mathrm{~mm})$ data, finding much of the resolved background at those wavelengths originates from $z>1.3$.

While conceptually simple, in practice proper stacking is subtle and not without controversy. For sources that are uncorrelated (i.e., not clustered around other galaxies) the 
technique returns an unbiased estimate of the average flux density (e.g., Marsden et al. 2009; Viero et al. 2012). But if sources are clustered-which they inevitably will be-then a bias at some level will be present and must be accounted for.

Many solutions have been proposed to address this problem: some, like Béthermin et al. (2012c) correct for boosting with simulations. Alternatively, Béthermin et al. (2012c) and Heinis et al. (2013) fit the measured correlation function to the excess width of the measured stacked beam to estimate a correction. Bourne et al. (2011) use a median statistic to perform their stacking, which is shown to be resistant to biases induced by outliers.

Still another method, developed independently by Kurczynski \& Gawiser (2010), Roseboom et al. (2010), and Bourne et al. (2012), fits for the flux densities of multiple (correlated) lists simultaneously, thereby accounting for correlations as a part of the stack. The advantage of this technique is that it makes few assumptions and naturally takes into account the possibility that the potential clustering bias may be redshift and luminosity dependent. Here we build upon this technique to simultaneously measure the mean flux densities of galaxies selected by mass and divided into mass and redshift bins.

Our goal is to gain a better understanding of the contribution to the CIB from galaxies identified in the optical and near-infrared. In Section 2 we present the data, and in Section 3.1 we present our method, demonstrating its effectiveness with simulations in Section 3.3. We ultimately use it to determine the total contribution to the CIB from $K$-selected galaxies (Section 4.3), and its dependence on redshift (Section 4.4), mass and color (Section 4.5), and luminosity (Section 4.6). The dependence of sSFR on these variables will be explored in a forthcoming paper (V. Arumugam et al., in preparation). When required, we assume a Chabrier (2003) initial mass function (IMF) and a flat $\Lambda$ CDM cosmology with $\Omega_{\mathrm{M}}=0.274, \Omega_{\Lambda}=0.726$, $H_{0}=70.5 \mathrm{~km} \mathrm{~s}^{-1} \mathrm{Mpc}^{-1}$, and $\sigma_{8}=0.81$ (Komatsu et al. 2011).

\section{DATA}

We perform our analysis on the UKIRT Infrared Deep Sky Survey (UKIDSS; Lawrence et al. 2007), Ultra-Deep Survey (UDS) field, centered at coordinates $2^{\mathrm{h}} 17^{\mathrm{m}} 50^{\mathrm{s}},-5^{\circ} 6^{\prime} 0^{\prime \prime}$. The UDS is the deepest survey undertaken by UKIDSS, covering $0.8 \mathrm{deg}^{2}$ in $J, H$, and $K$ to nominal $5 \sigma$ depths of 26.9, 25.9, 24.9 mag $[\mathrm{AB}]$. Catalogs are based on optical and near-infrared (NIR) data in this field, while the maps on which the stacking analyses are performed span the mid-infrared to submillimeter. Here we briefly describe the catalog and maps.

\subsection{Optical/Near-infrared Catalog}

Galaxy positions and redshifts come from a catalog based on the UKIDSS Ultra-Deep Survey (UDS; Lawrence et al. 2007; Warren et al. 2007) Data Release 8 and supplementary data (R. J. Williams et al. 2013, in preparation). Source detection, photometry, and spectral energy distribution (SED) fitting are described in detail by Williams et al. (2009), with recent updates to the catalog discussed by Quadri et al. (2012). A brief summary of the data follows: sources are detected in the UDS $K$-band mosaic using Source Extractor v2.5.0 (Bertin \& Arnouts 1996), and fluxes measured in other bands from point-spread function (PSF) matched images: $u^{\prime}$ from archival Canada-France-Hawaii Telescope data, $B V R i^{\prime} z^{\prime}$ from the Subaru-XMM Deep Survey (Furusawa et al. 2008), JHK from the UDS DR8, and Spizter/IRAC imaging at 3.6, 4.5, 5.8,

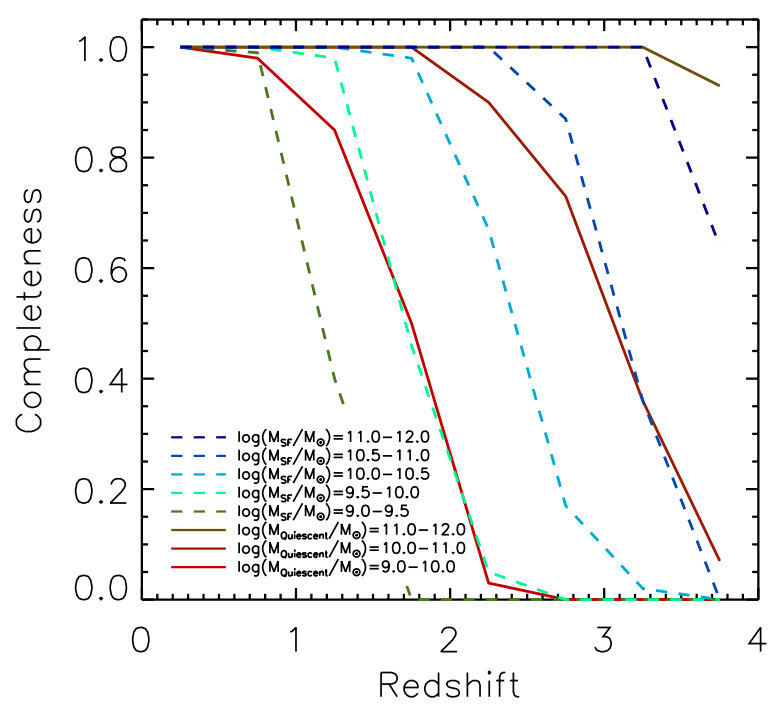

Figure 2. Completeness estimates plotted vs. redshift for star-forming and quiescent galaxies in bins of stellar mass.

(A color version of this figure is available in the online journal.)

and $8 \mu \mathrm{m}$ in the UDS field. An inspection of the number counts suggests that our catalog is essentially complete to $K_{\mathrm{AB}}<24.0$ for non-stellar objects, although it is difficult to rule out the possibility that there are a small number of diffuse and extended sources that do not make it into our sample. The de-blending technique of Labbé et al. (2006) and Wuyts et al. (2007) is used to extract matched IRAC fluxes. Objects near bad pixels in the optical or near-infrared images, or those with no optical coverage, are excluded, as well as stars, saturated sources, severe blends, or those near the edge of the image. These quality checks reduce the catalog from 171,392 to 81,250 objects, and the resulting effective image area is $\sim 0.63 \mathrm{deg}^{2}$.

Photometric redshifts and rest-frame colors are derived by fitting the multi-band photometry with EAZY (Brammer et al. 2008). Stellar masses are obtained with FAST (Kriek et al. 2009) using a Chabrier (2003) IMF, solar metallicity, and Bruzual \& Charlot (2003) stellar population models.

Quiescent and star-forming galaxies are classified based on the observed bimodality in a rest-frame color-color $U-V$ versus $V-J$ (hereafter $U V J$; Williams et al. 2009); this technique robustly separates red, dusty starbursts from red, dust-free, old stellar populations (see also Labbé et al. 2005; Wuyts et al. 2007). In addition to the quality checks listed above, sources whose best-fit SED has a high $\chi^{2}$ - which may be the result of poor photometry, artifacts in the images, or of a strong AGN component that is not a part of the EAZY template library- are excluded from the parent sample. Approximately 2,700 sources $(\sim 3 \%)$ exceed this limit; we explore the effect that inclusion of this subsample has on the total resolved CIB in Section 5.3.

Our sample is selected at $K_{\mathrm{AB}} \leqslant 24$, where the catalog is essentially $100 \%$ complete. We calculate the corresponding mass completeness values in a manner similar to Quadri et al. (2012). Briefly, we scale the fluxes and masses of galaxies at slightly brighter magnitudes down to $K_{\mathrm{AB}}=24$, and estimate the completeness as a function of mass and redshift as the fraction of objects below that mass at that redshift. Completeness estimates are plotted in Figure 2.

\subsection{Spitzer/MIPS}

We use publicly available Spitzer/MIPS maps at 70, and $160 \mu \mathrm{m}$ from the Spitzer Wide-Area Infrared Extragalactic 
Table 1

Nominal and Effective Beam Properties

\begin{tabular}{lccc}
\hline $\begin{array}{l}\text { Band } \\
(\mu \mathrm{m})\end{array}$ & $\begin{array}{c}\text { FWHM }_{\text {nom }} \\
(\operatorname{arcsec})\end{array}$ & $\begin{array}{c}\text { FWHM }_{\text {eff }} \\
(\operatorname{arcsec})\end{array}$ & $\begin{array}{c}\text { Area }_{\text {eff }} \\
\text { (steradians })\end{array}$ \\
\hline 24 & 6.0 & 6.3 & $1.548 \times 10^{-9}$ \\
70 & 18.0 & 19.3 & $1.296 \times 10^{-8}$ \\
100 & 7.4 & 7.0 & $1.305 \times 10^{-9}$ \\
160 & 11.3 & 11.2 & $3.341 \times 10^{-9}$ \\
250 & 18.1 & 17.6 & $0.994 \times 10^{-8}$ \\
350 & 25.2 & 23.9 & $1.765 \times 10^{-8}$ \\
500 & 36.6 & 35.2 & $3.730 \times 10^{-8}$ \\
1100 & 30.0 & 30.0 & $3.179 \times 10^{-8}$ \\
\hline
\end{tabular}

survey (Lonsdale et al. 2003) in the XMM Large-Scale Structure field (Surace 2005); and at $24 \mu \mathrm{m}$ from the Spitzer UDS survey (PI: J. Dunlop), DR2. Maps have rs levels of $0.5 \mathrm{mJy}, 1.8 \mathrm{mJy}$ and $19.9 \mu \mathrm{Jy}$, respectively. We note that the absolute calibration uncertainties are $4 \%, 7 \%$, and $12 \%$ at 24,70 , and $160 \mu \mathrm{m}$, respectively (Engelbracht et al. 2007; Gordon et al. 2007; Stansberry et al. 2007).

Following Béthermin et al. (2010), calibration corrections of $1.0509,1.10$, and 0.98 , and aperture corrections of 1.19, 1.21, and 1.20, are applied at 24, 70, and $160 \mu \mathrm{m}$. Maps are in native units of $\mathrm{MJy} \mathrm{sr}^{-1}$ (surface brightness), and are converted to $\mathrm{Jy} \mathrm{beam}^{-1}$ for this analysis by dividing the maps by the solid angles of the measured instrumental point response functions ${ }^{26}$ (PRFs), where $\Omega_{\text {beam }}=f / I_{0}=\int \mathrm{PRF} \mathrm{d} \Omega / \mathrm{PRF}_{0}$, and $\mathrm{PRF}_{0}$ is the peak value.

Also measured from the PRF is the effective FWHM of the best-fit Gaussian, which can differ from nominal by as much as $\sim 6 \%$. This is done by simply finding the two-dimensional Gaussian which provides the minimum value when differenced with the PRF, within a radius of $1.25 \times$ FWHM (chosen as the approximate minimum of the primary lobe). Effective area and FWHM for each band are listed in Table 1.

\subsection{Herschel/HerMES}

We use submillimeter maps at 100, 160, 250, 350, and $500 \mu \mathrm{m}$ from the Herschel Multi-tiered Extragalactic Survey (HerMES; Oliver et al. 2012). HerMES is a guaranteed time key project, and consists of maps of many of the well studied extragalactic fields, which are divided into tiers of depth and area, observed with both the SPIRE (Griffin et al. 2010) and the PACS (Poglitsch et al. 2010). The UDS is a level 4 field, consisting of 20 repeat observations, and will be made available via HEDAM ${ }^{27}$ (Roehlly et al. 2011), as part of DR2.

\subsubsection{Herschel/PACS}

Data at 100 and $160 \mu \mathrm{m}$ are taken with the PACS instrument and are processed with the Herschel Data Processing System (HIPE v10.2747; Ott 2010). Maps were made with UNIHIPE ${ }^{28}$ in combination with UNIMAP ${ }^{29}$ (Traficante et al. 2011; Piazzo 2013). Maps are made using the default parameters, with the exception of the image pixel sizes, which we set to 2 and 3 arcsec at 100 and $160 \mu \mathrm{m}$, respectively. The advantage of this

\footnotetext{
26 http://irsa.ipac.caltech.edu/data/SPITZER/docs/mips/

27 http://hedam.oamp.fr/HerMES/

28 herschel.asdc.asi.it/index.php?page=unimap.html

29 w3.uniroma1.it/unimap
}

mapmaker over the standard one available through HIPE is that it does not require strong high-pass filtering or masking of bright sources (e.g., Wieprecht et al. 2009) to produce reliable maps, thus avoiding the attenuation of the fainter population as was found in (e.g., Lutz et al. 2011; Viero et al. 2012). The rms depths of the maps are 0.44 and $1.5 \mathrm{mJy}$ at 100 and $160 \mu \mathrm{m}$, respectively.

\subsubsection{Herschel/SPIRE}

Data at 250, 350, and $500 \mu \mathrm{m}$ are observed with the SPIRE instrument to a depth of $11.2,9.3,13.4 \mathrm{mJy}(5 \sigma)$, not including confusion noise, which from Nguyen et al. (2010) is 24.0, 27.5 , and $30.5 \mathrm{mJy}(5 \sigma)$ at 250,350 and $500 \mu \mathrm{m}$, respectively. Absolute calibration is detailed in Swinyard et al. (2010), with calibration uncertainties of $\sim 7 \%$. Maps are made with 3 arcsec pixels using SMAP (Levenson et al. 2010; Viero et al. 2013b).

\subsection{AzTEC}

We use maps at $1100 \mu \mathrm{m}$ observed with the AzTEC camera (Wilson et al. 2008; Glenn et al. 1998) mounted on ASTE (Ezawa et al. 2004, 2008). The FWHM of the AzTEC beam on ASTE is $30 \operatorname{arcsec}$ at $1100 \mu \mathrm{m}$, and the field of view of the array is roughly circular with a diameter of 8 arcmin. Calibration errors are quoted for individual observations to be $6 \%-13 \%$ (Wilson et al. 2008; Austermann et al. 2010), depending on the source; here we adopt a value of $10 \%$. The area covered is smaller than at the other bands, totaling $\sim 0.32 \mathrm{deg}^{2}$ after cropping the noisy outer edge. These data will be presented by $\mathrm{S}$. Ikarashi et al. (in preparation).

\subsection{Color Corrections}

We apply color corrections to convert from the standard calibration to the actual measured SED of the stacked sources. As the part of the spectrum observed depends on the source's redshift, the color correction is applied after first finding the best-fit SED in each bin. Consequently, each color correction is unique, though the difference in any one band across the full redshift range is never greater than $\sim 10 \%$. The color corrections per band, from lowest to highest redshift, are: 0.99-1.02 (24 $\mu \mathrm{m})$; 0.97-1.02 (70 $\mu \mathrm{m}) ; 0.93-1.02(100 \mu \mathrm{m})$; 0.99-1.00 (160 $\mu \mathrm{m}) ; 0.98-0.99(250 \mu \mathrm{m}) ; 0.99-1.00(350 \mu \mathrm{m})$; 0.99-1.07 (500 $\mu \mathrm{m})$; and 0.96-0.99 (1100 $\mu \mathrm{m})$.

\section{METHOD FOR UNBIASED STACKING}

As was shown by Marsden et al. (2009), stacking is formally the covariance between a catalog (or multiple catalogs) of positions $C_{\alpha}$, containing $N_{\alpha}^{j}$ sources in pixel $j$, and a map, $M$. The mean of $N_{\alpha}^{j}$ is $\mu_{\alpha}$, which represents the average number of sources in catalog $\alpha$ per pixel. In the limit that sources are Poisson distributed on the scale of the beam, then the covariance is simply the mean of the map at positions $C_{\alpha}$, so that the average flux density of a given catalog is

$$
\hat{S}_{\alpha}=\frac{1}{N_{\text {pix }} \mu_{\alpha}} \sum_{j} M_{j} N_{\alpha}^{j},
$$

where $N_{\text {pix }}$ is the total number of pixels in the map. 
If the catalog (or catalogs) in question is correlated on the scale of the beam, $\mu$ can simply be replaced with the variance, $\sigma^{2}$. What this does not account for-as pointed out by e.g., Chary \& Pope (2010), Serjeant et al. (2010), and Kurczynski \& Gawiser (2010) - is the possibility that some other, fainter, and potentially numerous sources (or the sources in companion catalogs), may be correlated with the sources in that catalog, and that neglecting them could introduce a bias.

We now present an algorithm, whose formalism is similar to those of Kurczynski \& Gawiser (2010) and Roseboom et al. (2010), with the difference that only samples which could potentially be correlated (i.e., those in the same redshift range) are simultaneously fit. In the following section we provide the formalism, while step-by-step instructions are given in Section 3.2.

\subsection{Stacking Formalism}

The following is a generalization of the formalism presented in Marsden et al. (2009), and is applicable to any catalog, including those that are clustered at angular scales comparable to that of the beam. For a map, $M_{j}$, with pixels $j$, and a set of lists, $S_{\alpha}$ :

$$
\begin{aligned}
M_{j} & =n_{j}+\sum_{\alpha} S_{\alpha}\left(N_{\alpha}^{j}-\mu_{\alpha}\right) \\
& =n_{j}+S_{1}\left(N_{1}^{j}-\mu_{1}\right)+\ldots+S_{n}\left(N_{n}^{j}-\mu_{n}\right),
\end{aligned}
$$

where the $S_{\alpha}$ form the complete set of all objects in the universe.

Note that, unlike in Marsden et al. (2009), we need not assume that $N_{\alpha}^{j}$ be a Poisson-distributed number. Furthermore, separate lists can also be correlated, so that the covariances between them need not be non-zero. However, we still require that the instrumental noise is well behaved, i.e., $\left\langle n_{j}\right\rangle=0$, so that terms in $N_{\alpha}^{j} n_{j}$ vanish in the sum.

The amplitudes $S_{\alpha}$ and $N_{\alpha}^{j}$ in Equation (2) that satisfy $M_{j}$ can be quantified by writing their covariances with the map itself:

$$
\begin{aligned}
\operatorname{Cov}\left(M, N_{\alpha}\right) & =\frac{1}{N_{\mathrm{pix}}} \sum_{j} M_{j} N_{\alpha}^{j} \\
& =\frac{1}{N_{\mathrm{pix}}} \sum_{j} N_{\alpha}^{j} \sum_{\alpha} S_{\alpha}\left(N_{\alpha}^{j}-\mu_{\alpha}\right) \\
& =\frac{S_{\alpha}}{N_{\mathrm{pix}}}\left[\sum_{j}\left(N_{\alpha}^{j}\right)^{2}-\mu_{\alpha} \sum_{j} N_{\alpha}^{j}\right] \\
& +\sum_{\alpha^{\prime} \neq \alpha} \frac{S_{\alpha^{\prime}}}{N_{\mathrm{pix}}}\left[\sum_{j} N_{\alpha}^{j} N_{\alpha^{\prime}}^{j}-\mu_{\alpha^{\prime}} \sum_{j} N_{\alpha}^{j}\right],
\end{aligned}
$$

which can be rewritten in matrix form by defining amplitude and covariance vectors:

$$
\mathbf{S}=\left(\begin{array}{c}
S_{1} \\
S_{2} \\
\vdots \\
S_{n}
\end{array}\right) ; \operatorname{Cov}\left(M, N_{\alpha}\right)=\left(\begin{array}{c}
\operatorname{Cov}\left(M, N_{1}\right) \\
\operatorname{Cov}\left(M, N_{2}\right) \\
\vdots \\
\operatorname{Cov}\left(M, N_{n}\right)
\end{array}\right)
$$

and

$$
\begin{aligned}
& \left(\begin{array}{c}
\operatorname{Cov}\left(M, N_{1}\right) \\
\vdots \\
\operatorname{Cov}\left(M, N_{n}\right)
\end{array}\right)=\frac{1}{N_{\text {pix }}} \\
& \times\left(\begin{array}{ccc}
\sum_{j} N_{1}^{j}\left(N_{1}^{j}-\mu_{1}\right) & \cdots & \sum_{j} N_{1}^{j}\left(N_{n}^{j}-\mu_{n}\right) \\
\vdots & \ddots & \vdots \\
\sum_{j} N_{n}^{j}\left(N_{1}^{j}-\mu_{1}\right) & \cdots & \sum_{j} N_{n}^{j}\left(N_{n}^{j}-\mu_{n}\right)
\end{array}\right)\left(\begin{array}{c}
S_{1} \\
\vdots \\
S_{n}
\end{array}\right) .
\end{aligned}
$$

From the covariances and the $n \times n$ matrix which we label $\mathbf{A}$, $\mathbf{S}$ is then simply

$$
\tilde{\mathbf{S}}=\mathbf{A}^{-1} \operatorname{Cov}\left(M, N_{\alpha}\right) .
$$

Notice the resemblance that the linear system in Equation (5) bears to that of a least-squares fit

$$
y=\sum_{\alpha} a_{\alpha} x_{\alpha}=a_{1} x_{1}+a_{2} x_{2}+\ldots+a_{n} x_{n},
$$

whose residual is given by

$$
R^{2}=\sum_{j}\left[y_{j}-\left(a_{1} x_{1}^{j}+a_{2} x_{2}^{j}+\ldots+a_{n} x_{n}^{j}\right)\right]^{2} .
$$

In order to minimize this residual, we impose the following set of conditions:

$$
\begin{aligned}
& \frac{\partial R^{2}}{\partial a_{1}}=-2 \sum_{j}\left[y_{j}-\left(a_{1} x_{1}^{j}+a_{2} x_{2}^{j}+\ldots+a_{n} x_{n}^{j}\right)\right] x_{1}^{j}=0 ; \\
& \frac{\partial R^{2}}{\partial a_{2}}=-2 \sum_{j}\left[y_{j}-\left(a_{1} x_{1}^{j}+a_{2} x_{2}^{j}+\ldots+a_{n} x_{n}^{j}\right)\right] x_{2}^{j}=0 ; \\
& \frac{\partial R^{2}}{\partial a_{n}}=-2 \sum_{j}\left[y_{j}-\left(a_{1} x_{1}^{j}+a_{2} x_{2}^{j}+\ldots+a_{n} x_{n}^{j}\right)\right] x_{n}^{j}=0 .
\end{aligned}
$$

These can be expressed in matrix form as

$$
\begin{aligned}
& \left(\begin{array}{c}
\sum_{j} x_{1}^{j} y_{j} \\
\sum_{j} x_{2}^{j} y_{j} \\
\vdots \\
\sum_{j} x_{n}^{j} y_{j}
\end{array}\right)=\left(\begin{array}{cccc}
\sum_{j}\left(x_{1}^{j}\right)^{2} & \sum_{j} x_{1}^{j} x_{2}^{j} & \cdots & \sum_{j} x_{1}^{j} x_{n}^{j} \\
\sum_{j} x_{1}^{j} x_{2}^{j} & \sum_{j}\left(x_{2}^{j}\right)^{2} & \cdots & \sum_{j} x_{2}^{j} x_{n}^{j} \\
\vdots & \vdots & \ddots & \vdots \\
\sum_{j} x_{1}^{j} x_{n}^{j} & \sum_{j} x_{2}^{j} x_{n}^{j} & \cdots & \sum_{j}\left(x_{n}^{j}\right)^{2}
\end{array}\right) \\
& \quad \times\left(\begin{array}{c}
a_{1} \\
a_{2} \\
\vdots \\
a_{n}
\end{array}\right) .
\end{aligned}
$$

By comparing Equations (5) and (10), it is clear that the $a_{\alpha}$ vector maps into $S_{\alpha}$, the $y_{j}$ vector maps into $M_{j}$, the $\sum_{j} x_{\alpha}^{j} y_{j}$ 


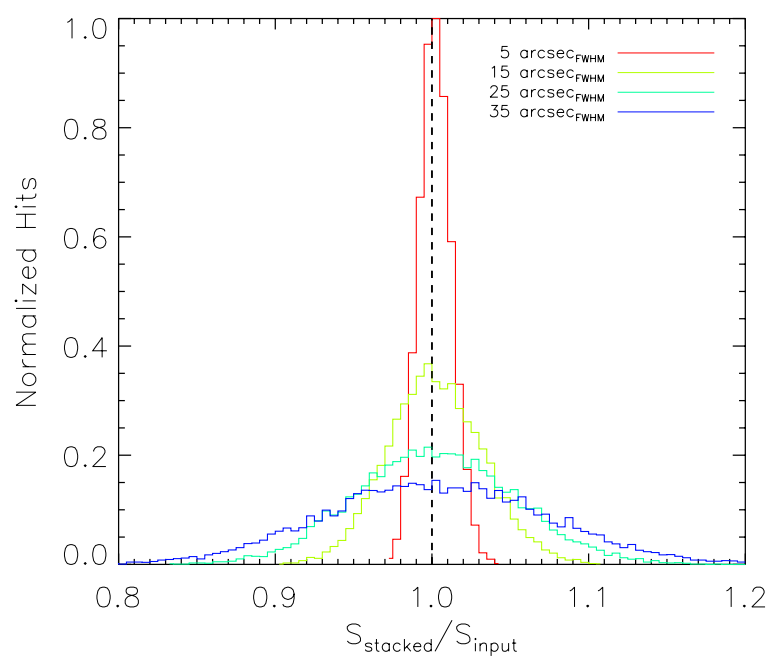

Figure 3. Test of the traditional stacking estimator on simulated maps with randomly distributed (i.e., unclustered) sources. Histograms show the resulting output vs. input flux densities of 10,000 iterations per beam size, for beams ranging from FWHM $=15^{\prime \prime}-35^{\prime \prime}$, and a source density of $\sim 2 \operatorname{arcmin}^{-2}$. The vertical dashed line at unity represents an unbiased estimate. For all beam sizes, the estimator is shown to be unbiased, though the errors increase with an increased number of sources per beam (i.e., for larger beams).

(A color version of this figure is available in the online journal.)

vector maps into the covariances $\operatorname{Cov}\left(M_{j}, N_{\alpha}^{j}\right)$, and the $x_{\alpha}^{j}$ vector maps into the $N_{\alpha}^{j}$ (which is mean-subtracted).

Therefore, solving Equation (5) for $S_{\alpha}$ is equivalent to finding the coefficients $a_{\alpha}$ in Equation (7) via a minimization routine. Specifically, the functional form can be operatively implemented using known quantities:

$$
M=\sum_{\alpha} S_{\alpha} C_{\alpha}=S_{1} C_{1}+S_{2} C_{2}+\ldots+S_{n} C_{n}
$$

where we define the $C_{\alpha}^{j}$ as a beam-convolved and meansubtracted version of the $N_{\alpha}^{j}$.

\subsection{Method in Practice}

Here we present the simultaneous stacking algorithm (SIMSTACK) used in this analysis, which we also make publicly available through an IDL code. ${ }^{30}$ The simultaneous stack is performed on one map at a time, and one group at a time, where groups are defined as catalogs which could potentially be correlated. For example, we group all lists in the same redshift range together (for a total of eight groups), as we expect galaxies of different masses but equal redshifts to be correlated with each other. In other words, we assume that galaxies in different redshift slices are uncorrelated, and can be dealt with independently. Then, regardless of the code used, the method can be broken into four simple steps:

Prepare $N$ lists of R.A. and decl. by group, e.g., we divide each group (redshift slice) into 8 lists of mass and $U V J$ color; thus $N=8$.

Construct $N$ layers, or "hits" maps, one for each list, where each pixel in the hits map contains the integer number of sources which falls into it.

\footnotetext{
30 www.astro.caltech.edu/ viero/viero_homepage/toolbox.html
}

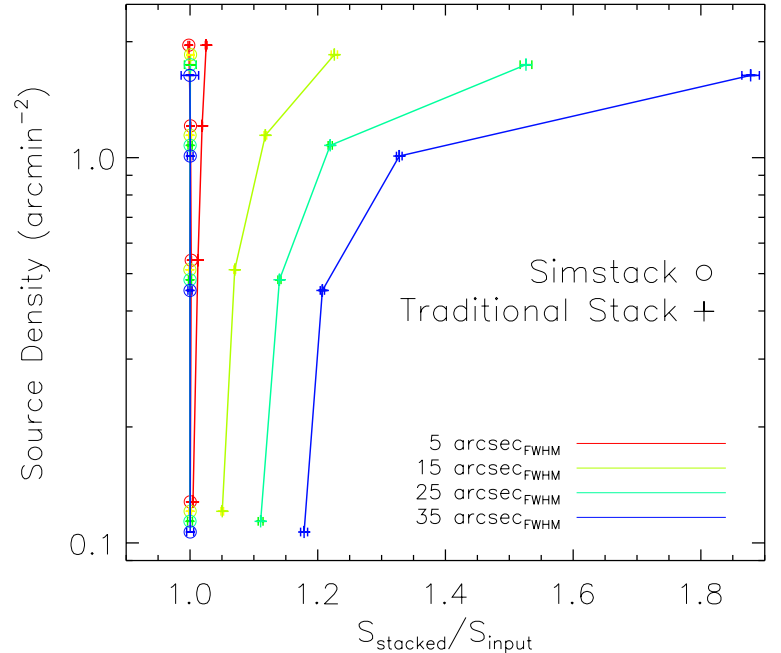

Figure 4. Test of the traditional and simultaneous stacking estimators on 10,000 clustered simulated maps. Recovered vs. input fluxes are measured as a function of source density and beam size. The traditional estimator performs well for beams smaller than 5 arcsec, but quickly becomes biased for bigger beams, particularly at higher source densities. The simultaneous stacking algorithm, SIMSTACK, on the other hand returns an unbiased estimate in all cases.

(A color version of this figure is available in the online journal.)

Convolve the $N$ layers with an effective PSF (we use a Gaussian) whose FWHM is equal to that of the effective instrumental beam of the real map. ${ }^{31}$

Regress the $N$ convolved layers with the real map of the sky, ideally weighted by the noise. ${ }^{32}$

Stacking should be performed on maps in Jy beam ${ }^{-1}$. Errors can be estimated with a bootstrap technique, as described in Section 3.4. Systematic errors in the method include beam area and calibration uncertainties. Note that calibration errors may be correlated between bands of the same instrument-an effect that should be accounted for when fitting models to stacked flux densities.

\subsection{Testing the Method}

Monte Carlo simulations consisting of 10,000 iterations are performed to test the estimator for biases. Two sets of simulated maps - one containing Poisson distributed (random) sources and the other of realistically correlated (clustered) sources - are constructed. Each map is a superposition of sources of varying mass, with the number of sources in each mass bin the same as that of real data. The flux densities given to the sources are drawn from Gaussian distributions centered on the flux densities of the measured mean stacked flux densities, and the width of the Gaussian five times that of the uncertainty on the stacked values, in order to introduce a significant level of stochasticity.

Sources in clustered simulated maps have their positions drawn from the actual positions of the catalog sources, in order to properly reproduce higher order correlations. Each map is then convolved with a Gaussian kernal approximating the instrumental beams, with FWHM values ranging from 5 to 35 arcsec.

\footnotetext{
31 If using the actual PRF of the instrument, take care that the orientation is correct, and if the field has been viewed at multiple angles, that the effective PRF is used.

32 As dictated by the formalism of Section 3.1, take care that the mean of the
} pixels to be fit in each layer equals zero. 


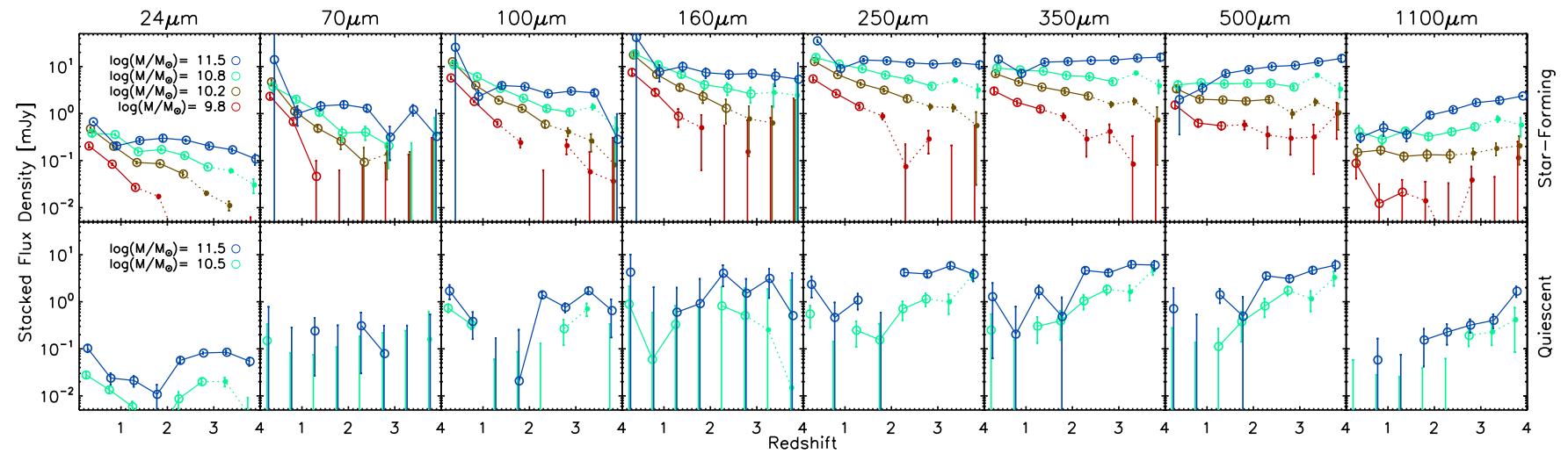

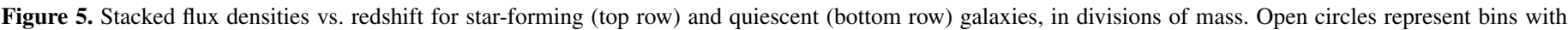

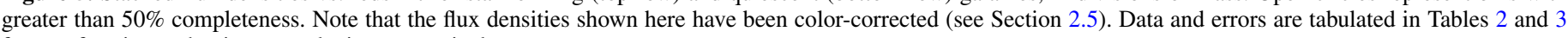
for star-forming and quiescent galaxies, respectively.

(A color version of this figure is available in the online journal.)

We then perform stacking analyses on the maps in two ways: (1) with a traditional (e.g., Marsden et al. 2009) estimator, and (2) with the SIMSTACK algorithm. Finally, the stacked flux densities are compared to the known input mean values. The histogram in Figure 3 illustrates how the traditional estimator returns an unbiased estimate of the mean flux $\left(S_{\text {stacked }} / S_{\text {input }} \approx 1\right)$ of an unclustered simulation, but with an uncertainty that depends on the beam size (see also Viero et al. 2012). Similarly, Figure 4 shows the bias versus source density (in number of sources per square arcmin) for clustered simulated maps, where the traditional estimator is represented by crosses and our simultaneous stacking method by circles. The data points are offset vertically for visual clarity. The traditional stacking estimator is relatively faithful for beams of FWHM $\leqslant 5^{\prime \prime}$, but quickly becomes biased, especially in catalogs with many sources. The simultaneous stacking instead returns an unbiased estimate of the mean flux density, with errors $\sigma=1 \%-3 \%$, increasing with increasing beam size.

\subsection{Estimating Uncertainties}

In addition to measurement errors, we must account for potential systematic errors introduced by photometric redshifts. To address this potential bias we developed an extension of the typical bootstrap technique, hereafter referred to as the extended bootstrap technique, or EBT. Like a typical bootstrap, the EBT assembles new bins for stacking from the sources in the parent catalog, with the difference that rather than simply drawing sources randomly from the original bin, all of the sources in the catalog are first perturbed according to their redshift uncertainties, and then new bins are assembled from the new redshifts and masses. Simulated redshifts are determined by drawing randomly from the redshift probability distribution output by the photometric redshift code, EASY. Simulated masses, which change depending on redshift of the source, must be estimated as well. However, as estimating a new mass for every new redshift for every source would be overly labor intensive-particularly considering that most perturbations from the nominal redshift are rather small-we instead use the fact that the mass is a strong function of $K$-band magnitude with some slight additional dependence on $J-K$ color, and we estimate the new mass using the perturbed redshift and the observed magnitude and color. Finally, each simulated catalog is split up into bins resembling those of the original stack and new stacked flux densities are estimated with SIMSTACK. This is done 1000 times. Measurement and bootstrap errors are then added in quadrature, though we note that the error budget is dominated by the EBT estimates.

The EBT accounts for the possibility of cross-contamination of galaxies across redshift and mass bins, in addition to the stochasticity of the catalog members measured by the traditional bootstrap technique, thus resulting in a more realistic error. We find that the EBT increases uncertainties by an average of 22\%; where the correction in bins with better photometry is less; while the correction in bins with poor photometry (i.e., high redshift and/or low mass) can be as much as $50 \%$. Note that these uncertainties account for both instrumental and confusion noise, as well as for any pixel-pixel correlations that map-making may introduce.

Last, systematic errors arising from estimating the solid angles (or beam areas) of the MIPS PSFs (Béthermin et al. 2010), as well as calibration uncertainties at all wavelengths (Engelbracht et al. 2007; Gordon et al. 2007; Stansberry et al. 2007; Swinyard et al. 2010), must be taken into account, particularly when estimating the contribution to the CIB from galaxies. These errors are accounted for empirically through inclusion into the Monte Carlo simulation used to estimate the ultimate errors.

\section{RESULTS}

\subsection{Stacked Flux Densities}

Stacked flux densities and $1 \sigma$ uncertainties are shown for starforming and quiescent galaxies in the top and bottom panels of Figure 5, and listed in Tables 2 and 3, respectively. We find statistically significant signals in the majority of the bands and bins, with the noisiest signals from bins of lowest masses and highest redshifts, and the most robust signals in those of the higher mass bins. Also, as expected from the beam size and noise properties of the maps, the MIPS $24 \mu \mathrm{m}$ and three SPIRE bands return stacked flux densities with the highest signal-tonoise, while the 70 and $160 \mu \mathrm{m}$ uncertainties are larger. The uncertainties at $1100 \mu \mathrm{m}$ are also higher, but that is largely a reflection of the area of the AzTEC field, which is half that of the other bands.

We note that the traditional stacking method, as anticipated from simulations (Section 3.3), returns systematically higher results, with the bias proportional to the strength of the clustering, which increases with increasing stellar mass. Considering this 


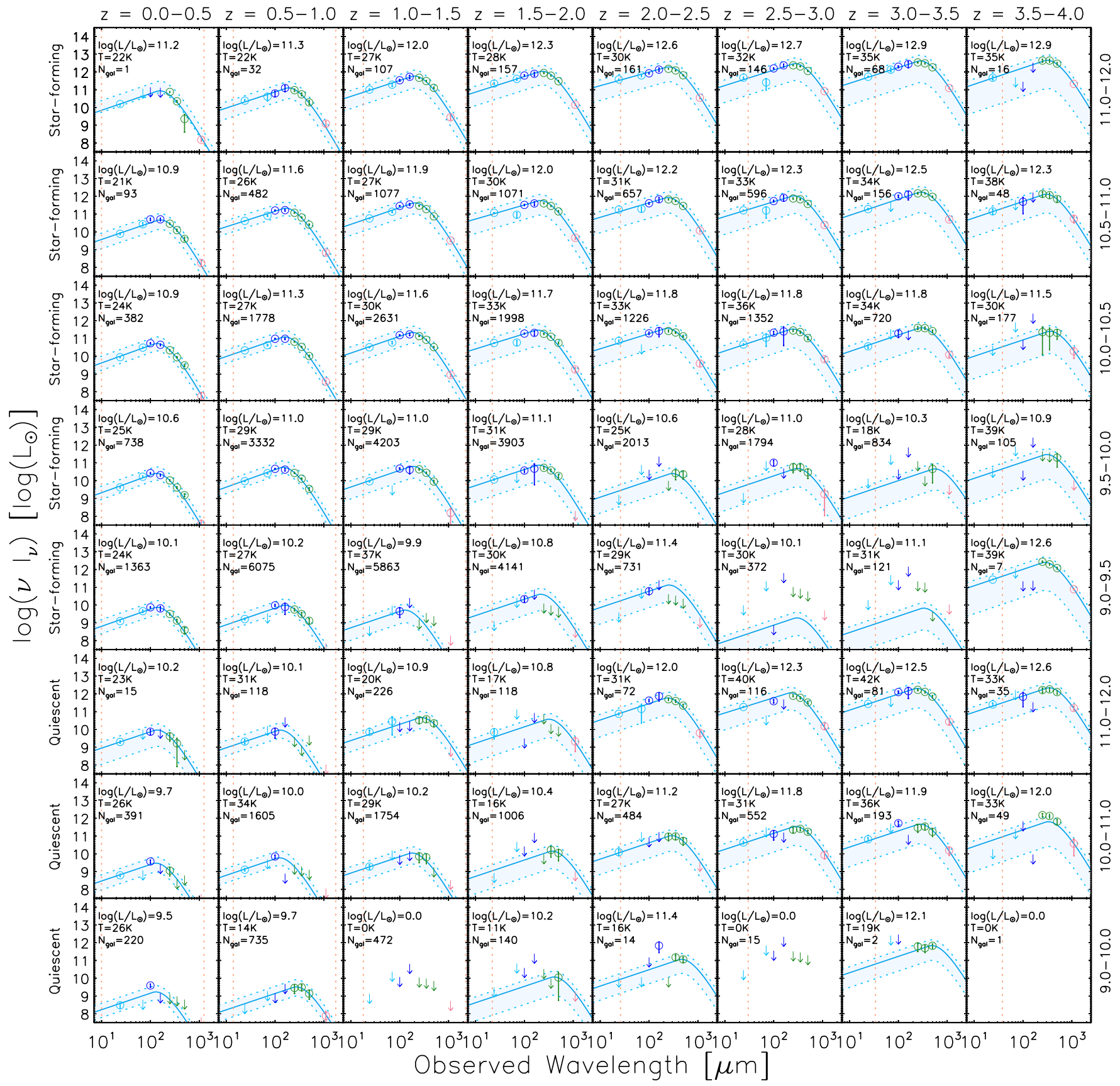

Figure 6. Stacked intensities $\left(\nu I_{\nu}\right)$ of mass-selected sources in the UDS field divided into bins of mass and redshift. Data at 24 and $70 \mu \mathrm{m}$ (light blue circles) are from Spitzer/MIPS; those at 100 and $160 \mu \mathrm{m}$ (dark blue circles) are from Herschel/PACS; those at 250, 350, and $500 \mu \mathrm{m}$ (green circles) are from Herschel/SPIRE; and those at $1100 \mu \mathrm{m}$ (red circles) are from AzTEC. The error bars represent the $1 \sigma$ Gaussian uncertainties estimated with the extended bootstrap method described in Section 3.4. Non-detections are shown as $2 \sigma$ upper limits plotted as downward pointing arrows. The median values of the redshift distributions are used to convert flux densities into rest-frame luminosities. The SED is modeled as a modified blackbody with a fixed emissivity index, $\beta=2.0$, and a power-law approximation on the Wien side with slope $\alpha=1.9$. The solid blue line in each panel is the best-fit SED, and the shaded region enclosed by dotted light-blue lines shows the systematic uncertainties due to the width of the redshift distribution (interquartile range), which dominates the error budget.

(A color version of this figure is available in the online journal.)

trend, any method that applies one correction for all stacked results should be viewed with suspicion.

Also notable is the significant contribution from galaxies identified as quiescent by their colors, a signal which is most prominent from the galaxies in the highest redshift bins. Their flux densities in all bands increase steadily with increasing redshift, to the point where at $z \gtrsim 3$, they are comparable to those of the most massive star-forming galaxies in the sample. It is likely that this is the result of misclassification of star-forming galaxies arising from low signal-to-noise photometry scattering their colors into the quiescent plane of the UJV diagram. We discuss this and other scenarios in Section 5.2.

\subsection{Best-fit SEDs}

Intensities, $\nu I_{\nu}$, are estimated from stacked flux densities and plotted in Figure 6 with detections shown as circles, while nondetections are shown as $2 \sigma$ upper limits. Stacked flux densities trace out the SED of thermally emitting warmed dust. While the shape of the SED is a superposition of many blackbody emitters of different temperatures (e.g., Wiebe et al. 2009), it has been 
shown to be well modeled as a modified blackbody of the form

$$
S_{v}=A v^{\beta} B(v, T),
$$

where $B(v, T)$ is the blackbody spectrum (or Planck function) with amplitude $A$, and $\beta$ is the emissivity index. The midinfrared exponential on the Wien side of the spectrum can be replaced with a power law of the form $f_{v} \propto v^{-\alpha}$, which is added by specifically requiring that the two functions and their first derivatives be equal at the transition frequency. Values for both $\beta$ and $\alpha$ in the literature range from 1.5 to 2 (e.g., Blain et al. 2003; Dunne et al. 2011; Viero et al. 2012), while we use $\beta=2$ and $\alpha=1.9$, which represent the mean values of the best fits of the individual SEDs. Note that for both $\beta$ and $\alpha$, we check that the exact values chosen does not significantly bias the result.

Our SED fitting procedure estimates the amplitude and temperature of the above template. For the SPIRE points, the SED fitting procedure (described in detail in Chapin et al. 2008) takes the width and shape of the photometric bands into account, as well as the absolute photometric calibration uncertainty in each band. Correlations due to instrumental noise are estimated and accounted for with a Monte Carlo procedure.

Correlated confusion noise must also be accounted for in the fit, as these correlations reduce the significance of a detection in single band. That is, not accounting for correlated noise in the measurements of, say, the three SPIRE bands, would lead to attributing additional weight in the overall fit to these data, potentially leading to a bias. This is discussed in more detail in Moncelsi et al. (2011) and Viero et al. (2012). We estimate the Pearson coefficients of the correlation matrix for all bands from the beam-convolved maps (Table 4).

Interquartile errors, which reflect the uncertainty in dimming due to the width of the redshift bins, are estimated from the distribution of redshifts over the full set of simulated catalogs generated as described in Section 3.4. Best-fit SEDs for each mass and redshift bin are shown in Figure 6 as solid blue lines; dotted blue lines and shaded regions represent the interquartile errors.

The best-fit SEDs serve several purposes. The first, and our primary purpose, is to infer the contribution from galaxies in each bin to the entire CIB (spanning the full range of wavelengths of our sample). Another purpose is to estimate infrared luminosities, as described in Kennicutt (1998), by integrating the SED between rest-frame 8 and $1000 \mu \mathrm{m}$ (shown as horizontal yellow dotted lines in panels of Figure 6). These are later used when quantifying the contribution to the CIB from galaxies classified as "normal," luminous, and ULIRGs (Section 4.6). Infrared luminosities can be used as an indicator of obscured star formation, a topic that will be explored in V. Arumugam et al. (in preparation). Finally, best-fit SEDs give a measure of the effective dust temperatures, which we discuss in Section 4.7. For reference, both temperatures and luminosities are listed in the panels of Figure 6.

\subsection{Total Resolved CIB}

We estimate the contribution to the CIB from our $K$-selected galaxy sample (first without correcting for incompleteness) by multiplying the emission from each bin $\left(v I_{v}\right)$ by the number of galaxies in that bin, and summing them together. Results are tabulated in the second column of Table 5, labeled "Total Stacking."

Next, corrections for incompleteness are made for samples that are more than $50 \%$ complete, by dividing each bin by its completeness estimate (drawn from Figure 2) before summing. Results are tabulated in the third column of Table 5, labeled "Completeness Corrected." The choice of 50\%, though somewhat arbitrary, is chosen because at that point the uncertainty in the incompleteness estimate is not yet greater than the correction. Note that the uncertainties associated with this correction are accounted for as part of the Monte Carlo simulation used to estimate the total uncertainties. We find that the completeness correction adds between $\sim 1 \%-3 \%$ to the total CIB. If we relax the completeness requirement and correct bins which are as little as $10 \%$ complete, the completeness correction rises to $\sim 8 \%-15 \%$ and the resolved CIB to $97 \%$ in the SPIRE bands. Although hardly robust, this at least suggests that some fraction of the remaining CIB originates from faint sources. This scenario is discussed in more detail in Section 5.4.1. In all subsequent CIB figures, plotted points are completeness-corrected unless otherwise noted, with the total contribution in each band plotted as pink squares.

Also plotted are estimates of the total CIB as measured by: Spitzer/MIPS at $24 \mu \mathrm{m}$ (diamond; Dole et al. 2006); IRAS at $60 \mu \mathrm{m}$ (boxes; Miville-Deschênes et al. 2002); Spitzer/MIPS at 24, 70, and $160 \mu \mathrm{m}$ (asterisks; Béthermin et al. 2010) as well as at 70 and $160 \mu \mathrm{m}$ (exes; Jauzac et al. 2011); WHAM at 100, 140, and $240 \mu \mathrm{m}$ (crosses; Lagache et al. 2000); and from COBE/ FIRAS spectra spanning 200-1200 $\mu \mathrm{m}$ (solid line; Lagache et al. 2000). Lower limits are shown as upward pointing arrows from Spitzer/MIPS at $24 \mu \mathrm{m}$ (Papovich et al. 2004) and at 70 and $160 \mu \mathrm{m}$ (Dole et al. 2006); SPIRE at 250, 350, and $500 \mu \mathrm{m}$ (Béthermin et al. 2012c); and SCUBA at $450 \mu \mathrm{m}$ (Serjeant et al. 2004) and $850 \mu \mathrm{m}$ (Smail et al. 2002). Last, plotted as lavender asterisks is the resolved CIB using $24 \mu \mathrm{m}$ priors from J. D. Vieira et al. (in preparation).

As previously described, the relationship between stacked fluxes from different bands is well approximated by a simple thermal dust SED. This can be used to roughly estimate the total resolved CIB between bands, as well as give us a better handle on the contribution from noisy bands. Thus, we estimate the total contribution to the CIB from summing the best-fit SEDs of Figure 6 (corrected for incompleteness), and report them in the fourth column of Table 5, labeled "Total Model SEDs." Comparing these measurements to the absolute CIB values listed in the last column of the same table, labeled "Reference," we find that our full sample resolves $(69 \% \pm 15 \%),(78 \% \pm 18 \%)$, $(58 \% \pm 13 \%),(78 \% \pm 18 \%),(80 \% \pm 17 \%),(69 \% \pm 14 \%)$, $(65 \% \pm 12 \%)$, and $(45 \% \pm 8 \%) \mathrm{nW} \mathrm{m}^{-2} \mathrm{sr}^{-1}$ at $24,70,100$, $160,250,350,500$, and $1100 \mu \mathrm{m}$, respectively.

\subsection{Contribution to the CIB in Broad Redshift Bins}

Plotted as open circles in the top panel of Figure 7 and tabulated in Table 6 are estimates of the contribution to the total resolved CIB in four redshift bins. The dot-dashed lines connecting the circles represent the equivalent summed SED fits. Notable is the striking dependence on the contribution to different bands from different redshifts - a result of negative $K$-correction-with the $z=0-1$ bin dominating the CIB at $\lambda \lesssim 160 \mu \mathrm{m}$, and the $z=1-2$ bin the chief contributor at $\lambda \gtrsim 250 \mu \mathrm{m}$.

Not surprisingly, as infrared luminosity is a tracer of obscured star formation (e.g., Kennicutt 1998), this behavior closely mimics the rapid rise in the star-formation history of the universe (e.g., Hopkins \& Beacom 2006; Behroozi et al. 2013b), peaking at $z \sim 1$, as well as the same general trends as the model predictions of Béthermin et al. (2011, top panel of Figure 11), 


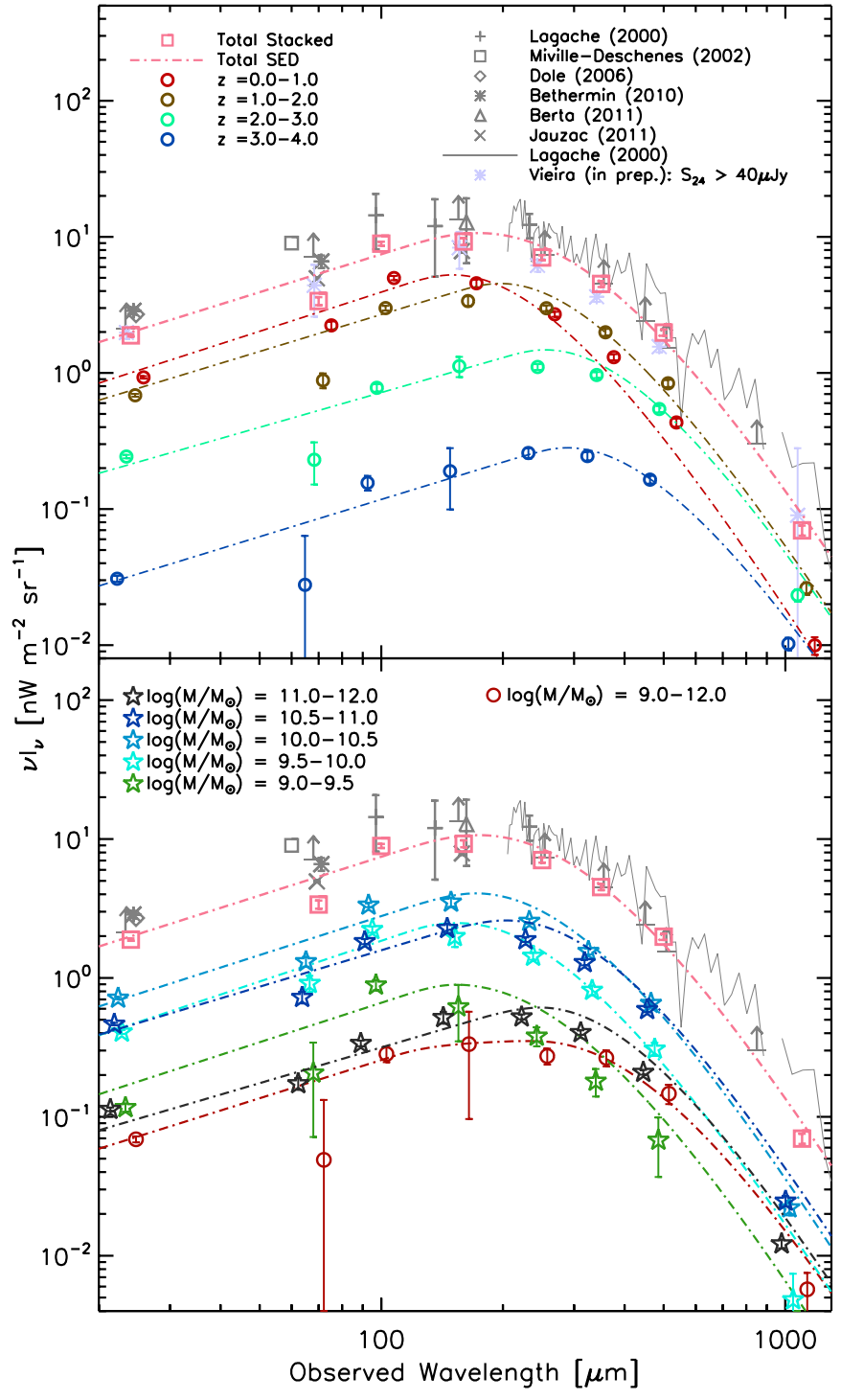

Figure 7. Top panel: contribution to the CIB from equally spaced redshift slices. Measured data are shown as circles with error bars, while best-fit SEDs are shown at dot-dashed lines. Also plotted as lavender asterisks is the resolved CIB using $24 \mu \mathrm{m}$ priors from J. D. Vieira et al. (in preparation). Bottom panel: contribution to the CIB in divisions of mass, with star-forming galaxies shown as stars and quiescent galaxies (with all mass bins combined) shown as circles. Both panels: also plotted is a selection of measurements of the total CIB in grey, from: Spitzer/MIPS at $24 \mu \mathrm{m}$ (diamond; Dole et al. 2006); IRAS at $60 \mu \mathrm{m}$ (boxes; Miville-Deschênes et al. 2002); Spitzer/MIPS at 24, 70 and $160 \mu \mathrm{m}$ (asterisks; Béthermin et al. 2010); and 70 and $160 \mu \mathrm{m}$ (exes; Jauzac et al. 2011); Herschel/PACS at $160 \mu \mathrm{m}$ (triangles; Berta et al. 2011); WHAM at 100, 140, and $240 \mu \mathrm{m}$ (crosses; Lagache et al. 2000); and COBE/FIRAS spectra from $\sim 200$ to $1200 \mu \mathrm{m}$ (solid line; Lagache et al. 2000). Lower limits are from Spitzer/MIPS at 24, 70, and $160 \mu \mathrm{m}$ (Papovich et al. 2004; Dole et al. 2006); SPIRE at 250, 350, and $500 \mu \mathrm{m}$ (Béthermin et al. 2012c); and SCUBA at 450 and $850 \mu \mathrm{m}$ (Smail et al. 2002; Serjeant et al. 2004). Our total CIB measurements and best-fit SEDs are shown in both panels as pink boxes and dot-dashed lines, respectively.

(A color version of this figure is available in the online journal.)

though it should be noted that the limits of the redshift bins are not identical. We explore similarities with models and agreement with measurements of the infrared luminosity density further in Section 4.6.

\subsection{Contribution to the CIB in Stellar-mass Bins}

We estimate the contribution to the CIB in divisions of stellar mass by summing completeness-corrected emission in rows of Figure 6, with star-forming and quiescent galaxies grouped separately, and tabulated in Tables 7 and 8, respectively. They are also shown in the bottom panel of Figure 7 as color-coded stars and circles (quiescent galaxies are all grouped together), with the dot-dashed lines representing the equivalent summed SED fits.

A significant part of the resolved CIB ( $\gtrsim 65 \%)$ at all wavelengths appears to originate from star-forming galaxies having stellar masses between $\log \left(M / M_{\odot}\right)=10.0-11.0$. The total contribution from more massive galaxies is $\sim 5 \%$, while that from less massive galaxies is $30 \%$. Galaxies from the $\log \left(M / M_{\odot}\right)=10.0-10.5$ bin provide the highest contribution everywhere; while those from the $\log \left(M / M_{\odot}\right)=10.5-11.0$ bin provide second highest contribution at $\lambda \gtrsim 160 \mu \mathrm{m}$, and those from the $\log \left(M / M_{\odot}\right)=9.5-10.0$ bin provide the second highest contribution at shorter wavelengths. Quiescent galaxies together, unsurprisingly, contribute very little to the total resolved background, of order $5 \%$.

Galaxies form in dark matter over-densities, or halos (e.g., Mo \& White 1996), with the peak efficiency for star formation in halos of $\log \left(M / M_{\odot}\right) \sim 12.0$ (e.g., Moster et al. 2010; Behroozi et al. 2010; Béthermin et al. 2012b; Viero et al. 2013b), which appears to be remarkably consistent throughout the age of the universe (Behroozi et al. 2013a). On the other hand, the stellar mass of the galaxies which formed in these halos, and the evolution of that relationship, is less certain. Wang et al. (2013) found stellar-to-halo mass values of $\sim 10^{-2}$ to $10^{-3}$ from to $z=0$ to 2 , which equates to $\log \left(M / M_{\odot}\right) \sim 10.4$ to 10.6 , largely consistent with our findings. We explore the luminosity density evolution of these same galaxies in the next section.

\subsection{Contribution to the CIB as a Function of Galaxy Luminosity}

Infrared luminosities, $L_{\mathrm{FIR}}$, are calculated by integrating the rest-frame SEDs between 8 and $1000 \mu \mathrm{m}$ (Kennicutt 1998). In Figure 8 we plot $L_{\mathrm{FIR}}$ as a function of redshift, in divisions of stellar mass, with star-forming and quiescent galaxies displayed as stars and circles, respectively, and open symbols represent bins whose completeness is greater than $50 \%$. Infrared galaxies have conventionally been classified by their luminosities into "normal" $\left(L<10^{11} L_{\odot}\right)$, luminous (luminous infrared galaxies, LIRG: $L=10^{11-12} L_{\odot}$ ) and ultra-luminous (ULIRG: $L=$ $\left.10^{12-13} L_{\odot}\right)$ infrared galaxy classes, illustrated in Figure 8 as horizontal dotted lines and right-handed labels.

We fit simple polynomials to $L\left(M_{i}, z\right)$ versus $z$ to each stellarmass bin, $i$, such that

$$
\log \left(L\left(M_{i}, z\right)\right)=\sum_{j=0}^{n} x_{i, j} z^{j}
$$

following the rule that the $i$ variables of each $x_{i, n}$ must additionally obey their own polynomial fit,

$$
x_{i, j}=\sum_{k=0}^{n} y_{j, k} \log \left(M_{i}^{k}\right)
$$

where the order of the polynomial fit, $n$, is 2 and 1 for star-forming and quiescent galaxies, respectively. This simple parameterization ties together the evolution of the luminosity with stellar mass and redshift, and allows us to fully explore the 


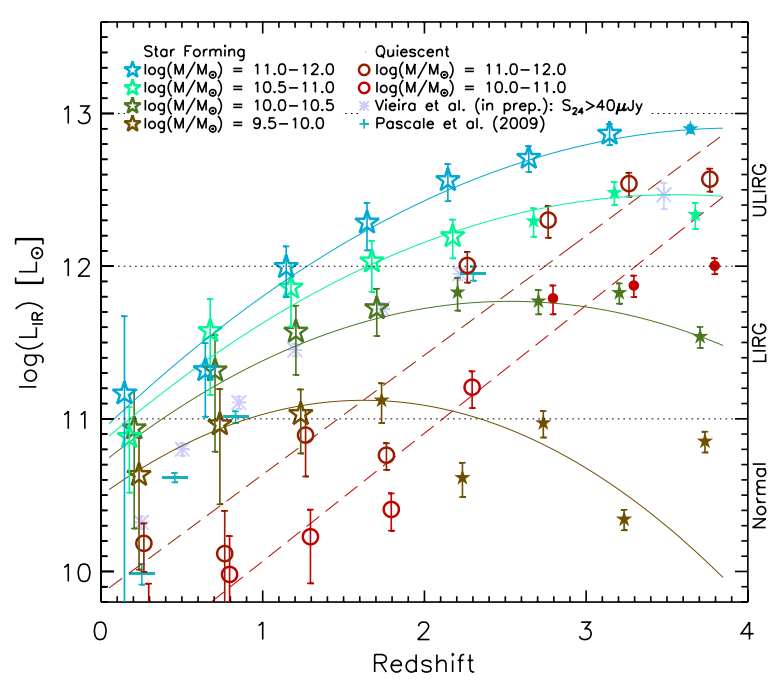

Figure 8. Luminosity vs. redshift in divisions of mass for star-forming (stars) and quiescent galaxies (circles). Open symbols represent bins with greater than $50 \%$ completeness. Polynomial fits to the data are plotted for star-forming (solid lines) and quiescent (dashed lines) galaxies. Notable is the rapid evolution in luminosity of the quiescent population, possibly due to noise in the optical photometry of higher redshift sources scattering star-forming galaxies into the quiescent section of the $U V J$ plane. Despite this enhanced luminosity, galaxies identified as quiescent provide only about $5 \%$ to the total CIB (Section 4.5). Results from stacking of $24 \mu \mathrm{m}$ selected sources from Pascale et al. (2009) and J. D. Vieira et al. (in preparation) are plotted as cyan crosses and lavender asterisks, respectively.

(A color version of this figure is available in the online journal.)

$L-M-z$ space. ${ }^{33}$ We find

$$
\begin{gathered}
\mathbf{y}_{\text {star forming }}=\left(\begin{array}{ccc}
-7.248 & 3.160 & -0.137 \\
-1.634 & 0.335 & -0.009 \\
-7.758 & 1.374 & -0.062
\end{array}\right), \\
\mathbf{y}_{\text {quiescent }}=\left(\begin{array}{cc}
2.672 & 0.624 \\
1.430 & -0.056
\end{array}\right),
\end{gathered}
$$

and plot them respectively as solid and dashed lines in Figure 8.

We find a rapid rise of luminosity with redshift for the most massive populations, and an apparent turnover at $z \gtrsim 2$ in the less massive ones; though we caution that incompleteness makes this turnover effect difficult to interpret. Particularly striking is the evolution of the luminosities of quiescent galaxies, since at lower than $z \sim 2$ they are barely detectable, while by $z \sim 3$ they are nearly as luminous as star-forming galaxies of similar mass. This is likely partially due to misclassification of starforming galaxies arising from low signal-to-noise photometry at high-redshift scattering galaxies into the quiescent part of the $U V J$ plane. Note that although the most massive galaxies at high redshift are very luminous, they make up a relatively small fraction of the full catalog and thus contribute only $\sim 4 \%$ of the total resolved CIB (see Section 4.5, and bottom panel of Figure 7). It is possible that some fraction of the dust heating is due to active galactic nuclei (AGN), as similar behavior has been seen in individually resolved objects at $24 \mu \mathrm{m}$ (e.g., Pérez-González et al. 2008b; Marchesini et al. 2010), though the optical SEDs of this population on average are not indicative of AGN. These scenarios are discussed in more detail in Sections 5.2 and 5.3.

\footnotetext{
33 The fitting functions to produce these curves are made available online.
}

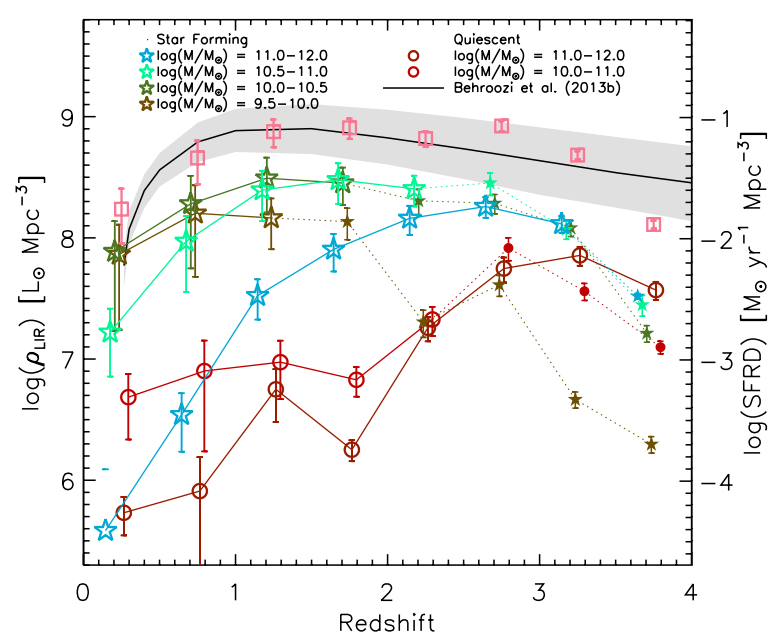

Figure 9. Luminosity density vs. redshift in divisions of mass for star-forming (stars) and quiescent galaxies (circles). Open symbols represent bins with greater than $50 \%$ completeness. We find a rapid rise in the contribution of the most massive galaxies to the luminosity density, as well as a steep decline in less massive galaxies, indicative of downsizing. Pink squares are totals for each redshift, which agree well with the model of Behroozi et al. (2013b).

(A color version of this figure is available in the online journal.)

Also shown are $24 \mu \mathrm{m}$ selected stacking results from (Pascale et al. 2009, cyan crosses) and J. D. Vieira et al. (in preparation, lavender asterisks). Their selection groups together galaxies of all masses and colors making a direct comparison difficult, yet the general trend of both stacks seem to agree reasonably well.

In Figure 9 we explore the contribution to the infrared luminosity density $\left(\rho_{\mathrm{L}}\right.$; e.g., Hopkins \& Beacom 2006; Le Floc'h et al. 2005; Rodighiero et al. 2010; Behroozi et al. 2013b) in divisions of stellar mass and color. Again, starforming and quiescent galaxies are displayed as stars and circles, respectively, and open symbols represent bins with greater than $50 \%$ completeness. We find that the contribution from the most massive galaxies (which we have already found account for less than $4 \%$ to the total CIB) evolves rapidly with redshift, such that by $z>2$ they are responsible for as much as their more abundant, less massive counterparts - a clear demonstration of downsizing (e.g., Cimatti et al. 2006; Fontanot et al. 2009).

Plotted as pink squares is the total $\rho_{\mathrm{L}_{\mathrm{IR}}}$, as well as a model prediction for the star-formation rate density (SFRD) from Behroozi et al. (2013b), converted to luminosity density using the Kennicutt (1998) relation

$$
\operatorname{SFR}\left[M_{\odot} \mathrm{yr}^{-1}\right]=1.728 \times 10^{-10} L_{\mathrm{IR}}\left[L_{\odot}\right],
$$

with an additional lowering by 0.23 dex to convert to a Chabrier (2003) IMF (e.g., Kriek et al. 2009). Equivalent values for the SFRD are shown for reference on the right-hand axis of Figure 9. We find relatively good agreement with the model, which is also in agreement with a host of different measurements (e.g., Rodighiero et al. 2010; Pérez-González et al. 2008a; Le Floc'h et al. 2005; Caputi et al. 2007; Casey et al. 2012; Burgarella et al. 2013; Magnelli et al. 2013) and models (e.g., Hopkins \& Beacom 2006).

In Figure 10 we explore the contribution to the CIB from "normal" galaxies, LIRGs, and ULIRGs. Data are constructed by summing intensities of bins corresponding to their luminosities as determined from the best-fit SEDs (Section 4.2). Short of $160 \mu \mathrm{m}$, the contribution from LIRGs and less-luminous galaxies is comparable, while at wavelengths longer than $160 \mu \mathrm{m}$, LIRGs clearly dominate the resolved CIB. The 


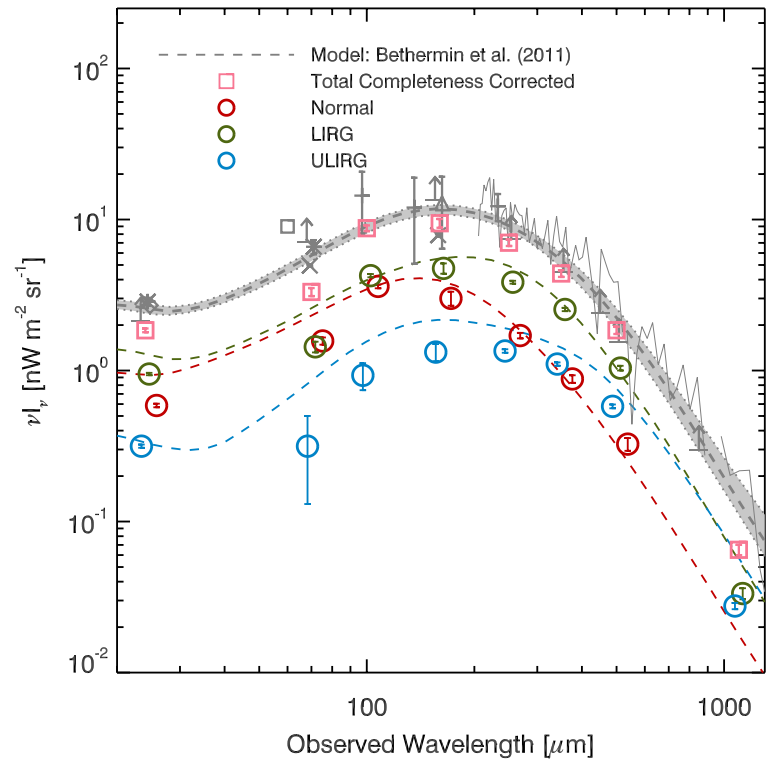

Figure 10. Contribution to CIB from "normal" galaxies ( $L<10^{11} L_{\odot}$ ), LIRGs $\left(L<10^{11-12} L_{\odot}\right)$, and ULIRGs $\left(L<10^{12-13} L_{\odot}\right)$. Normal galaxies and LIRGs contribute equally to make up most of the intensity at $\lambda \lesssim 70 \mu \mathrm{m}$, which is more sensitive to lower redshifts, while at longer wavelengths LIRGs and eventually ULIRGs contribute most to the signal. Also plotted are model predictions from Béthermin et al. (2010, Figure 13, bottom panel), with the LIRG and ULIRG predictions somewhat high. Although the model is a simple parametric fit to counts at multiple wavelengths, the high estimates for the LIRGs and ULIRGs lends weight to the suggestion that we are missing luminous, dust-obscured sources in our sample (Section 5.4.2).

(A color version of this figure is available in the online journal.)

contribution from $\log \left(L / L_{\odot}\right)<11$ galaxies falls rapidly at wavelengths greater than $160 \mu \mathrm{m}$, which may suggest a diminishing contribution from fainter populations at high redshift — which is again suggestive of downsizing — but it could also mean that fainter galaxies are simply being missed. The contribution from ULIRGs, which, as seen from Figure 8, are located at $z \gtrsim 1$, peaks at longer wavelengths, and is an order of magnitude lower than less-luminous galaxies at $\lambda \lesssim 160 \mu \mathrm{m}$. Note that if small numbers of exceptionally luminous sources, ultra-luminous or hyper-luminous infrared galaxies, have unusually high luminosities with respect to their stellar masses (i.e., high specific luminosities) this plot would fail to capture their distribution accurately.

Also overlaid in this figure are predictions from Béthermin et al. (2011, bottom panel of Figure 11), a parametric backwardevolution model fit to counts at multiple wavelengths. The general trends are well reproduced, while in detail, ULIRGs fall short of model predictions. As we discuss Section 5.4.2, this may be an indication that highly dust-obscured galaxies are missing from our optical/NIR-based, mass-selected catalog (e.g., Dey et al. 1999).

\subsection{Average Temperature Evolution for Star-forming Galaxies}

In Figure 11 we plot temperatures derived from our best-fit SEDS as a function of redshift (left panel), infrared luminosity (center panel), and stellar mass (right panel) for star-forming galaxies divided into stellar-mass (left panel) or redshift bins (center and right panels). We emphasize that the reported temperatures are tied to the simple modified blackbody used to derive them (Section 4.2), and that if another model had been used (e.g., a $\beta$ value of 1.5 instead of 2, a different opacity model, or a two component SED similar to that used by Dunne $\&$ Eales 2001), slightly different temperature values would have been derived (also see Casey 2012). However, the trends in the temperatures-either with redshift or with mass-should be relatively free of bias due to the model adopted. Moreover, since our relatively high signal-to-noise measurements bracket the peak of the thermal SED, our ability to identify these trends is robust.

We compare with temperature measurements of other galaxies—some FIR-selected, others NIR-selected—noting that

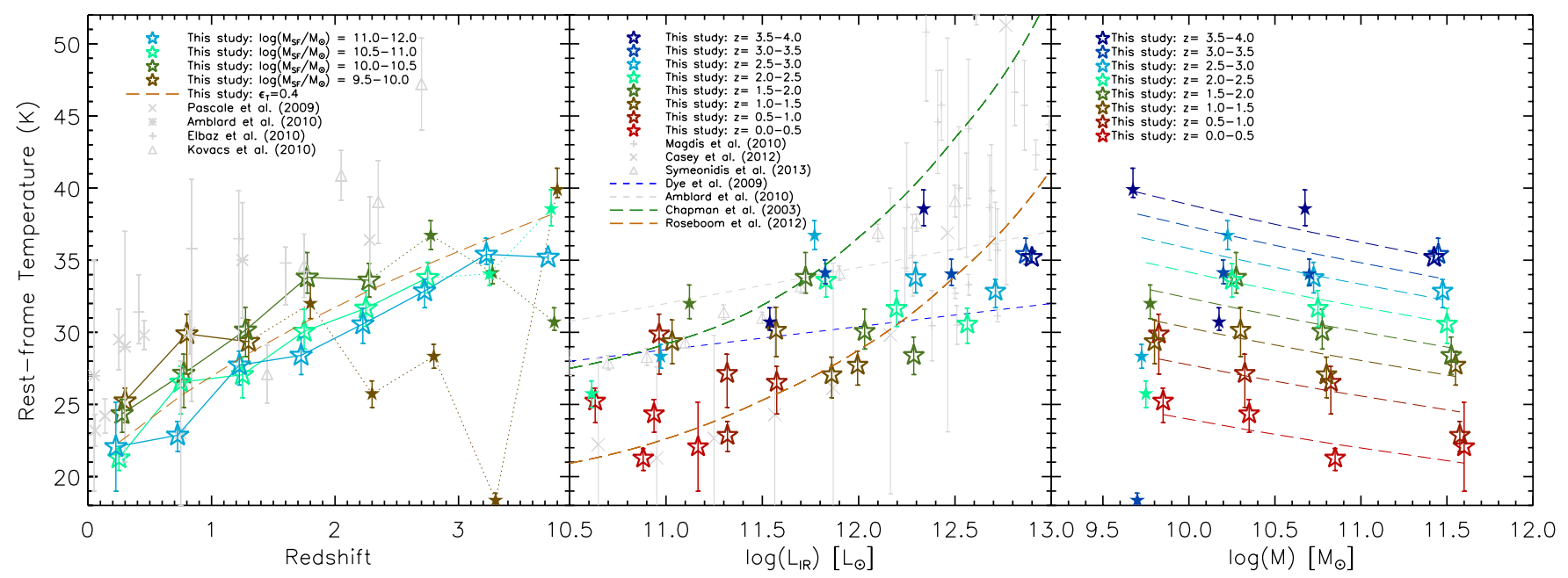

Figure 11. Average temperatures derived from the best-fit, modified blackbody SEDs vs. redshift in the left panel; infrared luminosity in the center panel; and stellar mass in the right panel. Open symbols represent bins with higher than 50\% completeness. Left panel: temperatures of galaxies of all stellar masses are found to evolve strongly with redshift. This evolution can be described as a power law with slope $\epsilon=0.4 \pm 0.1$ (orange dashed line). Also plotted are measurements from Pascale et al. (2009, pink exes); Amblard et al. (2010, asterisks); Elbaz et al. (2010, crosses), and Kovács et al. (2010, triangles). Center panel: the full ensemble of galaxy temperatures is shown to obey the canonical $L-T$ relation, described for local infrared galaxies by Chapman et al. (2003b, green dashed line) and at higher redshift by Roseboom et al. (2012, red dashed line). Also plotted are best-fits to BLAST and SPIRE sources from Amblard et al. (2010, gray dashed line) and Dye et al. (2009, blue dashed line), respectively, and measurements from Magdis et al. (2010, crosses); Casey et al. (2012, exes); and Symeonidis et al. (2013, triangles). Right panel: conversely, the temperatures of galaxies appears to decrease with increasing stellar mass (and thus increasing $L_{\mathrm{IR}}$ ). Shown as dashed lines are tied power-law fits to the data at each redshift.

(A color version of this figure is available in the online journal.) 
because our sample is mass-selected, we anticipate there to be discrepancies due to the selection functions of the different sets of galaxies. In the leftmost panel we see a clear trend of an increase of temperature with redshift for galaxies of all stellar masses. This trend is also seen in stacked measurements from Pascale et al. (2009, pink exes), from submillimeter-selected galaxies from Amblard et al. (2010, asterisks) and Elbaz et al. (2010, crosses), and Spitzer-selected galaxies from Kovács et al. (2010, triangles). The systematic offset of our mass-selected sample from these may partly be a result of the model fitted, and partly due to the fact that IR-selected sources will consequently be more luminous.

Following Addison et al. (2013), we fit the function

$$
T=T_{0}\left(\frac{1+z}{1+z_{\mathrm{T}}}\right)^{\epsilon_{\mathrm{T}}},
$$

where $T_{0}=27 \mathrm{~K}$ and $z_{\mathrm{T}}=1$ are the pivot temperature and redshift, respectively. This simple relationship is central to current halo models of the CIB (e.g., Addison et al. 2013; De Bernardis \& Cooray 2012; Shang et al. 2012; Viero et al. 2013 b), yet remains poorly constrained, with values ranging from $\epsilon_{\mathrm{T}}=0.16 \pm 0.02$ (Viero et al. 2013b, Model 2) to $\epsilon_{\mathrm{T}}=0.75 \pm 0.10$ (Addison et al. 2013). We find $\epsilon_{\mathrm{T}}=0.4 \pm 0.1$, in good agreement with the CIB models presented in Viero et al. (2013b, Model 3) and the Planck Collaboration et al. (2013).

In the central panel we explore the well established Luminosity-Temperature ( $L-T)$ relation (e.g., Dunne et al. 2000, 2011; Dunne \& Eales 2001; Dale et al. 2001; Dale \& Helou 2002; Chapman et al. 2003a; Chapin et al. 2009; Hwang et al. 2010; Magdis et al. 2010; Roseboom et al. 2012; Magnelli et al. 2013), comparing to relations measured by Chapman et al. (2003b, green dashed line), Roseboom et al. (2012, orange dashed line), Dye et al. (2009, blue dashed line), and Amblard et al. (2010, grey dashed line), along with NIR-selected galaxies from Magdis et al. (2010, crosses), and FIR-selected galaxies from Casey et al. (2012, exes) and Symeonidis et al. (2013, triangles).

We find poor agreement with the shallow slopes of Dye et al. (2009) and Amblard et al. (2010), which may be a reflection of the shallow nature of the submillimeter data used in those studies (i.e., BLAST and H-ATLAS, respectively), as well as the selection criteria (e.g., $3 \sigma$ detections in three SPIRE or PACS bands plus a $5 \sigma$ detection in SDSS or GAMA). On the other hand, we find generally good agreement with the trends reported locally in Chapman et al. (2003b) and at higher redshift by Roseboom et al. (2012); with overall values better described by the latter. We also find that our measurements are consistent with the mean values of resolved, SPIRE-selected sources from Casey et al. (2012), but that they are offset from those of Symeonidis et al. (2013).

However, we notice that our incomplete bins (filled stars) have systematically higher temperatures, suggesting that incomplete samples in the optical/NIR select hotter sources. This appears to be the case for the sources selected at NIR wavelengths by Magdis et al. (2010). This could also then mean that the selection criterion of Symeonidis et al. (2013, $24 \mu \mathrm{m}$ sources with $3 \sigma$ detections at $160 \mu \mathrm{m}$ and either 100 or $250 \mu \mathrm{m}$ ) favors sources with higher temperatures - in contrast to the missing "hot dust" ULIRG bias typically associated with submillimeter to millimeter sources (e.g., Eales et al. 2000; Blain et al. 2004; Chapman et al. 2004, 2008; Casey et al. 2009; Magdis et al. 2010; Magnelli et al. 2010; Hayward et al. 2012). Again, the small discrepancies between these different measurements is likely attributable to a combination of selection effects and model-based systematic bias (see also Magnelli et al. 2012).

Also of note in the central panel of Figure 11 is that in any one redshift bin the temperature appears to decrease somewhat with increasing luminosity. Similarly, in the rightmost panel we see that the temperature decreases for increasing stellar mass - particularly at lower redshifts-which follows since stellar mass and luminosity are strongly correlated (Figure 8). We explore the evolution of temperature with stellar mass and redshift by fitting the relations with tied power-law fits, i.e.,

$$
T(M, z)=A_{z} M_{z}^{\alpha_{\mathrm{T}, z}}
$$

where power laws must obey the rule that the amplitudes and slopes of the fits are also fit by power laws

$$
\begin{aligned}
& A_{z}=A_{z, 0}+A_{z, 1}(1+z)^{A_{z, 2},}, \\
& \alpha_{T}=\alpha_{T, 0}+\alpha_{T, 1}(1+z)^{\alpha_{T, 2},}
\end{aligned}
$$

where $\mathbf{A}=[-439.83,578.93,0.11]$ and $\alpha=[-0.81,2.84 \times$ $\left.10^{-5}, 3.55\right]$, which amounts to steady increase of temperature with redshift, a mild anti-correlation of temperature with stellar mass, but a negligible change of the slope of the anti-correlation over time.

There are several possible explanations for this apparent anticorrelation between stellar mass and temperature. Dust creation is thought to be dominated by supernovae ( $\mathrm{SNe}$ ), and to a lesser extent, evolving main sequence stars on the asymptotic giant branch (AGB; e.g., Michałowski et al. 2010a, 2010b). Since infrared luminosity (an established proxy for SFR; e.g., Kennicutt 1998) and dust mass (e.g., Santini et al. 2010; Skibba et al. 2011) are strongly correlated with stellar mass, it follows that there would be more dust extended over a larger volume in more massive galaxies (e.g., Menéndez-Delmestre et al. 2009). Also plausible is the possibility that less massive galaxies are more susceptible to stripping of their gas and dust (e.g., Abadi et al. 1999; McCarthy et al. 2008), which Rawle et al. (2012) show, can lead to higher temperatures.

Thus, our findings appear to be in tension with the local $(L-T)$ relation for SMGs, which may again be a function of the stellarmass selection versus far-infrared selection. Case in point: Hayward et al. (2013) show that galaxies with temperatures above roughly $40 \mathrm{~K}$ are universally starbursts (e.g., Hernquist 1989) as opposed to quiescently star forming (e.g., Davé et al. 2010). As we already showed in Section 4.6 and Figure 10, there are indications that this is exactly the population that is being missed.

\subsection{Redshift Distribution of the Resolved CIB}

The redshift distribution of the resolved CIB emission, $d\left(v I_{v}\right) / d z$, is measured by summing the completeness-corrected intensities of all stellar-mass bins separately in each band, and is plotted Figure 12 as upward pointing triangles, signifying that they are lower limits. The peak intensities shift from $z \sim 0.5-2$ with increasing wavelength, indicative of the peak of star formation occurring at $z \sim 1-2$ (e.g., Hopkins \& Beacom 2006; Behroozi et al. 2013b; Wang et al. 2013), and the redshift sensitivity of the different bands.

Also plotted are predictions for the entire CIB from a selection of recently published models. The Béthermin et al. (2012a) model in particular, which is based in part on stacking measurements with $24 \mu \mathrm{m}$ priors (Béthermin et al. 2012c), 


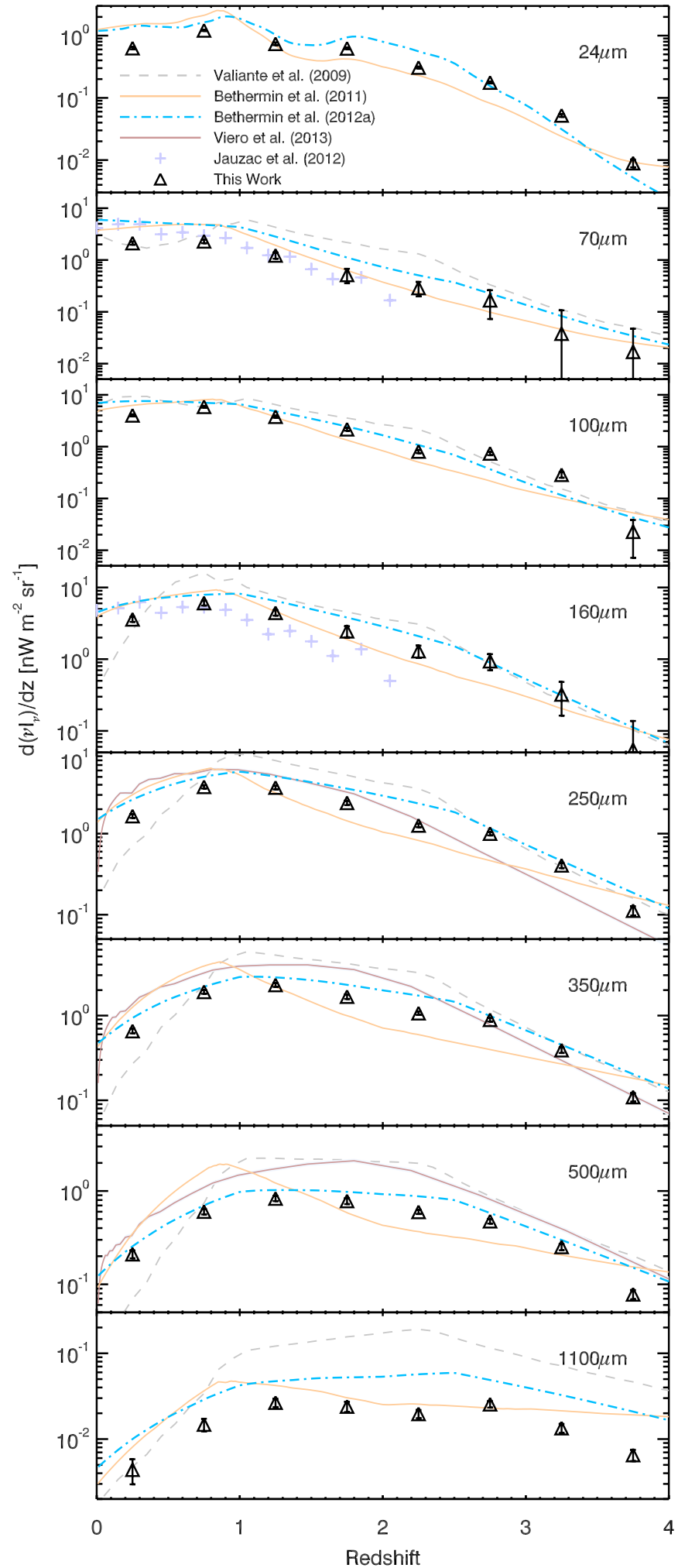

Figure 12. Redshift distribution in different bands of the resolved CIB emission, $d\left(v I_{v}\right) / d z$, in roughly spaced redshift slices. Over plotted are models from Valiante et al. (2009, dashed gray); Béthermin et al. (2011, solid yellow); Béthermin et al. (2012a, dot-dashed blue); and Viero et al. (2013b, solid brown at 250, 350, and $500 \mu \mathrm{m}$ ); as well as published data from Jauzac et al. (2011, lavender crosses at 70 and $160 \mu \mathrm{m}$ ). The Béthermin et al. (2012a) model, which is determined from stacking $24 \mu \mathrm{m}$ priors, fits remarkably well, suggesting a strong correlation between our different sets of catalogs.

(A color version of this figure is available in the online journal.) describes the measured distribution extremely well. However, we caution that since the completeness is a strong function of redshift (see Figure 2), it is not expected that the remaining CIB be distributed evenly in redshift.

This measurement has multiple applications. For example, CIB anisotropy measurements (e.g., Lagache et al. 2007; Viero et al. 2009, 2013b; Hall et al. 2010; Amblard et al. 2011; Planck Collaboration et al. 2011, 2013) are typically interpreted with halo models (e.g., Peacock \& Smith 2000; Cooray \& Sheth 2002), which assign intensities to dark matter halos a function of halo mass, redshift, and SED of thermal dust emission. These models find significant degeneracies between SEDs and the redshift distribution of the CIB (e.g., Addison et al. 2012; Shang et al. 2012), or halo bias and redshift distribution of the CIB (e.g., Holder et al. 2013), limiting their interpretive power. The measurements of $d\left(v I_{v}\right) / d z$ reported here, as well as the $T-M-z$ dependence of the thermal SED from Section 4.7, provide powerful constraints for future models.

\section{DISCUSSION}

\subsection{Incompleteness Bias}

The SIMSTACK technique returns an unbiased estimate of the flux density when all potentially correlated sources are accounted for. When sources are missing, either because they are too dust-obscured or intrinsically faint to detect, their unaccounted-for flux may potentially bias the result. While the UDS is an exceptionally deep survey, with greater than $90 \%$ completeness at $z \sim 3.5$ and $\log \left(M / M_{\odot}\right)=10.5$, it is possible that the absence of lower-mass galaxies, particularly at higher redshifts, could affect the results. We explore the severity of a potential bias with the following simulation.

Restricting ourselves to star-forming galaxies, we first simultaneously stack all mass bins at a single redshift and record their fluxes. Next, we remove the lowest mass bin and stack again, comparing the fluxes in the remaining bins to those of the initial stacked fluxes. We repeat this until only the highest mass bin remains. Results are as we might expect: the removal of bins increases the stacked fluxes of the remaining bins in a systematic way, from negligibly little for one missing bin, to as much as 3\%-22\% for the highest-mass bin alone; and that the level of the bias increases with beam size. For the case of two mass bins removed - roughly equivalent to the scenario of our highest redshift bin-the bias ranges over $0.2 \%-4.0 \%$, from smallest to largest beam size.

What does this mean for resolving the background? Although missing sources boost the fluxes of those remaining, the sum of their missing flux counteracts the boosting, resulting in a marginal bias. Our simulations confirm this, with the removal of up to three bins having negligible impact.

\subsection{Contribution from Quiescent Galaxies}

Here we briefly discuss the small contribution $(\sim 5 \%)$ to the total resolved CIB from galaxies classified as quiescent. The majority of this contribution originates at $z \gtrsim 2$, in contrast with the rest of the CIB, which comes mostly from star-forming galaxies at $z=0-2$. Indeed, at $z \gtrsim 2$ the most massive quiescent galaxies $\left(M=10^{11-12} M_{\odot}\right)$ are ULIRGs, and the next most massive $\left(M=10^{10-11} M_{\odot}\right)$ are LIRGs.

The simplest explanation is that star-forming galaxies are being misclassified as quiescent galaxies, particularly at $z \gtrsim 2.5$, 
where their signal-to-noise in the optical is lower, and their colors thus more likely to be scattered into the quiescent part of the UVJ plane. But dig a little deeper and that scenario alone seems unlikely, as to achieve ULIRG-like luminosities on average, either all quiescent galaxies at the highest redshifts must be misclassified ULIRGs-i.e., no passive quiescent galaxies past redshift of $\sim 2.5$ - or that the fraction that is misclassified would need to be incredibly luminous and abundant (e.g., $L \sim 2-4 \times 10^{12} L_{\odot}$ for $50 \%$ contamination, $L \sim$ $4-8 \times 10^{12} L_{\odot}$ for $25 \%$ contamination, etc.); both of which would be wildly inconsistent with the latest measurements of the stellar-mass function (Muzzin et al. 2013a; Ilbert et al. 2013).

Alternatively, it might be that interlopers misclassified in redshift as well as color are boosting the flux. This would result in a slightly hotter SED in the rest frame-which we do see-and significant boosting of $v I_{v}$ without the need for excessively luminous sources. However, we have already seen that as a result of negative $K$-correction, flux densities are roughly flat with redshift in the submillimeter bands (e.g., Figure 5 ), so that only relatively local (i.e., $z \lesssim 0.5$ ) sources would be capable of boosting the flux densities enough to account for the increased luminosities. But as that is also where photo- $z$ are most reliable (e.g., Brammer et al. 2008; Quadri \& Williams 2010), it unlikely that this is taking place.

Aside from measurement errors, it is worth noting that there are possible physical explanations for the FIR emission. Because the star formation in quiescent galaxies can only have shut off very recently at these redshifts, there may still be a strong interstellar radiation field heating the dust. For example, Fumagalli et al. (2013) explore whether not supposedly quiescent galaxies (also selected with the $U V J$ technique) are indeed dead. They split up their sample into five redshift bins and select only quiescent galaxies with stellar masses greater than $\log \left(M / M_{\odot}\right)>10.3$, finding $\log \left(L / L_{\odot}\right) \sim 9.5-10.5$ over the redshift range $0.3<z<2.5$. These luminosities are largely consistent with our findings - both in amplitude and in the evolution of that amplitude-but fall short of the redshift range where we find passive galaxies with ULIRG-like luminosities. They conclude that circumstellar dust from AGB stars (e.g., Knapp et al. 1992; Lançon \& Mouhcine 2002; Piovan et al. 2003), and cirrus heated by old stellar populations (e.g., the so-called cirrus heating Kennicutt 1998; Salim et al. 2009), are probably responsible for much of the IR emission in their sample, and that the SFRs in these galaxies is some 20-40 times lower than in their star-forming counterparts.

Another possibility is that there is FIR emission associated with embedded AGNs in highly obscured red galaxies at high redshift (e.g., Polletta et al. 2006, 2008; Daddi et al. 2007; Alexander et al. 2008; Kirkpatrick et al. 2012). AGNs are found to have $\operatorname{luminosities} \log \left(L / L_{\odot}\right)>12-13$ at $z>1$ (e.g., Pope et al. 2008; Dai et al. 2012), which would be enough to explain the rising luminosity with redshift, particularly since the evolution of the AGN fraction follows a similar trend (e.g., Alexander et al. 2005). We explore this scenario in more detail in the next section.

Finally, it might just be possible that increasing levels of star formation is present but so heavily obscured that the contribution from young stars to the rest-frame $J$-band luminosity is small. There are examples of high-redshift passive galaxies with $24 \mu \mathrm{m}$ flux suggesting excess FIR emission, (e.g., Marchesini et al. 2010; Pérez-González et al. 2008b); as well as optically selected red-and-dead galaxies stacked in the submillimeter showing excess flux, possibly indicating star formation (Viero et al. 2012).

\subsection{Active Galactic Nuclei and the High $\chi^{2}$ Sample}

AGNs have been found to contribute to the infrared luminosities of both passive and star-forming galaxies at levels between 3 and 37\% (e.g., Pope et al. 2008; Mullaney et al. 2011; Juneau et al. 2013). While submillimeter colors alone are inadequate for identifying AGNs (e.g., Hatziminaoglou et al. 2010; Kirkpatrick et al. 2013), the mid-infrared has been established as a sensitive probe of galaxy type (e.g., Brandl et al. 2006; Farrah et al. 2007), with the presence of an exponentially rising SED a robust signature of AGNs (Lacy et al. 2004; Richards et al. 2006; Hickox et al. 2007; Assef et al. 2010; Mullaney et al. 2011; Stern et al. 2012; Feltre et al. 2013). When spectra are not available, it has been shown that higher 22-24 $\mu$ m flux densities correlates with higher AGN fractions (Daddi et al. 2007; Lee et al. 2010; Murphy et al. 2011; Bridge et al. 2013).

We look for evidence of AGNs in the roughly 2700 sources that were otherwise poorly fit with standard SEDs by adding an AGN template (e.g., Assef et al. 2010) to the fit, and find that approximately half of the fits are significantly improved. Next, we stack them separately from-but simultaneously with - the star-forming and quiescent galaxies to look for further indications of AGNs. We find that the contribution to the CIB from these sources is $\lesssim 4 \%$, and is distributed over the same redshift range as the star-forming galaxies. Additionally, we find that the SEDs are on average $\sim 10 \mathrm{~K}$ hotter, an indication that emission from hot dust around the AGN (e.g., RowanRobinson \& Crawford 1989; Lacy et al. 2004) is contributing to the thermal SED at shorter wavelengths (e.g., Kirkpatrick et al. 2012; although also see Feltre et al. 2013). We also find that the $24 \mu \mathrm{m}$ flux density exceeds that of the other galaxies of similar mass, again, a strong signature of AGNs (e.g., Daddi et al. 2007; Lee et al. 2010).

While intriguing, fully understanding the contribution from AGNs is complicated and subtle, and lies beyond the scope of this paper. It is worth noting that because AGN emission should exist at varying levels in all galaxies (e.g., Juneau et al. 2013), the total contribution to the CIB attributable to AGNs may in fact be significant. A full treatment of this class of sources requires exploring this threshold with better mid-infrared diagnostics and fits using a full set of AGN templates will be explored in detail in L. Moncelsi et al. (in preparation).

\subsection{Where is the Missing CIB?}

Based on our best-fit SEDs, we resolve $2.0 \pm 0.5,5.2 \pm 1.2$, $7.4 \pm 1.7,10.7 \pm 2.5,8.4 \pm 1.8,4.54 \pm 1.0,1.7 \pm 0.3$, and $0.1, \pm 0.02 \mathrm{nW} \mathrm{m}^{-2} \mathrm{sr}^{-1}$ at $24,70,100,160,250,350$, 500 , and $1100 \mu \mathrm{m}$, respectively. This accounts for between $(45 \% \pm 8 \%)$ and $(80 \% \pm 17 \%)$ of the CIB, with the lowest fractions resolved at the longest (AzTEC) wavelengths, and the highest fractions in the three SPIRE bands. This is similar to the resolved fraction found by Marsden et al. (2009)_although they likely suffered from a bias due to neglecting clustering within the large BLAST beams (Pascale et al. 2008) —and by J. D. Vieira et al. (in preparation) and Béthermin et al. (2012c) at far-infrared/submillimeter wavelengths.

Between $(55 \% \pm 8 \%)$ and $(20 \% \pm 17 \%)$ of the CIB thus appears to be unaccounted for by our $K$-selected sample. It is notable that the measurements for the absolute CIB are themselves uncertain at the $\sim 25 \%$ level, so that formally our measurements could be said to be consistent with the CIB in 
any one band. However, considering that they fall below the nominal CIB in every band, the quoted percentage should be a fair estimate.

Besides the possibility that the absolute CIB is not entirely of extragalactic origin, the two most obvious candidates for the missing fraction are: (1) a large number of low-mass, intrinsically faint sources undetected by the UDS survey; and (2) IR luminous, potentially massive but unusually dust-obscured sources also missed by the UDS selection. We will now discuss these possibilities.

\subsubsection{Low-mass Faint Sources}

Our sample is selected at $K_{\mathrm{AB}} \leqslant 24$, reaching a source density on the sky of $\sim 36 \mathrm{arcmin}^{-2}$. Fainter, lower-mass sources missed by the catalog certainly exist (i.e., the faint end of the luminosity function, e.g., Muzzin et al. 2013a; Ilbert et al. 2013), but could they contribute enough intensity to make up the remaining CIB?

If we assume that there are as many undetected sources as there are detected, and that at, say, $500 \mu \mathrm{m}$, their flux densities are $0.1 \mathrm{mJy}$ (i.e., just below that of the current lowest mass bin) at all redshifts (not unreasonable given negative $K$-correction, e.g., see Figure 5), that would add $\sim 0.25 \pm 0.05 \mathrm{nW} \mathrm{m}^{-2} \mathrm{sr}^{-1}$, i.e., another $\sim(10 \% \pm 2 \%)$, to the CIB. This behavior mirrors the behavior demonstrated in Section 4.3, where aggressively correcting for completeness brought the CIB to within $5 \%$ of the nominal total. We thus conclude that some fraction is probably due to the faint population, though without a full simulation, or deeper data, it is difficult to say how much.

\subsubsection{Dust-obscured Sources}

There exists sources-for example submillimeter galaxies - that are incredibly luminous in the infrared (e.g., $L_{\mathrm{IR}}>10^{12} L_{\odot}$ ) but so dust-obscured that they are often very faint at UV/optical wavelengths (Smail et al. 1997; Barger et al. 1998; Hughes et al. 1998; Blain et al. 2002). For example, Dey et al. (1999), observing a $\log \left(L / L_{\odot}\right)=12.8$ ULIRG at $z=1.44$ with the Wide Field Planetary Camera 2 (Holtzman et al. 1995) on the Hubble Space Telescope with the F814W filter $(\lambda=7930 \AA)$ measured a magnitude of $24.6 \pm 0.1$ [AB]; i.e., this source would probably not have made our cut. These sources tend be irregular galaxies-likely due to a recent or ongoing merger (Hernquist 1989; Hayward et al. 2013)_forming stars in bursts rather than at a steady rate governed by the infrared "main sequence" for star-forming galaxies (e.g., Noeske et al. 2007; Elbaz et al. 2011).

A hint that luminous but obscured sources might be going undetected comes from Figure 10, where the resolved fraction of ULIRGs is in disagreement with the Béthermin et al. (2011) model, while less-luminous sources appear to be in quite good agreement. Since the sum of the three colored curves resolves the full CIB, it may well be that this missing component is rather significant.

Likely better tracers of highly dust-obscured star-forming galaxies are $24 \mu \mathrm{m}$ sources, which, as already mentioned, have been used by several groups as positional priors. For example, Dole et al. (2006) found that $24 \mu \mathrm{m}$ selected sources make up $70 \%$ of the CIB at 70 and $100 \mu \mathrm{m}$; while Berta et al. (2011) found they could explain between $58 \%$ and $64 \%$ of the CIB at 100 and $160 \mu \mathrm{m}$; and Béthermin et al. (2012c) resolve $70 \%$ of the background at 250,350, and $500 \mu \mathrm{m}$. However, a considerable fraction of the emission at $24 \mu \mathrm{m}$ is shown to originate from galaxies hosting AGNs (e.g., J. D. Vieira et al. in preparation).
As we discussed in Section 5.3, the inclusion of high $\chi^{2}$ sources increased the stacked $24 \mu \mathrm{m}$ flux density more than at other bands. And although we resolve about the same amount of the CIB, it does not mean we are resolving the same sources. It is possible that other faint AGNs are being missed, particularly at high redshift, where the AGN contribution dominates. The potential thus exists that we are missing a significant contribution from dust-obscured galaxies. In the future, identifying $24 \mu \mathrm{m}$ and other sources missed by our $K$-selected catalog — and stacking them simultaneously with our optically selected sources — would be a natural extension of this work.

\section{SUMMARY}

A complete understanding of galaxies and galaxy evolution requires characterizing their full SEDs, a goal which has to-date been technically challenging, as galaxies in the submillimeter are mostly unresolved. And for those that are resolved, large instrumental beams makes counterpart identification laborious. Here we attempted to statistically connect galaxies selected in the $K$-band to those that make up the CIB by stacking sources grouped into bins of mass, redshift, and color-presenting and making public our own SIMSTACK algorithm.

We found that between $(45 \% \pm 8 \%)$ and $(80 \% \pm 17 \%)$ of the total CIB is resolved by our catalog, and that the bulk originates from $z=0-2$ and $\log \left(M / M_{\odot}\right)=10.0-11.0$. Sources at higher redshifts contribute more at longer wavelengths, a consequence of the peak of the SED redshifting into the submillimeter/ millimeter bands (i.e., negative $K$-correction). Higher-mass LIRGs and ULIRGs are seen to contribute at longer wavelengths (i.e., higher redshift), which shifts to lower mass LIRGs and "normal" star-forming galaxies at shorter wavelengths.

We find that the luminosities of all galaxies rises rapidly with increasing redshift, but whereas they continue to rise until $z=4$ for the most massive galaxies, there is an apparent turnover at $z \gtrsim 2$ in galaxies of $\log \left(M / M_{\odot}\right) \lesssim 10.5$. We further find that while galaxies identified as quiescent by their colors have very little emission at low redshift, beyond $z \geqslant 2$ they evolve even more rapidly than their star-forming counterparts. This is likely due, to some extent, to limitations in the $U V J$ color selection at higher redshifts resulting from noisy photometry, though the origin of the entirety of this flux is still not certain, and will be the subject of future work. Last, galaxies whose photometry is poorly fit by standard SEDs appear to have a clear association with AGNs; having comparable luminosities but higher temperatures as star-forming galaxies of the same stellar mass.

Our work pushes the boundaries of characterizing low-mass and high-redshift optical galaxies in the farinfrared/submillimeter, but nevertheless, more can be done. Though the UDS is quite deep, deeper and/or wider fields and new catalogs are now coming online (e.g., Muzzin et al. 2013b; Ilbert et al. 2013; Viero et al. 2013a). That, combined with future ancillary data over large areas and to longer wavelengths, and with deeper near-infrared imaging and $24 \mu \mathrm{m}$ catalogs, should allow us to reach the coveted goal of resolving the full cosmic background.

The authors warmly thank Duncan Hanson, Phil Korngut, Zak Staniszewski, and Yoshihiro Ueda. We also thank the anonymous referee, whose careful comments greatly improved this paper. Much credit belongs to C. Barth Netterfield and Enzo Pascale for inspiring the simultaneous stacking algorithm, and 
to whom we are thankful. SPIRE has been developed by a consortium of institutes led by Cardiff Univ. (UK) and including: Univ. Lethbridge (Canada); NAOC (China); CEA, LAM (France); IFSI, Univ. Padua (Italy); IAC (Spain); Stockholm Observatory (Sweden); Imperial College London, RAL, UCLMSSL, UKATC, Univ. Sussex (UK); and Caltech, JPL, NHSC, Univ. Colorado (USA). This development has been supported by national funding agencies: CSA (Canada); NAOC (China); CEA, CNES, CNRS (France); ASI (Italy); MCINN (Spain); SNSB (Sweden); STFC, UKSA (UK); and NASA (USA).

\section{APPENDIX}

In this section we present tables corresponding to figures in the text. Tables 2 and 3 are the flux densities of star-forming and quiescent galaxies, respectively, in bins of stellar mass and redshift, as shown in Figure 5. Stacked intensities $\left.\nu\right|_{\nu}$, as shown in Figure 7, are tabulated in bins of redshift in Table 6, and stellar mass for star-forming and quiescent galaxies in Tables 7 and 8, respectively.

Table 2

Stacked Flux Densities of Star-forming Galaxies

\begin{tabular}{|c|c|c|c|c|c|}
\hline $\begin{array}{l}\lambda \\
(\mu \mathrm{m})\end{array}$ & $\log \left(M / M_{\odot}\right)=9.0-10.0$ & $\log \left(M / M_{\odot}\right)=10.0-11.0$ & $\begin{array}{c}z=0.0-0.5 \\
\log \left(M / M_{\odot}\right)=11.0-12.0\end{array}$ & $\log \left(M / M_{\odot}\right)=9.0-9.5$ & $\log \left(M / M_{\odot}\right)=9.5-10.0$ \\
\hline 24 & $(6.7 \pm 0.7) \times 10^{-2}$ & $(2.1 \pm 0.2) \times 10^{-1}$ & $(4.8 \pm 0.3) \times 10^{-1}$ & $(3.9 \pm 0.5) \times 10^{-1}$ & $(6.8 \pm 0.0) \times 10^{-1}$ \\
\hline 70 & $(7.0 \pm 1.3) \times 10^{-1}$ & $(2.4 \pm 0.2) \times 10^{0}$ & $(4.8 \pm 0.5) \times 10^{0}$ & $(3.8 \pm 0.8) \times 10^{0}$ & $(1.4 \pm 28.0) \times 10^{1}$ \\
\hline 100 & $(1.8 \pm 0.1) \times 10^{0}$ & $(5.8 \pm 0.4) \times 10^{0}$ & $(1.3 \pm 0.1) \times 10^{1}$ & $(1.1 \pm 0.1) \times 10^{1}$ & $(2.6 \pm 40.0) \times 10^{1}$ \\
\hline 160 & $(2.4 \pm 0.7) \times 10^{0}$ & $(7.2 \pm 1.0) \times 10^{0}$ & $(1.7 \pm 0.2) \times 10^{1}$ & $(1.8 \pm 0.3) \times 10^{1}$ & $(3.9 \pm 36.0) \times 10^{1}$ \\
\hline 250 & $(1.8 \pm 0.2) \times 10^{0}$ & $(5.5 \pm 0.4) \times 10^{0}$ & $(1.3 \pm 0.1) \times 10^{1}$ & $(1.5 \pm 0.1) \times 10^{1}$ & $(3.5 \pm 0.2) \times 10^{1}$ \\
\hline 350 & $(1.1 \pm 0.2) \times 10^{0}$ & $(3.1 \pm 0.3) \times 10^{0}$ & $(7.3 \pm 0.6) \times 10^{0}$ & $(9.4 \pm 1.1) \times 10^{0}$ & $(1.5 \pm 0.2) \times 10^{1}$ \\
\hline 500 & $(4.6 \pm 1.9) \times 10^{-1}$ & $(1.6 \pm 0.3) \times 10^{0}$ & $(3.6 \pm 0.5) \times 10^{0}$ & $(4.3 \pm 1.0) \times 10^{0}$ & $(2.1 \pm 1.7) \times 10^{0}$ \\
\hline 1100 & - & $(9.2 \pm 4.9) \times 10^{-2}$ & $(1.6 \pm 0.7) \times 10^{-1}$ & $(4.3 \pm 1.5) \times 10^{-1}$ & $(3.2 \pm 0.7) \times 10^{-1}$ \\
\hline $\begin{array}{l}\lambda \\
(\mu \mathrm{m}) \\
\end{array}$ & $\log \left(M / M_{\odot}\right)=9.0-10.0$ & $\log \left(M / M_{\odot}\right)=10.0-11.0$ & $\begin{array}{c}z=0.5-1.0 \\
\log \left(M / M_{\odot}\right)=11.0-12.0\end{array}$ & $\log \left(M / M_{\odot}\right)=9.0-9.5$ & $\log \left(M / M_{\odot}\right)=9.5-10.0$ \\
\hline 24 & $(1.4 \pm 0.1) \times 10^{-2}$ & $(8.5 \pm 0.4) \times 10^{-2}$ & $(2.0 \pm 0.1) \times 10^{-1}$ & $(3.6 \pm 0.1) \times 10^{-1}$ & $(2.1 \pm 0.3) \times 10^{-1}$ \\
\hline 70 & - & $(6.8 \pm 0.7) \times 10^{-1}$ & $(1.1 \pm 0.1) \times 10^{0}$ & $(2.0 \pm 0.2) \times 10^{0}$ & $(1.0 \pm 0.5) \times 10^{0}$ \\
\hline 100 & $(3.7 \pm 0.4) \times 10^{-1}$ & $(1.8 \pm 0.1) \times 10^{0}$ & $(4.0 \pm 0.1) \times 10^{0}$ & $(6.1 \pm 0.3) \times 10^{0}$ & $(2.3 \pm 0.8) \times 10^{0}$ \\
\hline 160 & $(4.8 \pm 3.0) \times 10^{-1}$ & $(2.7 \pm 0.4) \times 10^{0}$ & $(6.4 \pm 0.7) \times 10^{0}$ & $(1.0 \pm 0.1) \times 10^{1}$ & $(7.6 \pm 3.0) \times 10^{0}$ \\
\hline 250 & $(5.3 \pm 0.8) \times 10^{-1}$ & $(2.7 \pm 0.1) \times 10^{0}$ & $(6.7 \pm 0.3) \times 10^{0}$ & $(1.1 \pm 0.1) \times 10^{1}$ & $(8.9 \pm 1.4) \times 10^{0}$ \\
\hline 350 & $(4.1 \pm 0.9) \times 10^{-1}$ & $(1.8 \pm 0.1) \times 10^{0}$ & $(4.8 \pm 0.3) \times 10^{0}$ & $(8.6 \pm 0.5) \times 10^{0}$ & $(7.2 \pm 1.2) \times 10^{0}$ \\
\hline 500 & $(2.5 \pm 0.8) \times 10^{-1}$ & $(6.7 \pm 1.2) \times 10^{-1}$ & $(2.1 \pm 0.2) \times 10^{0}$ & $(4.8 \pm 0.4) \times 10^{0}$ & $(3.7 \pm 0.9) \times 10^{0}$ \\
\hline 1100 & - & $(1.3 \pm 2.0) \times 10^{-2}$ & $(1.7 \pm 0.3) \times 10^{-1}$ & $(2.9 \pm 0.7) \times 10^{-1}$ & $(5.2 \pm 1.7) \times 10^{-1}$ \\
\hline $\begin{array}{l}\lambda \\
(\mu \mathrm{m})\end{array}$ & $\log \left(M / M_{\odot}\right)=9.0-10.0$ & $\log \left(M / M_{\odot}\right)=10.0-11.0$ & $\begin{array}{c}z=1.0-1.5 \\
\log \left(M / M_{\odot}\right)=11.0-12.0\end{array}$ & $\log \left(M / M_{\odot}\right)=9.0-9.5$ & $\log \left(M / M_{\odot}\right)=9.5-10.0$ \\
\hline 24 & - & $(2.7 \pm 0.2) \times 10^{-2}$ & $(9.2 \pm 0.5) \times 10^{-2}$ & $(1.6 \pm 0.1) \times 10^{-1}$ & $(2.7 \pm 0.2) \times 10^{-1}$ \\
\hline 70 & - & $(4.7 \pm 5.3) \times 10^{-2}$ & $(4.9 \pm 0.8) \times 10^{-1}$ & $(1.1 \pm 0.1) \times 10^{0}$ & $(1.5 \pm 0.3) \times 10^{0}$ \\
\hline 100 & $(6.5 \pm 3.6) \times 10^{-2}$ & $(6.3 \pm 0.5) \times 10^{-1}$ & $(1.9 \pm 0.1) \times 10^{0}$ & $(3.5 \pm 0.1) \times 10^{0}$ & $(4.0 \pm 0.4) \times 10^{0}$ \\
\hline 160 & - & $(8.6 \pm 3.5) \times 10^{-1}$ & $(3.5 \pm 0.5) \times 10^{0}$ & $(6.8 \pm 0.8) \times 10^{0}$ & $(1.0 \pm 0.2) \times 10^{1}$ \\
\hline 250 & $(5.5 \pm 8.1) \times 10^{-2}$ & $(1.4 \pm 0.1) \times 10^{0}$ & $(4.2 \pm 0.2) \times 10^{0}$ & $(8.8 \pm 0.4) \times 10^{0}$ & $(1.4 \pm 0.1) \times 10^{1}$ \\
\hline 350 & - & $(1.3 \pm 0.1) \times 10^{0}$ & $(3.7 \pm 0.2) \times 10^{0}$ & $(8.0 \pm 0.4) \times 10^{0}$ & $(1.2 \pm 0.1) \times 10^{1}$ \\
\hline 500 & - & $(5.8 \pm 1.0) \times 10^{-1}$ & $(2.0 \pm 0.2) \times 10^{0}$ & $(4.5 \pm 0.3) \times 10^{0}$ & $(7.4 \pm 0.8) \times 10^{0}$ \\
\hline 1100 & - & $(2.2 \pm 1.8) \times 10^{-2}$ & $(1.3 \pm 0.3) \times 10^{-1}$ & $(4.4 \pm 0.5) \times 10^{-1}$ & $(3.7 \pm 1.0) \times 10^{-1}$ \\
\hline $\begin{array}{l}\lambda \\
(\mu \mathrm{m})\end{array}$ & $\log \left(M / M_{\odot}\right)=9.0-10.0$ & $\log \left(M / M_{\odot}\right)=10.0-11.0$ & $\begin{array}{c}z=1.5-2.0 \\
\log \left(M / M_{\odot}\right)=11.0-12.0\end{array}$ & $\log \left(M / M_{\odot}\right)=9.0-9.5$ & $\log \left(M / M_{\odot}\right)=9.5-10.0$ \\
\hline 24 & - & $(1.7 \pm 0.1) \times 10^{-2}$ & $(8.7 \pm 0.4) \times 10^{-2}$ & $(1.7 \pm 0.1) \times 10^{-1}$ & $(3.0 \pm 0.3) \times 10^{-1}$ \\
\hline 70 & - & - & $(2.7 \pm 0.9) \times 10^{-1}$ & $(3.9 \pm 1.3) \times 10^{-1}$ & $(1.6 \pm 0.2) \times 10^{0}$ \\
\hline 100 & $(1.4 \pm 0.4) \times 10^{-1}$ & $(2.4 \pm 0.6) \times 10^{-1}$ & $(1.3 \pm 0.1) \times 10^{0}$ & $(2.1 \pm 0.1) \times 10^{0}$ & $(3.8 \pm 0.3) \times 10^{0}$ \\
\hline 160 & - & $(5.0 \pm 4.4) \times 10^{-1}$ & $(2.3 \pm 0.6) \times 10^{0}$ & $(4.1 \pm 0.9) \times 10^{0}$ & $(7.5 \pm 1.8) \times 10^{0}$ \\
\hline 250 & - & $(8.6 \pm 1.3) \times 10^{-1}$ & $(3.1 \pm 0.2) \times 10^{0}$ & $(6.5 \pm 0.4) \times 10^{0}$ & $(1.3 \pm 0.1) \times 10^{1}$ \\
\hline 350 & - & $(8.6 \pm 1.4) \times 10^{-1}$ & $(3.0 \pm 0.2) \times 10^{0}$ & $(6.4 \pm 0.4) \times 10^{0}$ & $(1.3 \pm 0.1) \times 10^{1}$ \\
\hline 500 & - & $(6.0 \pm 1.3) \times 10^{-1}$ & $(1.9 \pm 0.2) \times 10^{0}$ & $(4.5 \pm 0.3) \times 10^{0}$ & $(8.9 \pm 0.7) \times 10^{0}$ \\
\hline 1100 & - & $(1.4 \pm 2.2) \times 10^{-2}$ & $(1.4 \pm 0.4) \times 10^{-1}$ & $(3.4 \pm 0.6) \times 10^{-1}$ & $(9.6 \pm 1.1) \times 10^{-1}$ \\
\hline $\begin{array}{l}\lambda \\
(\mu \mathrm{m})\end{array}$ & $\log \left(M / M_{\odot}\right)=9.0-10.0$ & $\log \left(M / M_{\odot}\right)=10.0-11.0$ & $\begin{array}{c}z=2.0-2.5 \\
\log \left(M / M_{\odot}\right)=11.0-12.0\end{array}$ & $\log \left(M / M_{\odot}\right)=9.0-9.5$ & $\log \left(M / M_{\odot}\right)=9.5-10.0$ \\
\hline 24 & - & $(1.1 \pm 1.6) \times 10^{-3}$ & $(5.2 \pm 0.4) \times 10^{-2}$ & $(1.3 \pm 0.1) \times 10^{-1}$ & $(2.7 \pm 0.1) \times 10^{-1}$ \\
\hline 70 & - & - & $(9.4 \pm 9.6) \times 10^{-2}$ & $(4.1 \pm 1.3) \times 10^{-1}$ & $(1.3 \pm 0.2) \times 10^{0}$ \\
\hline 100 & $(2.1 \pm 0.8) \times 10^{-1}$ & - & $(6.0 \pm 0.9) \times 10^{-1}$ & $(1.3 \pm 0.1) \times 10^{0}$ & $(2.7 \pm 0.2) \times 10^{0}$ \\
\hline 160 & - & - & $(1.3 \pm 0.7) \times 10^{0}$ & $(3.5 \pm 1.0) \times 10^{0}$ & $(6.8 \pm 1.4) \times 10^{0}$ \\
\hline 250 & - & $(7.5 \pm 15.0) \times 10^{-2}$ & $(2.0 \pm 0.2) \times 10^{0}$ & $(5.2 \pm 0.3) \times 10^{0}$ & $(1.2 \pm 0.1) \times 10^{1}$ \\
\hline 350 & - & $(2.8 \pm 1.6) \times 10^{-1}$ & $(2.3 \pm 0.2) \times 10^{0}$ & $(6.0 \pm 0.4) \times 10^{0}$ & $(1.3 \pm 0.1) \times 10^{1}$ \\
\hline 500 & - & $(3.5 \pm 1.7) \times 10^{-1}$ & $(2.0 \pm 0.2) \times 10^{0}$ & $(4.5 \pm 0.3) \times 10^{0}$ & $(1.0 \pm 0.1) \times 10^{1}$ \\
\hline 1100 & - & $(1.7 \pm 33.0) \times 10^{-3}$ & $(1.4 \pm 0.4) \times 10^{-1}$ & $(4.2 \pm 0.7) \times 10^{-1}$ & $(1.2 \pm 0.1) \times 10^{0}$ \\
\hline
\end{tabular}




\begin{tabular}{|c|c|c|c|c|c|}
\hline \multicolumn{6}{|c|}{$\begin{array}{c}\text { Table } 2 \\
\text { (Continued) }\end{array}$} \\
\hline $\begin{array}{l}\lambda \\
(\mu \mathrm{m})\end{array}$ & $\log \left(M / M_{\odot}\right)=9.0-10.0$ & $\log \left(M / M_{\odot}\right)=10.0-11.0$ & $\begin{array}{c}z=2.5-3.0 \\
\log \left(M / M_{\odot}\right)=11.0-12.0\end{array}$ & $\log \left(M / M_{\odot}\right)=9.0-9.5$ & $\log \left(M / M_{\odot}\right)=9.5-10.0$ \\
\hline 24 & - & $(9.3 \pm 16.0) \times 10^{-4}$ & $(2.0 \pm 0.2) \times 10^{-2}$ & $(7.4 \pm 0.4) \times 10^{-2}$ & $(2.1 \pm 0.2) \times 10^{-1}$ \\
\hline 70 & - & - & $(1.4 \pm 1.0) \times 10^{-1}$ & $(2.1 \pm 1.4) \times 10^{-1}$ & $(3.2 \pm 2.2) \times 10^{-1}$ \\
\hline 100 & $(1.5 \pm 110.0) \times 10^{-3}$ & $(2.1 \pm 0.7) \times 10^{-1}$ & $(4.2 \pm 0.9) \times 10^{-1}$ & $(1.1 \pm 0.1) \times 10^{0}$ & $(3.0 \pm 0.2) \times 10^{0}$ \\
\hline 160 & - & $(1.6 \pm 5.8) \times 10^{-1}$ & $(7.8 \pm 6.5) \times 10^{-1}$ & $(2.7 \pm 1.0) \times 10^{0}$ & $(7.2 \pm 1.4) \times 10^{0}$ \\
\hline 250 & - & $(2.9 \pm 1.5) \times 10^{-1}$ & $(1.4 \pm 0.2) \times 10^{0}$ & $(3.7 \pm 0.3) \times 10^{0}$ & $(1.1 \pm 0.1) \times 10^{1}$ \\
\hline 350 & - & $(4.1 \pm 1.7) \times 10^{-1}$ & $(1.6 \pm 0.2) \times 10^{0}$ & $(4.7 \pm 0.3) \times 10^{0}$ & $(1.4 \pm 0.1) \times 10^{1}$ \\
\hline 500 & - & $(3.0 \pm 1.7) \times 10^{-1}$ & $(1.0 \pm 0.2) \times 10^{0}$ & $(3.7 \pm 0.3) \times 10^{0}$ & $(1.1 \pm 0.1) \times 10^{1}$ \\
\hline 1100 & - & $(4.0 \pm 3.7) \times 10^{-2}$ & $(1.5 \pm 0.4) \times 10^{-1}$ & $(5.4 \pm 0.7) \times 10^{-1}$ & $(1.8 \pm 0.1) \times 10^{0}$ \\
\hline $\begin{array}{l}\lambda \\
(\mu \mathrm{m})\end{array}$ & $\log \left(M / M_{\odot}\right)=9.0-10.0$ & $\log \left(M / M_{\odot}\right)=10.0-11.0$ & $\begin{array}{c}z=3.0-3.5 \\
\log \left(M / M_{\odot}\right)=11.0-12.0\end{array}$ & $\log \left(M / M_{\odot}\right)=9.0-9.5$ & $\log \left(M / M_{\odot}\right)=9.5-10.0$ \\
\hline 24 & - & - & $(1.1 \pm 0.2) \times 10^{-2}$ & $(6.1 \pm 0.5) \times 10^{-2}$ & $(1.7 \pm 0.1) \times 10^{-1}$ \\
\hline 70 & - & - & - & - & $(1.2 \pm 0.3) \times 10^{0}$ \\
\hline 100 & - & $(5.8 \pm 9.6) \times 10^{-2}$ & $(2.6 \pm 1.1) \times 10^{-1}$ & $(1.4 \pm 0.2) \times 10^{0}$ & $(2.8 \pm 0.3) \times 10^{0}$ \\
\hline 160 & - & - & $(6.4 \pm 7.9) \times 10^{-1}$ & $(2.8 \pm 1.4) \times 10^{0}$ & $(6.3 \pm 2.5) \times 10^{0}$ \\
\hline 250 & - & - & $(1.3 \pm 0.2) \times 10^{0}$ & $(5.1 \pm 0.4) \times 10^{0}$ & $(1.2 \pm 0.1) \times 10^{1}$ \\
\hline 350 & - & $(8.5 \pm 25.0) \times 10^{-2}$ & $(1.8 \pm 0.3) \times 10^{0}$ & $(7.1 \pm 0.5) \times 10^{0}$ & $(1.5 \pm 0.1) \times 10^{1}$ \\
\hline 500 & $(3.1 \pm 47.0) \times 10^{-2}$ & $(3.1 \pm 2.6) \times 10^{-1}$ & $(1.8 \pm 0.3) \times 10^{0}$ & $(6.6 \pm 0.5) \times 10^{0}$ & $(1.3 \pm 0.1) \times 10^{1}$ \\
\hline 1100 & - & - & $(1.9 \pm 0.5) \times 10^{-1}$ & $(7.9 \pm 1.2) \times 10^{-1}$ & $(2.0 \pm 0.2) \times 10^{0}$ \\
\hline $\begin{array}{l}\lambda \\
(\mu \mathrm{m})\end{array}$ & $\log \left(M / M_{\odot}\right)=9.0-10.0$ & $\log \left(M / M_{\odot}\right)=10.0-11.0$ & $\begin{array}{c}z=3.5-4.0 \\
\log \left(M / M_{\odot}\right)=11.0-12.0\end{array}$ & $\log \left(M / M_{\odot}\right)=9.0-9.5$ & $\log \left(M / M_{\odot}\right)=9.5-10.0$ \\
\hline 24 & $(6.4 \pm 0.2) \times 10^{-2}$ & - & - & $(3.1 \pm 1.0) \times 10^{-2}$ & $(1.1 \pm 0.3) \times 10^{-1}$ \\
\hline 70 & $(7.8 \pm 1000.0) \times 10^{-1}$ & - & - & $(3.7 \pm 4.5) \times 10^{-1}$ & $(3.3 \pm 8.7) \times 10^{-1}$ \\
\hline 100 & $(2.3 \pm 1500.0) \times 10^{-1}$ & $(3.7 \pm 27.0) \times 10^{-2}$ & $(8.2 \pm 22.0) \times 10^{-2}$ & $(4.7 \pm 3.7) \times 10^{-1}$ & $(2.9 \pm 7.1) \times 10^{-1}$ \\
\hline 160 & $(3.8 \pm 1400.0) \times 10^{-1}$ & - & - & $(2.5 \pm 3.1) \times 10^{0}$ & $(5.4 \pm 6.6) \times 10^{0}$ \\
\hline 250 & $(7.0 \pm 0.6) \times 10^{0}$ & - & $(5.6 \pm 5.3) \times 10^{-1}$ & $(3.2 \pm 1.0) \times 10^{0}$ & $(1.1 \pm 0.2) \times 10^{1}$ \\
\hline 350 & $(7.0 \pm 0.6) \times 10^{0}$ & - & $(7.1 \pm 6.3) \times 10^{-1}$ & $(3.8 \pm 1.2) \times 10^{0}$ & $(1.6 \pm 0.3) \times 10^{1}$ \\
\hline 500 & $(6.2 \pm 0.7) \times 10^{0}$ & $(9.9 \pm 7.1) \times 10^{-1}$ & $(1.0 \pm 0.6) \times 10^{0}$ & $(3.3 \pm 1.1) \times 10^{0}$ & $(1.5 \pm 0.2) \times 10^{1}$ \\
\hline 1100 & $(9.0 \pm 0.5) \times 10^{-1}$ & $(1.2 \pm 1.4) \times 10^{-1}$ & $(2.1 \pm 1.3) \times 10^{-1}$ & $(5.8 \pm 2.5) \times 10^{-1}$ & $(2.4 \pm 0.0) \times 10^{0}$ \\
\hline
\end{tabular}

Notes. Stacked flux densities in mJy. Errors estimated with a extended bootstrap technique described in Section 3.4. Blank spaces represent where the algorithm fails to adequately converge. Flux densities, converted to $v I_{\nu}$, are shown in Figure 7.

Table 3

Stacked Flux Densities of Quiescent Galaxies

\begin{tabular}{|c|c|c|c|}
\hline $\begin{array}{l}\lambda \\
(\mu \mathrm{m})\end{array}$ & $\log \left(M / M_{\odot}\right)=9.0-10.0$ & $\begin{array}{c}z=0.0-0.5 \\
\log \left(M / M_{\odot}\right)=10.0-11.0\end{array}$ & $\log \left(M / M_{\odot}\right)=11.0-12.0$ \\
\hline$\overline{24}$ & $(1.4 \pm 0.5) \times 10^{-2}$ & $(2.8 \pm 0.4) \times 10^{-2}$ & $(1.0 \pm 0.2) \times 10^{-1}$ \\
\hline 70 & $(8.3 \pm 20.0) \times 10^{-2}$ & $(1.5 \pm 1.9) \times 10^{-1}$ & - \\
\hline 100 & $(8.2 \pm 1.7) \times 10^{-1}$ & $(7.4 \pm 1.5) \times 10^{-1}$ & $(1.7 \pm 0.6) \times 10^{0}$ \\
\hline 160 & $(4.7 \pm 13.0) \times 10^{-1}$ & $(8.5 \pm 12.0) \times 10^{-1}$ & $(4.0 \pm 5.5) \times 10^{0}$ \\
\hline 250 & - & $(5.6 \pm 2.8) \times 10^{-1}$ & $(2.4 \pm 1.1) \times 10^{0}$ \\
\hline 350 & - & $(2.6 \pm 3.0) \times 10^{-1}$ & $(1.3 \pm 1.3) \times 10^{0}$ \\
\hline 500 & - & - & $(7.7 \pm 13.0) \times 10^{-1}$ \\
\hline 1100 & - & - & - \\
\hline$\lambda$ & & $z=0.5-1.0$ & \\
\hline$\underline{(\mu \mathrm{m})}$ & $\log \left(M / M_{\odot}\right)=9.0-10.0$ & $\log \left(M / M_{\odot}\right)=10.0-11.0$ & $\log \left(M / M_{\odot}\right)=11.0-12.0$ \\
\hline 24 & - & $(1.4 \pm 0.2) \times 10^{-2}$ & $(2.4 \pm 0.7) \times 10^{-2}$ \\
\hline 70 & $(6.5 \pm 11.0) \times 10^{-2}$ & - & - \\
\hline 100 & $(7.6 \pm 8.5) \times 10^{-2}$ & $(3.3 \pm 0.7) \times 10^{-1}$ & $(3.8 \pm 2.2) \times 10^{-1}$ \\
\hline 160 & $(4.0 \pm 6.3) \times 10^{-1}$ & $(5.7 \pm 51.0) \times 10^{-2}$ & - \\
\hline 250 & $(3.8 \pm 1.9) \times 10^{-1}$ & - & $(4.7 \pm 5.1) \times 10^{-1}$ \\
\hline 350 & $(5.7 \pm 2.1) \times 10^{-1}$ & - & $(2.1 \pm 6.0) \times 10^{-1}$ \\
\hline 500 & $(3.6 \pm 1.9) \times 10^{-1}$ & - & - \\
\hline 1100 & $(4.5 \pm 3.6) \times 10^{-2}$ & - & $(6.0 \pm 11.0) \times 10^{-2}$ \\
\hline$\lambda$ & & $z=1.0-1.5$ & \\
\hline$(\mu \mathrm{m})$ & $\log \left(M / M_{\odot}\right)=9.0-10.0$ & $\log \left(M / M_{\odot}\right)=10.0-11.0$ & $\log \left(M / M_{\odot}\right)=11.0-12.0$ \\
\hline 24 & - & $(5.9 \pm 1.7) \times 10^{-3}$ & $(2.1 \pm 0.6) \times 10^{-2}$ \\
\hline 70 & - & - & $(2.4 \pm 2.2) \times 10^{-1}$ \\
\hline 100 & - & - & - \\
\hline 160 & - & $(3.2 \pm 4.7) \times 10^{-1}$ & $(6.1 \pm 14.0) \times 10^{-1}$ \\
\hline 250 & - & $(2.4 \pm 1.4) \times 10^{-1}$ & $(1.1 \pm 0.4) \times 10^{0}$ \\
\hline
\end{tabular}


Table 3

(Continued)

\begin{tabular}{|c|c|c|c|}
\hline $\begin{array}{l}\lambda \\
(\mu \mathrm{m})\end{array}$ & $\log \left(M / M_{\odot}\right)=9.0-10.0$ & $\begin{array}{c}z=1.0-1.5 \\
\log \left(M / M_{\odot}\right)=10.0-11.0\end{array}$ & $\log \left(M / M_{\odot}\right)=11.0-12.0$ \\
\hline 350 & - & $(3.1 \pm 1.7) \times 10^{-1}$ & $(1.7 \pm 0.5) \times 10^{0}$ \\
\hline 500 & - & $(1.2 \pm 1.6) \times 10^{-1}$ & $(1.4 \pm 0.5) \times 10^{0}$ \\
\hline 1100 & - & - & - \\
\hline $\begin{array}{l}\lambda \\
(\mu \mathrm{m})\end{array}$ & $\log \left(M / M_{\odot}\right)=9.0-10.0$ & $\begin{array}{c}z=1.5-2.0 \\
\log \left(M / M_{\odot}\right)=10.0-11.0\end{array}$ & $\log \left(M / M_{\odot}\right)=11.0-12.0$ \\
\hline 24 & - & $(1.3 \pm 1.8) \times 10^{-3}$ & $(1.1 \pm 0.7) \times 10^{-2}$ \\
\hline 70 & - & - & - \\
\hline 100 & - & - & $(2.1 \pm 24.0) \times 10^{-2}$ \\
\hline 160 & - & - & $(9.2 \pm 22.0) \times 10^{-1}$ \\
\hline 250 & - & $(1.6 \pm 1.9) \times 10^{-1}$ & - \\
\hline 350 & $(2.3 \pm 3.4) \times 10^{-1}$ & $(3.9 \pm 2.4) \times 10^{-1}$ & $(4.7 \pm 7.4) \times 10^{-1}$ \\
\hline 500 & $(3.8 \pm 3.6) \times 10^{-1}$ & $(3.7 \pm 2.3) \times 10^{-1}$ & $(4.9 \pm 7.6) \times 10^{-1}$ \\
\hline 1100 & - & - & $(1.6 \pm 1.2) \times 10^{-1}$ \\
\hline$\lambda$ & & $z=2.0-2.5$ & \\
\hline$(\mu \mathrm{m})$ & $\log \left(M / M_{\odot}\right)=9.0-10.0$ & $\log \left(M / M_{\odot}\right)=10.0-11.0$ & $\log \left(M / M_{\odot}\right)=11.0-12.0$ \\
\hline 24 & - & $(8.7 \pm 3.4) \times 10^{-3}$ & $(5.7 \pm 0.7) \times 10^{-2}$ \\
\hline 70 & $(2.5 \pm 32.0) \times 10^{-2}$ & - & $(3.1 \pm 2.8) \times 10^{-1}$ \\
\hline 100 & - & - & $(1.4 \pm 0.2) \times 10^{0}$ \\
\hline 160 & $(3.7 \pm 2.2) \times 10^{0}$ & $(8.3 \pm 12.0) \times 10^{-1}$ & $(4.1 \pm 1.9) \times 10^{0}$ \\
\hline 250 & $(9.3 \pm 49.0) \times 10^{-2}$ & $(7.1 \pm 3.1) \times 10^{-1}$ & $(4.1 \pm 0.6) \times 10^{0}$ \\
\hline 350 & $(1.7 \pm 0.6) \times 10^{0}$ & $(1.0 \pm 0.4) \times 10^{0}$ & $(4.6 \pm 0.7) \times 10^{0}$ \\
\hline 500 & $(1.9 \pm 0.6) \times 10^{0}$ & $(8.2 \pm 3.5) \times 10^{-1}$ & $(3.6 \pm 0.6) \times 10^{0}$ \\
\hline 1100 & - & - & $(2.3 \pm 1.1) \times 10^{-1}$ \\
\hline$\lambda$ & & $z=2.5-3.0$ & \\
\hline$(\mu \mathrm{m})$ & $\log \left(M / M_{\odot}\right)=9.0-10.0$ & $\log \left(M / M_{\odot}\right)=10.0-11.0$ & $\log \left(M / M_{\odot}\right)=11.0-12.0$ \\
\hline 24 & - & $(2.0 \pm 0.3) \times 10^{-2}$ & $(8.2 \pm 0.5) \times 10^{-2}$ \\
\hline 70 & - & - & $(8.0 \pm 24.0) \times 10^{-2}$ \\
\hline 100 & - & $(2.7 \pm 1.5) \times 10^{-1}$ & $(7.7 \pm 1.7) \times 10^{-1}$ \\
\hline 160 & - & $(5.1 \pm 15.0) \times 10^{-1}$ & $(1.5 \pm 1.6) \times 10^{0}$ \\
\hline 250 & - & $(1.1 \pm 0.3) \times 10^{0}$ & $(3.8 \pm 0.4) \times 10^{0}$ \\
\hline 350 & - & $(1.8 \pm 0.4) \times 10^{0}$ & $(4.1 \pm 0.6) \times 10^{0}$ \\
\hline 500 & - & $(1.8 \pm 0.4) \times 10^{0}$ & $(3.2 \pm 0.5) \times 10^{0}$ \\
\hline 1100 & - & $(2.0 \pm 0.8) \times 10^{-1}$ & $(3.3 \pm 1.1) \times 10^{-1}$ \\
\hline$\lambda$ & & $z=3.0-3.5$ & \\
\hline$(\mu \mathrm{m})$ & $\log \left(M / M_{\odot}\right)=9.0-10.0$ & $\log \left(M / M_{\odot}\right)=10.0-11.0$ & $\log \left(M / M_{\odot}\right)=11.0-12.0$ \\
\hline 24 & - & $(2.0 \pm 0.4) \times 10^{-2}$ & $(8.5 \pm 0.7) \times 10^{-2}$ \\
\hline 70 & $(2.4 \pm 200.0) \times 10^{0}$ & - & - \\
\hline 100 & $(4.2 \pm 280.0) \times 10^{0}$ & $(7.1 \pm 2.2) \times 10^{-1}$ & $(1.7 \pm 0.2) \times 10^{0}$ \\
\hline 160 & - & $(2.6 \pm 16.0) \times 10^{-1}$ & $(3.2 \pm 1.9) \times 10^{0}$ \\
\hline 250 & $(2.3 \pm 1.1) \times 10^{0}$ & $(9.9 \pm 4.4) \times 10^{-1}$ & $(5.7 \pm 0.6) \times 10^{0}$ \\
\hline 350 & $(2.5 \pm 1.1) \times 10^{0}$ & $(1.6 \pm 0.6) \times 10^{0}$ & $(6.2 \pm 0.8) \times 10^{0}$ \\
\hline 500 & $(4.8 \pm 1.3) \times 10^{0}$ & $(1.2 \pm 0.5) \times 10^{0}$ & $(4.8 \pm 0.8) \times 10^{0}$ \\
\hline 1100 & - & $(2.3 \pm 1.1) \times 10^{-1}$ & $(4.2 \pm 1.4) \times 10^{-1}$ \\
\hline$\lambda$ & & $z=3.5-4.0$ & \\
\hline$(\mu \mathrm{m})$ & $\log \left(M / M_{\odot}\right)=9.0-10.0$ & $\log \left(M / M_{\odot}\right)=10.0-11.0$ & $\log \left(M / M_{\odot}\right)=11.0-12.0$ \\
\hline 24 & - & $(4.2 \pm 5.1) \times 10^{-3}$ & $(5.4 \pm 1.1) \times 10^{-2}$ \\
\hline 70 & - & $(1.6 \pm 4.6) \times 10^{-1}$ & - \\
\hline 100 & - & - & $(6.6 \pm 4.8) \times 10^{-1}$ \\
\hline 160 & - & $(1.5 \pm 290.0) \times 10^{-2}$ & $(5.1 \pm 36.0) \times 10^{-1}$ \\
\hline 250 & - & $(3.7 \pm 0.8) \times 10^{0}$ & $(3.8 \pm 1.1) \times 10^{0}$ \\
\hline 350 & - & $(4.7 \pm 1.1) \times 10^{0}$ & $(5.9 \pm 1.4) \times 10^{0}$ \\
\hline 500 & - & $(3.2 \pm 1.1) \times 10^{0}$ & $(5.9 \pm 1.5) \times 10^{0}$ \\
\hline 1100 & - & $(4.3 \pm 3.4) \times 10^{-1}$ & $(1.7 \pm 0.4) \times 10^{0}$ \\
\hline
\end{tabular}

Notes. Stacked flux densities in mJy. Errors estimated with a extended bootstrap technique described in Section 3.4. Blank spaces represent where the algorithm fails to adequately converge. Flux densities, converted to $v I_{v}$, are shown in Figure 7. 
Table 4

Pearson Correlation Matrix for All Bands Under Analysis

\begin{tabular}{lcccccccc}
\hline \hline $\begin{array}{l}\text { Band } \\
(\mu \mathrm{m})\end{array}$ & 24 & 70 & 100 & 160 & 250 & 350 & 500 & 1100 \\
\hline 24 & 1 & 0.23 & 0.33 & 0.32 & 0.28 & 0.17 & 0.07 & 0.10 \\
70 & & 1 & 0.19 & 0.24 & 0.23 & 0.14 & 0.06 & 0.08 \\
100 & & & 1 & 0.28 & 0.21 & 0.11 & 0.04 & 0.05 \\
160 & & & & 1 & 0.35 & 0.23 & 0.10 & 0.13 \\
250 & & & & & 1 & 0.37 & 0.18 & 0.28 \\
350 & & & & & & 1 & 0.20 & 0.33 \\
500 & & & & & & & & 0.23 \\
1100 & & & & & & & & \\
\hline
\end{tabular}

Table 5

Total Stacked Intensities

\begin{tabular}{|c|c|c|c|c|c|}
\hline \multirow[b]{2}{*}{$\begin{array}{l}\text { Band } \\
(\mu \mathrm{m})\end{array}$} & \multicolumn{3}{|c|}{ This Work } & \multicolumn{2}{|c|}{ Absolute Measurements } \\
\hline & $\begin{array}{l}\text { Total Stacking } \\
\left(\mathrm{nW} \mathrm{m}^{-2} \mathrm{sr}^{-1}\right)\end{array}$ & $\begin{array}{l}\text { Completeness Corrected } \\
\left(\mathrm{nW} \mathrm{m}^{-2} \mathrm{sr}^{-1}\right)\end{array}$ & $\begin{array}{l}\text { Total Model SEDs } \\
\left(\mathrm{nW} \mathrm{m} \mathrm{m}^{-2} \mathrm{sr}^{-1}\right)\end{array}$ & $\begin{array}{l}\text { Absolute CIB } \\
\left(\mathrm{nW} \mathrm{m}^{-2} \mathrm{sr}^{-1}\right)\end{array}$ & Reference \\
\hline 24 & $1.84 \pm 0.05(64 \% \pm 1 \%)$ & $1.87 \pm 0.05(65 \% \pm 1 \%)$ & $1.99 \pm 0.46(69 \% \pm 15 \%)$ & $2.86 \pm 0.17$ & Béthermin et al. (2010) \\
\hline 70 & $3.31 \pm 0.20(50 \% \pm 3 \%)$ & $3.33 \pm 0.20(50 \% \pm 3 \%)$ & $5.17 \pm 1.20(78 \% \pm 18 \%)$ & $6.60 \pm 0.70$ & Béthermin et al. (2010) \\
\hline 100 & $8.74 \pm 0.38(69 \% \pm 3 \%)$ & $8.84 \pm 0.39(70 \% \pm 3 \%)$ & $7.35 \pm 1.72(58 \% \pm 13 \%)$ & $12.60 \pm 4.00$ & Berta et al. (2011) \\
\hline 160 & $9.43 \pm 0.63(69 \% \pm 4 \%)$ & $9.57 \pm 0.64(70 \% \pm 4 \%)$ & $10.66 \pm 2.50(78 \% \pm 18 \%)$ & $13.60 \pm 2.50$ & Berta et al. (2011) \\
\hline 250 & $7.00 \pm 0.34(67 \% \pm 3 \%)$ & $7.14 \pm 0.35(68 \% \pm 3 \%)$ & $8.39 \pm 1.83(80 \% \pm 17 \%)$ & $10.40 \pm 2.30$ & Lagache et al. (2000) \\
\hline 350 & $4.38 \pm 0.22(67 \% \pm 3 \%)$ & $4.50 \pm 0.23(69 \% \pm 3 \%)$ & $4.54 \pm 0.92(69 \% \pm 14 \%)$ & $6.50 \pm 1.60$ & Lagache et al. (2000) \\
\hline 500 & $1.84 \pm 0.10(70 \% \pm 3 \%)$ & $1.91 \pm 0.11(73 \% \pm 4 \%)$ & $1.69 \pm 0.32(65 \% \pm 12 \%)$ & $2.60 \pm 0.60$ & Lagache et al. (2000) \\
\hline 1100 & $0.06 \pm 0.01(34 \% \pm 2 \%)$ & $0.07 \pm 0.01(35 \% \pm 2 \%)$ & $0.09 \pm 0.02(45 \% \pm 8 \%)$ & $0.19 \pm 0.04$ & Lagache et al. (2000) \\
\hline
\end{tabular}

Notes. Errors estimated with a extended bootstrap technique described in Section 3.4. In parentheses are the percentages of the total CIB resolved, as measured at $24-160 \mu \mathrm{m}$ by MIPS; and at $250-1100 \mu \mathrm{m}$ by FIRAS.

Table 6

Stacked Intensities in Bins of Redshift

\begin{tabular}{|c|c|c|c|c|c|}
\hline $\begin{array}{l}\text { Band } \\
(\mu m)\end{array}$ & Type & $\begin{array}{c}z=0.0-1.0 \\
\left(\mathrm{nW} \mathrm{m}^{-2} \mathrm{sr}^{-1}\right)\end{array}$ & $\begin{array}{c}z=1.0-2.0 \\
\left(\mathrm{nW} \mathrm{m} \mathrm{m}^{-2} \mathrm{sr}^{-1}\right)\end{array}$ & $\begin{array}{c}z=2.0-3.0 \\
\left(\mathrm{nW} \mathrm{m} \mathrm{m}^{-2} \mathrm{sr}^{-1}\right)\end{array}$ & $\begin{array}{c}z=3.0-4.0 \\
\left(\mathrm{nW} \mathrm{m}^{-2} \mathrm{sr}^{-1}\right)\end{array}$ \\
\hline 24 & $\begin{array}{c}\text { Stack } \\
\text { CC } \\
\text { SED }\end{array}$ & $\begin{array}{l}9.2 \pm 0.2) \times 10^{-1}[50 \%] \\
(9.2 \pm 0.2) \times 10^{-1}[39 \%] \\
(1.0 \pm 0.4) \times 10^{0}[44 \%]\end{array}$ & $\begin{array}{l}(6.8 \pm 0.2) \times 10^{-1}[36 \%] \\
(7.3 \pm 0.3) \times 10^{-1}[31 \%] \\
(8.3 \pm 2.9) \times 10^{-1}[35 \%]\end{array}$ & $\begin{array}{l}(2.1 \pm 0.1) \times 10^{-1}[11 \%] \\
(3.6 \pm 0.4) \times 10^{-1}[15 \%] \\
(3.5 \pm 1.3) \times 10^{-1}[15 \%]\end{array}$ & $\begin{array}{l}(3.0 \pm 0.2) \times 10^{-2}[1 \%] \\
(3.0 \pm 0.5) \times 10^{-1}[13 \%] \\
(3.6 \pm 0.0) \times 10^{-1}[15 \%]\end{array}$ \\
\hline 70 & $\begin{array}{l}\text { Stack } \\
\text { CC } \\
\text { SED }\end{array}$ & $\begin{array}{l}(2.2 \pm 0.1) \times 10^{0}[66 \%] \\
(2.2 \pm 0.1) \times 10^{0}[62 \%] \\
(2.7 \pm 1.0) \times 10^{0}[76 \%]\end{array}$ & $\begin{array}{l}(8.7 \pm 1.1) \times 10^{-1}[26 \%] \\
(8.7 \pm 2.0) \times 10^{-1}[24 \%] \\
(2.1 \pm 0.7) \times 10^{0}[59 \%]\end{array}$ & $\begin{array}{l}(2.1 \pm 0.7) \times 10^{-1}[6 \%] \\
(4.3 \pm 8.0) \times 10^{-1}[12 \%] \\
(8.7 \pm 3.2) \times 10^{-1}[24 \%]\end{array}$ & $\begin{array}{l}(2.7 \pm 3.7) \times 10^{-2}[0 \%] \\
(5.0 \pm 130.0) \times 10^{-2}[1 \%] \\
(9.3 \pm 0.0) \times 10^{-1}[26 \%]\end{array}$ \\
\hline 100 & $\begin{array}{l}\text { Stack } \\
\text { CC } \\
\text { SED }\end{array}$ & $\begin{array}{l}(4.9 \pm 0.2) \times 10^{0}[56 \%] \\
(4.9 \pm 0.2) \times 10^{0}[44 \%] \\
(3.9 \pm 1.4) \times 10^{0}[35 \%]\end{array}$ & $\begin{array}{l}(3.0 \pm 0.1) \times 10^{0}[33 \%] \\
(3.2 \pm 0.2) \times 10^{0}[29 \%] \\
(3.0 \pm 1.0) \times 10^{0}[26 \%]\end{array}$ & $\begin{array}{l}(6.9 \pm 0.4) \times 10^{-1}[7 \%] \\
(1.2 \pm 0.4) \times 10^{0}[10 \%] \\
(1.2 \pm 0.5) \times 10^{0}[10 \%]\end{array}$ & $\begin{array}{l}(1.5 \pm 0.2) \times 10^{-1}[1 \%] \\
(1.7 \pm 0.6) \times 10^{0}[15 \%] \\
(1.3 \pm 0.0) \times 10^{0}[11 \%]\end{array}$ \\
\hline 160 & $\begin{array}{l}\text { Stack } \\
\text { CC } \\
\text { SED }\end{array}$ & $\begin{array}{l}(4.8 \pm 0.3) \times 10^{0}[51 \%] \\
(4.8 \pm 0.3) \times 10^{0}[37 \%] \\
(5.3 \pm 2.0) \times 10^{0}[41 \%]\end{array}$ & $\begin{array}{l}(3.4 \pm 0.3) \times 10^{0}[36 \%] \\
(3.7 \pm 0.6) \times 10^{0}[28 \%] \\
(4.6 \pm 1.6) \times 10^{0}[36 \%]\end{array}$ & $\begin{array}{l}(1.0 \pm 0.2) \times 10^{0}[10 \%] \\
(1.8 \pm 2.2) \times 10^{0}[13 \%] \\
(1.9 \pm 0.7) \times 10^{0}[14 \%]\end{array}$ & $\begin{array}{l}(1.8 \pm 0.9) \times 10^{-1}[1 \%] \\
(2.5 \pm 2.8) \times 10^{0}[19 \%] \\
(2.1 \pm 0.0) \times 10^{0}[16 \%]\end{array}$ \\
\hline 250 & $\begin{array}{l}\text { Stack } \\
\text { CC } \\
\text { SED }\end{array}$ & $\begin{array}{l}(2.7 \pm 0.1) \times 10^{0}[38 \%] \\
(2.7 \pm 0.1) \times 10^{0}[23 \%] \\
(2.9 \pm 1.1) \times 10^{0}[25 \%]\end{array}$ & $\begin{array}{l}(3.0 \pm 0.2) \times 10^{0}[43 \%] \\
(3.3 \pm 0.2) \times 10^{0}[29 \%] \\
(4.3 \pm 1.5) \times 10^{0}[37 \%]\end{array}$ & $\begin{array}{l}(1.0 \pm 0.1) \times 10^{0}[14 \%] \\
(1.9 \pm 0.4) \times 10^{0}[16 \%] \\
(2.6 \pm 0.9) \times 10^{0}[22 \%]\end{array}$ & $\begin{array}{l}(2.5 \pm 0.2) \times 10^{-1}[3 \%] \\
(3.5 \pm 0.5) \times 10^{0}[30 \%] \\
(3.1 \pm 0.0) \times 10^{0}[27 \%]\end{array}$ \\
\hline 350 & $\begin{array}{l}\text { Stack } \\
\text { CC } \\
\text { SED }\end{array}$ & $\begin{array}{l}(1.3 \pm 0.1) \times 10^{0}[29 \%] \\
(1.3 \pm 0.1) \times 10^{0}[14 \%] \\
(1.1 \pm 0.4) \times 10^{0}[12 \%]\end{array}$ & $\begin{array}{l}2.0 \pm 0.1) \times 10^{0}[45 \%] \\
(2.2 \pm 0.1) \times 10^{0}[24 \%] \\
(2.3 \pm 0.8) \times 10^{0}[26 \%]\end{array}$ & $\begin{array}{l}(8.8 \pm 0.4) \times 10^{-1}[20 \%] \\
(2.0 \pm 0.3) \times 10^{0}[22 \%] \\
(2.1 \pm 0.7) \times 10^{0}[23 \%]\end{array}$ & $\begin{array}{l}(2.4 \pm 0.2) \times 10^{-1}[5 \%] \\
(3.4 \pm 0.5) \times 10^{0}[38 \%] \\
(3.1 \pm 0.0) \times 10^{0}[35 \%]\end{array}$ \\
\hline 500 & $\begin{array}{l}\text { Stack } \\
\text { CC } \\
\text { SED }\end{array}$ & $\begin{array}{l}(4.1 \pm 0.3) \times 10^{-1}[21 \%] \\
(4.1 \pm 0.3) \times 10^{-1}[8 \%] \\
(3.3 \pm 1.3) \times 10^{-1}[6 \%]\end{array}$ & $\begin{array}{l}(8.0 \pm 0.5) \times 10^{-1}[43 \%] \\
(8.9 \pm 0.7) \times 10^{-1}[18 \%] \\
(8.2 \pm 2.7) \times 10^{-1}[17 \%]\end{array}$ & $\begin{array}{l}(4.8 \pm 0.3) \times 10^{-1}[25 \%] \\
(1.2 \pm 0.2) \times 10^{0}[25 \%] \\
(1.0 \pm 0.3) \times 10^{0}[21 \%]\end{array}$ & $\begin{array}{l}(1.6 \pm 0.1) \times 10^{-1}[8 \%] \\
(2.3 \pm 0.3) \times 10^{0}[47 \%] \\
(1.7 \pm 0.0) \times 10^{0}[36 \%]\end{array}$ \\
\hline 1100 & $\begin{array}{c}\text { Stack } \\
\text { CC } \\
\text { SED }\end{array}$ & $\begin{array}{l}(9.6 \pm 1.5) \times 10^{-3}[14 \%] \\
(9.6 \pm 1.5) \times 10^{-3}[5 \%] \\
(1.2 \pm 0.5) \times 10^{-2}[6 \%]\end{array}$ & $\begin{array}{l}(2.5 \pm 0.3) \times 10^{-2}[38 \%] \\
(2.6 \pm 0.4) \times 10^{-2}[14 \%] \\
(4.0 \pm 1.3) \times 10^{-2}[22 \%]\end{array}$ & $\begin{array}{l}(2.0 \pm 0.2) \times 10^{-2}[31 \%] \\
(3.5 \pm 1.7) \times 10^{-2}[20 \%] \\
(6.3 \pm 1.9) \times 10^{-2}[36 \%]\end{array}$ & $\begin{array}{l}(9.6 \pm 1.0) \times 10^{-3}[14 \%] \\
(1.0 \pm 0.3) \times 10^{-1}[59 \%] \\
(1.2 \pm 0.0) \times 10^{-1}[70 \%]\end{array}$ \\
\hline
\end{tabular}

Notes. Stacked intensities in units of $\mathrm{nW} \mathrm{m}^{-2} \mathrm{sr}^{-1}$. Uncorrected intensities are labeled "Stack," completeness-corrected values are labeled "CC," and the best-fit SED values are labeled "SED." Errors estimated with a Monte Carlo simulation described in Section 3.4. Blank spaces represent where the algorithm fails to adequately converge. 
Table 7

Stacked Intensities of Star-forming Galaxies in Bins of Stellar Mass

\begin{tabular}{|c|c|c|c|c|c|c|}
\hline $\begin{array}{l}\text { Band } \\
(\mu m)\end{array}$ & Type & $\begin{array}{c}\log \left(M / M_{\odot}\right)=9.0-9.5 \\
\left(\mathrm{nW} \mathrm{m}^{-2} \mathrm{sr}^{-1}\right)\end{array}$ & $\begin{array}{c}\log \left(M / M_{\odot}\right)=9.5-10.0 \\
\left(\mathrm{nW} \mathrm{m}^{-2} \mathrm{sr}^{-1}\right)\end{array}$ & $\begin{array}{c}\log \left(M / M_{\odot}\right)=10.0-10.5 \\
\left(\mathrm{nW} \mathrm{m}^{-2} \mathrm{sr}^{-1}\right)\end{array}$ & $\begin{array}{c}\log \left(M / M_{\odot}\right)=10.5-11.0 \\
\left(\mathrm{nW} \mathrm{m}^{-2} \mathrm{sr}^{-1}\right)\end{array}$ & $\begin{array}{c}\log \left(M / M_{\odot}\right)=11.0-12.0 \\
\left(\mathrm{nW} \mathrm{m}^{-2} \mathrm{sr}^{-1}\right)\end{array}$ \\
\hline \multirow[t]{3}{*}{24} & Stack & $(1.2 \pm 0.1) \times 10^{-1}[6 \%]$ & $(4.0 \pm 0.1) \times 10^{-1}[21 \%]$ & $(6.9 \pm 0.2) \times 10^{-1}[37 \%]$ & $(4.6 \pm 0.1) \times 10^{-1}[24 \%]$ & $(1.1 \pm 0.0) \times 10^{-1}[6 \%]$ \\
\hline & $\mathrm{CC}$ & $(1.2 \pm 0.1) \times 10^{-1}[5 \%]$ & $(4.8 \pm 0.4) \times 10^{-1}[20 \%]$ & $(1.1 \pm 0.1) \times 10^{0}[45 \%]$ & $(4.7 \pm 0.1) \times 10^{-1}[20 \%]$ & $(1.1 \pm 0.0) \times 10^{-1}[4 \%]$ \\
\hline & SED & $(2.0 \pm 0.9) \times 10^{-1}[8 \%]$ & $(5.7 \pm 2.5) \times 10^{-1}[24 \%]$ & $(1.1 \pm 0.4) \times 10^{0}[49 \%]$ & $(4.8 \pm 2.0) \times 10^{-1}[20 \%]$ & $(9.6 \pm 4.0) \times 10^{-2}[4 \%]$ \\
\hline \multirow[t]{3}{*}{70} & Stack & $(2.0 \pm 1.2) \times 10^{-1}[6 \%]$ & $(9.0 \pm 1.2) \times 10^{-1}[27 \%]$ & $(1.3 \pm 0.1) \times 10^{0}[38 \%]$ & $(7.1 \pm 0.6) \times 10^{-1}[21 \%]$ & $(1.7 \pm 0.2) \times 10^{-1}[5 \%]$ \\
\hline & $\mathrm{CC}$ & $(2.0 \pm 1.9) \times 10^{-1}[5 \%]$ & $(9.0 \pm 7.9) \times 10^{-1}[25 \%]$ & $(1.5 \pm 1.3) \times 10^{0}[41 \%]$ & $(7.2 \pm 0.7) \times 10^{-1}[20 \%]$ & $(1.7 \pm 0.2) \times 10^{-1}[4 \%]$ \\
\hline & SED & $(5.2 \pm 2.5) \times 10^{-1}[14 \%]$ & $(1.5 \pm 0.7) \times 10^{0}[43 \%]$ & $(3.0 \pm 1.2) \times 10^{0}[83 \%]$ & $(1.2 \pm 0.5) \times 10^{0}[32 \%]$ & $(2.3 \pm 0.9) \times 10^{-1}[6 \%]$ \\
\hline \multirow[t]{3}{*}{100} & Stack & $(8.8 \pm 0.8) \times 10^{-1}[10 \%]$ & $(2.2 \pm 0.1) \times 10^{0}[25 \%]$ & $(3.3 \pm 0.1) \times 10^{0}[37 \%]$ & $(1.8 \pm 0.1) \times 10^{0}[20 \%]$ & $(3.3 \pm 0.2) \times 10^{-1}[3 \%]$ \\
\hline & $\mathrm{CC}$ & $(9.7 \pm 1.2) \times 10^{-1}[8 \%]$ & $(2.4 \pm 0.4) \times 10^{0}[21 \%]$ & $(5.2 \pm 0.6) \times 10^{0}[46 \%]$ & $(1.9 \pm 0.1) \times 10^{0}[16 \%]$ & $(3.4 \pm 0.2) \times 10^{-1}[3 \%]$ \\
\hline & SED & $(7.4 \pm 3.6) \times 10^{-1}[6 \%]$ & $(2.2 \pm 1.0) \times 10^{0}[20 \%]$ & $(4.2 \pm 1.7) \times 10^{0}[38 \%]$ & $(1.6 \pm 0.7) \times 10^{0}[14 \%]$ & $(3.2 \pm 1.3) \times 10^{-1}[2 \%]$ \\
\hline \multirow[t]{3}{*}{160} & Stack & $(6.5 \pm 3.2) \times 10^{-1}[6 \%]$ & $(2.1 \pm 0.3) \times 10^{0}[21 \%]$ & $(3.6 \pm 0.3) \times 10^{0}[37 \%]$ & $(2.3 \pm 0.2) \times 10^{0}[24 \%]$ & $(5.1 \pm 0.5) \times 10^{-1}[5 \%]$ \\
\hline & $\mathrm{CC}$ & $(6.6 \pm 5.1) \times 10^{-1}[5 \%]$ & $(2.3 \pm 2.3) \times 10^{0}[18 \%]$ & $(6.4 \pm 2.9) \times 10^{0}[49 \%]$ & $(2.4 \pm 0.2) \times 10^{0}[18 \%]$ & $(5.2 \pm 0.5) \times 10^{-1}[4 \%]$ \\
\hline & SED & $(1.0 \pm 0.5) \times 10^{0}[7 \%]$ & $(3.1 \pm 1.4) \times 10^{0}[24 \%]$ & $(6.4 \pm 2.5) \times 10^{0}[50 \%]$ & $(2.5 \pm 1.1) \times 10^{0}[19 \%]$ & $(4.9 \pm 2.0) \times 10^{-1}[3 \%]$ \\
\hline \multirow[t]{3}{*}{250} & Stack & $(3.8 \pm 0.6) \times 10^{-1}[5 \%]$ & $(1.4 \pm 0.1) \times 10^{0}[20 \%]$ & $(2.5 \pm 0.1) \times 10^{0}[35 \%]$ & $(1.9 \pm 0.1) \times 10^{0}[27 \%]$ & $(5.3 \pm 0.2) \times 10^{-1}[7 \%]$ \\
\hline & $\mathrm{CC}$ & $(4.1 \pm 0.9) \times 10^{-1}[3 \%]$ & $(1.9 \pm 0.4) \times 10^{0}[16 \%]$ & $(6.1 \pm 0.6) \times 10^{0}[53 \%]$ & $(2.0 \pm 0.1) \times 10^{0}[17 \%]$ & $(5.3 \pm 0.2) \times 10^{-1}[4 \%]$ \\
\hline & SED & $(6.4 \pm 2.7) \times 10^{-1}[5 \%]$ & $(2.3 \pm 0.9) \times 10^{0}[19 \%]$ & $(6.4 \pm 2.9) \times 10^{0}[56 \%]$ & $(2.5 \pm 1.0) \times 10^{0}[21 \%]$ & $(6.2 \pm 2.6) \times 10^{-1}[5 \%]$ \\
\hline \multirow[t]{3}{*}{350} & Stack & $(1.8 \pm 0.4) \times 10^{-1}[4 \%]$ & $(8.0 \pm 0.6) \times 10^{-1}[18 \%]$ & $(1.5 \pm 0.1) \times 10^{0}[33 \%]$ & $(1.3 \pm 0.1) \times 10^{0}[29 \%]$ & $(4.0 \pm 0.1) \times 10^{-1}[9 \%]$ \\
\hline & $\mathrm{CC}$ & $(1.8 \pm 0.7) \times 10^{-1}[2 \%]$ & $(1.5 \pm 0.3) \times 10^{0}[16 \%]$ & $(4.9 \pm 0.5) \times 10^{0}[55 \%]$ & $(1.4 \pm 0.1) \times 10^{0}[15 \%]$ & $(4.1 \pm 0.1) \times 10^{-1}[4 \%]$ \\
\hline & SED & $(3.0 \pm 1.2) \times 10^{-1}[3 \%]$ & $(1.3 \pm 0.5) \times 10^{0}[14 \%]$ & $(4.7 \pm 2.6) \times 10^{0}[53 \%]$ & $(1.5 \pm 0.6) \times 10^{0}[17 \%]$ & $(4.6 \pm 1.9) \times 10^{-1}[5 \%]$ \\
\hline \multirow[t]{3}{*}{500} & Stack & $(6.3 \pm 2.8) \times 10^{-2}[3 \%]$ & $(2.9 \pm 0.3) \times 10^{-1}[15 \%]$ & $(5.9 \pm 0.3) \times 10^{-1}[31 \%]$ & $(5.6 \pm 0.2) \times 10^{-1}[30 \%]$ & $(2.0 \pm 0.1) \times 10^{-1}[10 \%]$ \\
\hline & $\mathrm{CC}$ & $(6.4 \pm 4.4) \times 10^{-2}[1 \%]$ & $(7.9 \pm 2.1) \times 10^{-1}[16 \%]$ & $(2.8 \pm 0.3) \times 10^{0}[58 \%]$ & $(6.3 \pm 0.3) \times 10^{-1}[13 \%]$ & $(2.1 \pm 0.1) \times 10^{-1}[4 \%]$ \\
\hline & SED & $(1.0 \pm 0.4) \times 10^{-1}[2 \%]$ & $(5.4 \pm 2.0) \times 10^{-1}[11 \%]$ & $(2.2 \pm 1.4) \times 10^{0}[46 \%]$ & $(6.1 \pm 2.4) \times 10^{-1}[12 \%]$ & $(2.1 \pm 0.9) \times 10^{-1}[4 \%]$ \\
\hline \multirow[t]{3}{*}{1100} & Stack & $(7.2 \pm 210.0) \times 10^{-5}[0 \%]$ & $(4.6 \pm 2.4) \times 10^{-3}[7 \%]$ & $(2.0 \pm 0.2) \times 10^{-2}[31 \%]$ & $(2.3 \pm 0.2) \times 10^{-2}[36 \%]$ & $(1.2 \pm 0.1) \times 10^{-2}[17 \%]$ \\
\hline & $\mathrm{CC}$ & $(7.2 \pm 340.0) \times 10^{-5}[0 \%]$ & $(6.5 \pm 17.0) \times 10^{-3}[3 \%]$ & $(1.2 \pm 0.3) \times 10^{-1}[68 \%]$ & $(2.7 \pm 0.2) \times 10^{-2}[15 \%]$ & $(1.2 \pm 0.1) \times 10^{-2}[6 \%]$ \\
\hline & SED & $(4.5 \pm 1.7) \times 10^{-3}[2 \%]$ & $(3.2 \pm 1.3) \times 10^{-2}[18 \%]$ & $(1.4 \pm 0.9) \times 10^{-1}[78 \%]$ & $(3.1 \pm 1.2) \times 10^{-2}[17 \%]$ & $(1.3 \pm 0.5) \times 10^{-2}[7 \%]$ \\
\hline
\end{tabular}

Notes. Stacked intensities in units of $\mathrm{nW} \mathrm{m} \mathrm{m}^{-2} \mathrm{sr}^{-1}$. Uncorrected intensities are labeled "Stack," completeness-corrected values are labeled "CC," and the best-fit SED values are labeled "SED." Blank spaces represent where the algorithm fails to adequately converge. Errors estimated with a Monte Carlo Simulation described in Section 3.4 .

Table 8

Stacked Intensities of Quiescent Galaxies in Bins of Stellar Mass

\begin{tabular}{|c|c|c|c|c|}
\hline $\begin{array}{l}\text { Band } \\
(\mu m)\end{array}$ & Type & $\begin{array}{c}\log \left(M / M_{\odot}\right)=9.0-10.0 \\
\left(\mathrm{nW} \mathrm{m}^{-2} \mathrm{sr}^{-1}\right)\end{array}$ & $\begin{array}{c}\log \left(M / M_{\odot}\right)=10.0-11.0 \\
\left(\mathrm{nW} \mathrm{m}^{-2} \mathrm{sr}^{-1}\right)\end{array}$ & $\begin{array}{c}\log \left(M / M_{\odot}\right)=11.0-12.0 \\
\left(\mathrm{nW} \mathrm{m}^{-2} \mathrm{sr}^{-1}\right)\end{array}$ \\
\hline \multirow[t]{3}{*}{24} & Stack & $(2.1 \pm 1.1) \times 10^{-3}[0 \%]$ & $(4.2 \pm 0.3) \times 10^{-2}[2 \%]$ & $(2.2 \pm 0.1) \times 10^{-2}[1 \%]$ \\
\hline & $\mathrm{CC}$ & $(2.1 \pm 1.6) \times 10^{-3}[0 \%]$ & $(5.1 \pm 0.4) \times 10^{-2}[2 \%]$ & $(2.2 \pm 0.1) \times 10^{-2}[0 \%]$ \\
\hline & SED & $(8.4 \pm 0.0) \times 10^{-3}[0 \%]$ & $(5.5 \pm 1.9) \times 10^{-2}[2 \%]$ & $(2.0 \pm 0.8) \times 10^{-2}[0 \%]$ \\
\hline \multirow[t]{3}{*}{70} & Stack & $(1.5 \pm 2.5) \times 10^{-2}[0 \%]$ & $(1.4 \pm 6.5) \times 10^{-2}[0 \%]$ & $(1.9 \pm 2.0) \times 10^{-2}[0 \%]$ \\
\hline & $\mathrm{CC}$ & $(1.8 \pm 4.4) \times 10^{-2}[0 \%]$ & $(3.7 \pm 11.0) \times 10^{-2}[1 \%]$ & $(1.9 \pm 2.0) \times 10^{-2}[0 \%]$ \\
\hline & SED & $(2.2 \pm 0.0) \times 10^{-2}[0 \%]$ & $(1.4 \pm 0.5) \times 10^{-1}[3 \%]$ & $(5.0 \pm 2.0) \times 10^{-2}[1 \%]$ \\
\hline \multirow[t]{3}{*}{100} & Stack & $(3.7 \pm 1.3) \times 10^{-2}[0 \%]$ & $(1.7 \pm 0.3) \times 10^{-1}[1 \%]$ & $(6.5 \pm 1.0) \times 10^{-2}[0 \%]$ \\
\hline & $\mathrm{CC}$ & $(3.8 \pm 2.3) \times 10^{-2}[0 \%]$ & $(2.2 \pm 0.5) \times 10^{-1}[1 \%]$ & $(6.6 \pm 1.0) \times 10^{-2}[0 \%]$ \\
\hline & SED & $(3.1 \pm 0.0) \times 10^{-2}[0 \%]$ & $(2.0 \pm 0.7) \times 10^{-1}[1 \%]$ & $(7.1 \pm 2.9) \times 10^{-2}[0 \%]$ \\
\hline \multirow[t]{3}{*}{160} & Stack & $(4.5 \pm 6.5) \times 10^{-2}[0 \%]$ & $(1.7 \pm 1.8) \times 10^{-1}[1 \%]$ & $(1.0 \pm 0.5) \times 10^{-1}[1 \%]$ \\
\hline & $\mathrm{CC}$ & $(2.1 \pm 1.2) \times 10^{-1}[1 \%]$ & $(2.0 \pm 2.9) \times 10^{-1}[1 \%]$ & $(1.0 \pm 0.5) \times 10^{-1}[0 \%]$ \\
\hline & SED & $(4.4 \pm 0.0) \times 10^{-2}[0 \%]$ & $(2.7 \pm 0.9) \times 10^{-1}[2 \%]$ & $(1.1 \pm 0.4) \times 10^{-1}[0 \%]$ \\
\hline \multirow[t]{3}{*}{250} & Stack & $(1.8 \pm 1.1) \times 10^{-2}[0 \%]$ & $(1.3 \pm 0.3) \times 10^{-1}[1 \%]$ & $(1.1 \pm 0.1) \times 10^{-1}[1 \%]$ \\
\hline & $\mathrm{CC}$ & $(2.1 \pm 1.9) \times 10^{-2}[0 \%]$ & $(3.2 \pm 0.5) \times 10^{-1}[2 \%]$ & $(1.1 \pm 0.1) \times 10^{-1}[0 \%]$ \\
\hline & SED & $(5.4 \pm 0.0) \times 10^{-2}[0 \%]$ & $(2.8 \pm 1.0) \times 10^{-1}[2 \%]$ & $(1.3 \pm 0.6) \times 10^{-1}[1 \%]$ \\
\hline \multirow[t]{3}{*}{350} & Stack & $(2.2 \pm 1.0) \times 10^{-2}[0 \%]$ & $(1.4 \pm 0.3) \times 10^{-1}[3 \%]$ & $(9.0 \pm 0.9) \times 10^{-2}[2 \%]$ \\
\hline & $\mathrm{CC}$ & $(5.8 \pm 1.7) \times 10^{-2}[0 \%]$ & $(3.2 \pm 0.5) \times 10^{-1}[3 \%]$ & $(9.0 \pm 0.9) \times 10^{-2}[1 \%]$ \\
\hline & SED & $(5.6 \pm 0.0) \times 10^{-2}[0 \%]$ & $(2.4 \pm 0.9) \times 10^{-1}[2 \%]$ & $(9.8 \pm 3.9) \times 10^{-2}[1 \%]$ \\
\hline \multirow[t]{3}{*}{500} & Stack & $(1.1 \pm 0.6) \times 10^{-2}[0 \%]$ & $(7.2 \pm 1.7) \times 10^{-2}[3 \%]$ & $(5.0 \pm 0.6) \times 10^{-2}[2 \%]$ \\
\hline & $\mathrm{CC}$ & $(4.1 \pm 1.2) \times 10^{-2}[0 \%]$ & $(1.6 \pm 0.3) \times 10^{-1}[3 \%]$ & $(5.0 \pm 0.6) \times 10^{-2}[1 \%]$ \\
\hline & SED & $(4.9 \pm 0.0) \times 10^{-2}[1 \%]$ & $(1.3 \pm 0.5) \times 10^{-1}[2 \%]$ & $(4.6 \pm 1.7) \times 10^{-2}[0 \%]$ \\
\hline \multirow[t]{3}{*}{1100} & Stack & $(4.6 \pm 5.1) \times 10^{-4}[0 \%]$ & $(2.2 \pm 1.3) \times 10^{-3}[3 \%]$ & $(2.4 \pm 0.5) \times 10^{-3}[3 \%]$ \\
\hline & $\mathrm{CC}$ & $(4.7 \pm 8.9) \times 10^{-4}[0 \%]$ & $(6.6 \pm 2.9) \times 10^{-3}[3 \%]$ & $(2.5 \pm 0.5) \times 10^{-3}[1 \%]$ \\
\hline & SED & $(7.5 \pm 0.0) \times 10^{-3}[4 \%]$ & $(9.1 \pm 3.3) \times 10^{-3}[5 \%]$ & $(3.1 \pm 1.1) \times 10^{-3}[1 \%]$ \\
\hline
\end{tabular}

Notes. Stacked intensities in units of $\mathrm{nW} \mathrm{m} \mathrm{m}^{-2} \mathrm{sr}^{-1}$. Uncorrected intensities are labeled "Stack," completeness-corrected values are labeled "CC," and the best-fit SED values are labeled "SED." Blank spaces represent where the algorithm fails to adequately converge. Errors estimated with a Monte Carlo Simulation described in Section 3.4. 


\section{REFERENCES}

Abadi, M. G., Moore, B., \& Bower, R. G. 1999, MNRAS, 308, 947 Addison, G. E., Dunkley, J., \& Bond, J. R. 2013, MNRAS, 436, 1674 Addison, G. E., Dunkley, J., Hajian, A., et al. 2012, ApJ, 752, 120 Alexander, D. M., Chary, R.-R., Pope, A., et al. 2008, ApJ, 687, 835 Alexander, D. M., Smail, I., Bauer, F. E., et al. 2005, Natur, 434, 738 Amblard, A., Cooray, A., Serra, P., et al. 2010, A\&A, 518, L9 Amblard, A., Cooray, A., Serra, P., et al. 2011, Natur, 470, 510 Assef, R. J., Kochanek, C. S., Brodwin, M., et al. 2010, ApJ, 713, 970 Austermann, J. E., Dunlop, J. S., Perera, T. A., et al. 2010, MNRAS, 401, 160 Barger, A. J., Cowie, L. L., Sanders, D. B., et al. 1998, Natur, 394, 248 Behroozi, P. S., Conroy, C., \& Wechsler, R. H. 2010, ApJ, 717, 379 Behroozi, P. S., Wechsler, R. H., \& Conroy, C. 2013a, ApJL, 762, L31 Behroozi, P. S., Wechsler, R. H., \& Conroy, C. 2013b, ApJ, 770, 57 Berta, S., Magnelli, B., Nordon, R., et al. 2011, A\&A, 532, A49 Bertin, E., \& Arnouts, S. 1996, A\&AS, 117, 393 Béthermin, M., Daddi, E., Magdis, G., et al. 2012a, ApJL, 757, L23 Béthermin, M., Dole, H., Beelen, A., \& Aussel, H. 2010, A\&A, 512, A78 Béthermin, M., Dole, H., Lagache, G., et al. 2011, A\&A, 529, A4 Béthermin, M., Doré, O., \& Lagache, G. 2012b, A\&A, 537, L5 Béthermin, M., Le Floc'h, E., Ilbert, O., et al. 2012c, A\&A, 542, A58 Blain, A. W., Barnard, V. E., \& Chapman, S. C. 2003, MNRAS, 338, 733 Blain, A. W., Chapman, S. C., Smail, I., \& Ivison, R. 2004, ApJ, 611, 725 Blain, A. W., Smail, I., Ivison, R. J., et al. 2002, PhR, 369, 111 Bourne, N., Dunne, L., Ivison, R. J., et al. 2011, MNRAS, 410, 1155 Bourne, N., Maddox, S. J., Dunne, L., et al. 2012, MNRAS, 421, 3027 Brammer, G. B., van Dokkum, P. G., \& Coppi, P. 2008, ApJ, 686, 1503 Brandl, B. R., Bernard-Salas, J., Spoon, H. W. W., et al. 2006, ApJ, 653, 1129 Bridge, C. R., Blain, A., Borys, C. J. K., et al. 2013, ApJ, 769, 91 Bruzual, G., \& Charlot, S. 2003, MNRAS, 344, 1000 Burgarella, D., Buat, V., Gruppioni, C., et al. 2013, A\&A, 554, A70 Caputi, K. I., Lagache, G., Yan, L., et al. 2007, ApJ, 660, 97 Casey, C. M. 2012, MNRAS, 425, 3094

Casey, C. M., Berta, S., Béthermin, M., et al. 2012, ApJ, 761, 140 Casey, C. M., Chapman, S. C., Beswick, R. J., et al. 2009, MNRAS, 399, 121 Chabrier, G. 2003, PASP, 115, 763

Chapin, E. L., Ade, P. A. R., Bock, J. J., et al. 2008, ApJ, 681, 428 Chapin, E. L., Pope, A., Scott, D., et al. 2009, MNRAS, 398, 1793 Chapman, S. C., Helou, G., Lewis, G. F., \& Dale, D. A. 2003a, ApJ, 588, 186 Chapman, S. C., Neri, R., Bertoldi, F., et al. 2008, ApJ, 689, 889 Chapman, S. C., Smail, I., Blain, A. W., \& Ivison, R. J. 2004, ApJ, 614, 671 Chapman, S. C., Windhorst, R., Odewahn, S., et al. 2003b, ApJ, 599, 92 Chary, R., \& Pope, A. 2010, arXiv:1003.1731

Cimatti, A., Daddi, E., \& Renzini, A. 2006, A\&A, 453, L29

Conselice, C. J., Bluck, A. F. L., Buitrago, F., et al. 2011, MNRAS, 413, 80 Cooray, A., \& Sheth, R. 2002, PhR, 372, 1

Daddi, E., Alexander, D. M., Dickinson, M., et al. 2007, ApJ, 670, 173

Dai, Y. S., Bergeron, J., Elvis, M., et al. 2012, ApJ, 753, 33

Dale, D. A., \& Helou, G. 2002, ApJ, 576, 159

Dale, D. A., Helou, G., Contursi, A., et al. 2001, ApJ, 549, 215

Davé, R., Finlator, K., Oppenheimer, B. D., et al. 2010, MNRAS, 404, 1355

De Bernardis, F., \& Cooray, A. 2012, ApJ, 760, 14

Devlin, M. J., Ade, P. A. R., Aretxaga, I., et al. 2009, Natur, 458, 737

Dey, A., Graham, J. R., Ivison, R. J., et al. 1999, ApJ, 519, 610

Dole, H., Lagache, G., Puget, J.-L., et al. 2006, A\&A, 451, 417

Dunne, L., Eales, S., Edmunds, M., et al. 2000, MNRAS, 315, 115

Dunne, L., \& Eales, S. A. 2001, MNRAS, 327, 697

Dunne, L., Gomez, H. L., da Cunha, E., et al. 2011, MNRAS, 417, 1510

Dye, S., Ade, P. A. R., Bock, J. J., et al. 2009, ApJ, 703, 285

Eales, S., Lilly, S., Webb, T., et al. 2000, AJ, 120, 2244

Elbaz, D., Dickinson, M., Hwang, H. S., et al. 2011, A\&A, 533, A119

Elbaz, D., Hwang, H. S., Magnelli, B., et al. 2010, A\&A, 518, L29

Engelbracht, C. W., Blaylock, M., Su, K. Y. L., et al. 2007, PASP, 119, 994

Ezawa, H., Kawabe, R., Kohno, K., \& Yamamoto, S. 2004, Proc. SPIE, 5489,763

Ezawa, H., Kohno, K., Kawabe, R., et al. 2008, Proc. SPIE, 7012, 701208 Farrah, D., Bernard-Salas, J., Spoon, H. W. W., et al. 2007, ApJ, 667, 149 Feltre, A., Hatziminaoglou, E., Hernán-Caballero, A., et al. 2013, MNRAS, 434, 2426

Fixsen, D. J., Dwek, E., Mather, J. C., et al. 1998, ApJ, 508, 123 Fontanot, F., De Lucia, G., Monaco, P., et al. 2009, MNRAS, 397, 1776 Fumagalli, M., Labbe, I., Patel, S. G., et al. 2013, arXiv:1308.4132 Furusawa, H., Kosugi, G., Akiyama, M., et al. 2008, ApJS, 176, 1 Glenn, J., Bock, J. J., Chattopadhyay, G., et al. 1998, Proc. SPIE, 3357, 326 Gordon, K. D., Engelbracht, C. W., Fadda, D., et al. 2007, PASP, 119, 1019 Griffin, M. J., Abergel, A., Abreu, A., et al. 2010, A\&A, 518, L3
Hall, N. R., Keisler, R., Knox, L., et al. 2010, ApJ, 718, 632

Hatziminaoglou, E., Omont, A., Stevens, J. A., et al. 2010, A\&A, 518, L33 Hauser, M. G., \& Dwek, E. 2001, ARA\&A, 39, 249

Hayward, C. C., Jonsson, P., Kerě̌, D., et al. 2012, MNRAS, 424, 951 Hayward, C. C., Narayanan, D., Kereš, D., et al. 2013, MNRAS, 428, 2529 Heinis, S., Buat, V., Béthermin, M., et al. 2013, MNRAS, 429, 1113 Hernquist, L. 1989, Natur, 340, 687

Hickox, R. C., Jones, C., Forman, W. R., et al. 2007, ApJ, 671, 1365 Hilton, M., Conselice, C. J., Roseboom, I. G., et al. 2012, MNRAS, 425, 540 Holder, G. P., Viero, M. P., Zahn, O., et al. 2013, ApJL, 771, L16 Holtzman, J. A., Hester, J. J., Casertano, S., et al. 1995, PASP, 107, 156 Hopkins, A. M., \& Beacom, J. F. 2006, ApJ, 651, 142

Hughes, D. H., Serjeant, S., Dunlop, J., et al. 1998, Natur, 394, 241 Hwang, H. S., Elbaz, D., Magdis, G., et al. 2010, MNRAS, 409, 75 Ilbert, O., McCracken, H. J., Le Fèvre, O., et al. 2013, A\&A, 556, A55 Jauzac, M., Dole, H., Le Floc'h, E., et al. 2011, A\&A, 525, A52 Juneau, S., Dickinson, M., Bournaud, F., et al. 2013, ApJ, 764, 176 Kennicutt, R. C., Jr. 1998, ARA\&A, 36, 189 Kirkpatrick, A., Pope, A., Alexander, D. M., et al. 2012, ApJ, 759, 139 Kirkpatrick, A., Pope, A., Charmandaris, V., et al. 2013, ApJ, 763, 123 Knapp, G. R., Gunn, J. E., \& Wynn-Williams, C. G. 1992, ApJ, 399, 76 Komatsu, E., Smith, K. M., Dunkley, J., et al. 2011, ApJS, 192, 18 Kovács, A., Omont, A., Beelen, A., et al. 2010, ApJ, 717, 29

Kriek, M., van Dokkum, P. G., Labbé, I., et al. 2009, ApJ, 700, 221 Kurczynski, P., \& Gawiser, E. 2010, AJ, 139, 1592

Labbé, I., Bouwens, R., Illingworth, G. D., \& Franx, M. 2006, ApJL, 649, L67 Labbé, I., Huang, J., Franx, M., et al. 2005, ApJL, 624, L81

Lacy, M., Storrie-Lombardi, L. J., Sajina, A., et al. 2004, ApJS, 154, 166 Lagache, G., Bavouzet, N., Fernandez-Conde, N., et al. 2007, ApJL, 665, L89 Lagache, G., Haffner, L. M., Reynolds, R. J., \& Tufte, S. L. 2000, A\&A, 354,247

Lagache, G., Puget, J.-L., \& Dole, H. 2005, ARA\&A, 43, 727

Lançon, A., \& Mouhcine, M. 2002, A\&A, 393, 167

Lawrence, A., Warren, S. J., Almaini, O., et al. 2007, MNRAS, 379, 1599

Le Floc'h, E., Papovich, C., Dole, H., et al. 2005, ApJ, 632, 169

Lee, N., Le Floc'h, E., Sanders, D. B., et al. 2010, ApJ, 717, 175

Levenson, L., Marsden, G., Zemcov, M., et al. 2010, MNRAS, 409, 83

Lonsdale, C. J., Smith, H. E., Rowan-Robinson, M., et al. 2003, PASP, 115, 897

Lutz, D., Poglitsch, A., Altieri, B., et al. 2011, A\&A, 532, A90

Magdis, G. E., Elbaz, D., Hwang, H. S., et al. 2010, MNRAS, 409, 22

Magnelli, B., Lutz, D., Berta, S., et al. 2010, A\&A, 518, L28

Magnelli, B., Lutz, D., Santini, P., et al. 2012, A\&A, 539, A155

Magnelli, B., Popesso, P., Berta, S., et al. 2013, A\&A, 553, A132

Marchesini, D., Whitaker, K. E., Brammer, G., et al. 2010, ApJ, 725, 1277

Marsden, G., Ade, P. A. R., Bock, J. J., et al. 2009, ApJ, 707, 1729

McCarthy, I. G., Frenk, C. S., Font, A. S., et al. 2008, MNRAS, 383, 593

Menéndez-Delmestre, K., Blain, A. W., Smail, I., et al. 2009, ApJ, 699, 667

Michałowski, M. J., Murphy, E. J., Hjorth, J., et al. 2010a, A\&A, 522, A15

Michałowski, M. J., Watson, D., \& Hjorth, J. 2010b, ApJ, 712, 942

Miville-Deschênes, M.-A., Lagache, G., \& Puget, J.-L. 2002, A\&A, 393, 749

Mo, H. J., \& White, S. D. M. 1996, MNRAS, 282, 347

Moncelsi, L., Ade, P. A. R., Chapin, E. L., et al. 2011, ApJ, 727, 83

Moster, B. P., Somerville, R. S., Maulbetsch, C., et al. 2010, ApJ, 710, 903

Mullaney, J. R., Alexander, D. M., Goulding, A. D., \& Hickox, R. C. 2011, MNRAS, 414, 1082

Murphy, E. J., Chary, R.-R., Dickinson, M., et al. 2011, ApJ, 732, 126 Muzzin, A., Marchesini, D., Stefanon, M., et al. 2013a, ApJ, 777, 18 Muzzin, A., Marchesini, D., Stefanon, M., et al. 2013b, ApJS, 206, 8 Nguyen, H. T., Schulz, B., Levenson, L., et al. 2010, A\&A, 518, L5 Noeske, K. G., Weiner, B. J., Faber, S. M., et al. 2007, ApJL, 660, L43 Oliver, S., Frost, M., Farrah, D., et al. 2010a, MNRAS, 405, 2279 Oliver, S. J., Bock, J., Altieri, B., et al. 2012, MNRAS, 424, 1614 Oliver, S. J., Wang, L., Smith, A. J., et al. 2010b, A\&A, 518, L21

Ott, S. 2010, in ASP Conf. Ser. 434, Astronomical Data Analysis Software and Systems XIX, ed. Y. Mizumoto, K.-I. Morita, \& M. Ohishi (San Francisco, CA: ASP), 139

Papovich, C., Dole, H., Egami, E., et al. 2004, ApJS, 154, 70 Pascale, E., Ade, P. A. R., Bock, J. J., et al. 2008, ApJ, 681, 400 Pascale, E., Ade, P. A. R., Bock, J. J., et al. 2009, ApJ, 707, 1740 Peacock, J. A., \& Smith, R. E. 2000, MNRAS, 318, 1144

Penner, K., Pope, A., Chapin, E. L., et al. 2011, MNRAS, 410, 2749

Pérez-González, P. G., Rieke, G. H., Villar, V., et al. 2008a, ApJ, 675, 234

Pérez-González, P. G., Trujillo, I., Barro, G., et al. 2008b, ApJ, 687, 50

Piazzo, L. 2013, arXiv:1301.1246

Piovan, L., Tantalo, R., \& Chiosi, C. 2003, A\&A, 408, 559

Planck Collaboration, Ade, P. A. R., Aghanim, N., et al. 2011, A\&A, 536, A18 
Planck Collaboration, Ade, P. A. R., Aghanim, N., et al. 2013, arXiv:1309.0382 Poglitsch, A., Waelkens, C., Geis, N., et al. 2010, A\&A, 518, L2 Polletta, M., Weedman, D., Hönig, S., et al. 2008, ApJ, 675, 960

Polletta, M. d. C., Wilkes, B. J., Siana, B., et al. 2006, ApJ, 642, 673

Pope, A., Chary, R.-R., Alexander, D. M., et al. 2008, ApJ, 675, 1171

Puget, J.-L., Abergel, A., Bernard, J.-P., et al. 1996, A\&A, 308, L5

Quadri, R. F., \& Williams, R. J. 2010, ApJ, 725, 794

Quadri, R. F., Williams, R. J., Franx, M., \& Hildebrandt, H. 2012, ApJ, 744, 88

Rawle, T. D., Rex, M., Egami, E., et al. 2012, ApJ, 756, 106

Richards, G. T., Lacy, M., Storrie-Lombardi, L. J., et al. 2006, ApJS, 166, 470

Rieke, G. H., Young, E. T., Engelbracht, C. W., et al. 2004, ApJS, 154, 25

Rodighiero, G., Cimatti, A., Gruppioni, C., et al. 2010, A\&A, 518, L25

Roehlly, Y., Buat, V., Heinis, S., et al. 2011, in ASP Conf. Ser. 442, Astronomical

Data Analysis Software and Systems XX, ed. I. N. Evans, A. Accomazzi,

D. J. Mink, \& A. H. Rots (San Francisco, CA: ASP), 25

Roseboom, I. G., Ivison, R. J., Greve, T. R., et al. 2012, MNRAS, 419, 2758

Roseboom, I. G., Oliver, S. J., Kunz, M., et al. 2010, MNRAS, 409, 48

Rowan-Robinson, M., \& Crawford, J. 1989, MNRAS, 238, 523

Salim, S., Dickinson, M., Michael Rich, R., et al. 2009, ApJ, 700, 161

Santini, P., Maiolino, R., Magnelli, B., et al. 2010, A\&A, 518, L154

Serjeant, S., Bertoldi, F., Blain, A. W., et al. 2010, A\&A, 518, L7

Serjeant, S., Mortier, A. M. J., Ivison, R. J., et al. 2004, ApJS, 154, 118

Shang, C., Haiman, Z., Knox, L., \& Oh, S. P. 2012, MNRAS, 421, 2832

Skibba, R. A., Engelbracht, C. W., Dale, D., et al. 2011, ApJ, 738, 89
Smail, I., Ivison, R. J., \& Blain, A. W. 1997, ApJL, 490, L5

Smail, I., Ivison, R. J., Blain, A. W., \& Kneib, J.-P. 2002, MNRAS, 331, 495

Stansberry, J. A., Gordon, K. D., Bhattacharya, B., et al. 2007, PASP, 119, 1038

Stern, D., Assef, R. J., Benford, D. J., et al. 2012, ApJ, 753, 30

Surace, J. A. 2005, Technical Report, The SWIRE Data release 2 yCat, 2302, 0

Swinyard, B. M., Ade, P., Baluteau, J.-P., et al. 2010, A\&A, 518, L4

Symeonidis, M., Vaccari, M., Berta, S., et al. 2013, MNRAS, 431, 2317

Traficante, A., Calzoletti, L., Veneziani, M., et al. 2011, MNRAS, 416, 2932

Valiante, E., Lutz, D., Sturm, E., et al. 2009, ApJ, 701, 1814

Viero, M. P., Ade, P. A. R., Bock, J. J., et al. 2009, ApJ, 707, 1766

Viero, M. P., Asboth, V., Roseboom, I. G., et al. 2013a, arXiv:1308.4389

Viero, M. P., Moncelsi, L., Mentuch, E., et al. 2012, MNRAS, 421, 2161

Viero, M. P., Wang, L., Zemcov, M., et al. 2013b, ApJ, 772, 77

Wang, L., Farrah, D., Oliver, S. J., et al. 2013, MNRAS, 431, 648

Warren, S. J., Hambly, N. C., Dye, S., et al. 2007, MNRAS, 375, 213

Weiß, A., Kovács, A., Coppin, K., et al. 2009, ApJ, 707, 1201

Wiebe, D. V., Ade, P. A. R., Bock, J. J., et al. 2009, ApJ, 707, 1809

Wieprecht, E., Schreiber, J., de Jong, J., et al. 2009, in ASP Conf. Ser. 411, Astronomical Data Analysis Software and Systems XVIII, ed. D. A Bohlender, D. Durand, \& P. Dowler (San Francisco, CA: ASP), 531

Williams, R. J., Quadri, R. F., Franx, M., et al. 2009, ApJ, 691, 1879

Wilson, G. W., Austermann, J. E., Perera, T. A., et al. 2008, MNRAS, 386, 807

Wuyts, S., Labbé, I., Franx, M., et al. 2007, ApJ, 655, 51 